\title{
One-component cationic photoinitiators from tunable benzylidene scaffolds for 3D printing applications
}

Filip Petko ${ }^{1}$, Mariusz Galek$^{1}$, Emilia Hola ${ }^{2}$, Roman Popielarz ${ }^{2}$, Joanna Orty ${ }^{* 1,2}$

1 Photo HiTech Ltd., Bobrzyńskiego 14, 30-348 Cracow, Poland

${ }^{2}$ Cracow University of Technology, Faculty of Chemical Engineering and Technology, Warszawska 24, 31-155 Cracow, Poland

*Correspondence: jortyl@pk.edu.pl and joanna.ortyl@photohitech.com 


\section{Contents}

\section{Section A. Materials and methods}

\section{Section B. Synthetic procedures}

Section C. Photochemical experiments

\section{Section A. Materials and methods}

All reagents were purchased from Sigma-Aldrich, Alfa Aesar, Acros Organics and Fluorochem and used without further purification. Anhydrous THF was obtained from sodium/benzophenone ketyl distillation. Silica TLC plates with F-254 nm indicator were purchased from Silicycle Inc.Flash chromatography was performed on Puriflash XS 420, Interchim.Melting points were determined on a Stuart SMP30 apparatus and are uncorrected.

${ }^{1} \mathrm{H}$ NMR spectra were recorded on Avance III HD $400 \mathrm{MHz}$ Bruker, where chemical shifts were determined with a residual proton of the solvent as standard.

Structures of monomers used during polymerization tests are shown on figure S1.

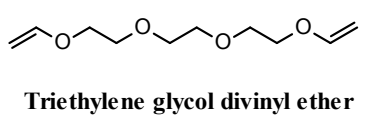

(TEGDVE)

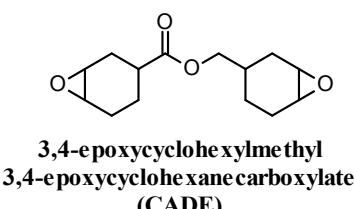

(CADE)

Figure S1. Structures of monomers used during photopolymerization experiments.

\section{Section B. Synthetic procedures}

\section{Synthesis of 2-[(2-methoxyphenyl)methylene]propanedinitrile derivatives}

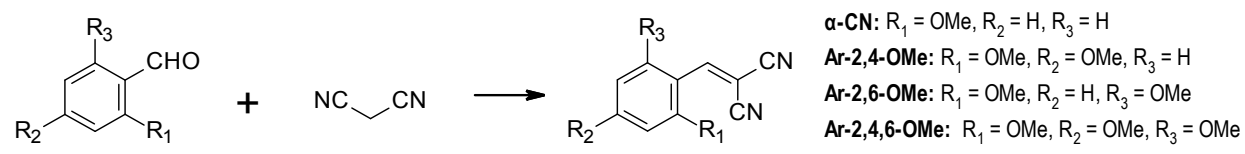

O-anisaldehyde or other benzaldehyde derivatives $(13.60 \mathrm{~g}, 0,10 \mathrm{~mol})$ and malononitrile $(6.02 \mathrm{~g}, 0.10 \mathrm{~mol})$ were dissolved in $\mathrm{MeOH}(50 \mathrm{ml})$, piperidine $(0,85 \mathrm{~g}, 0.99 \mathrm{ml}, 0.01 \mathrm{~mol})$ was added and the reaction mixture was stirred $4 \mathrm{~h}$ at RT. Then volatiles were removed under vacuum and the residue was crystalized from methanol $(16.93 \mathrm{~g}$, $92 \%)$.

In the similar way below listed compounds were obtained:

\begin{tabular}{|c|c|c|}
\hline Compound & Spectroscopic properties & Yield: \\
\hline$\alpha-C N$ & $\begin{array}{l}\text { 2-[(2-methoxyphenyl)methylene]propanedinitrile } \\
\mathrm{mp}=83-85^{\circ} \mathrm{C} \\
\text { lit. } \mathrm{mp}=84-85^{\circ} \mathrm{C}^{1} \\
{ }^{1} \mathrm{H} \text { NMR }(400 \mathrm{MHz}, \mathrm{DMSO}) \delta 8.47(\mathrm{~s}, 1 \mathrm{H}), 7.96(\mathrm{dd}, \mathrm{J}=7.9,1.5 \mathrm{~Hz}, 1 \mathrm{H}), 7.70 .766(\mathrm{~m} \text {, } \\
\text { 1H), } 7.24(\mathrm{~d}, \mathrm{~J}=8.5 \mathrm{~Hz}, 1 \mathrm{H}), 7.15(\mathrm{t}, \mathrm{J}=7.7 \mathrm{~Hz}, 1 \mathrm{H}), 3.91(\mathrm{~s}, 3 \mathrm{H})\end{array}$ & $92 \%$ \\
\hline
\end{tabular}




\begin{tabular}{|c|c|c|}
\hline$A r-2,4-O M e$ & $\begin{array}{l}\text { 2-[(2,4-dimethoxyphenyl)methylene]propanedinitrile } \\
\mathrm{mp}=141-143^{\circ} \mathrm{C} \\
\text { lit. } \mathrm{mp}=140-142^{\circ} \mathrm{C}^{2} \\
{ }^{1} \mathrm{H} \mathrm{NMR}(400 \mathrm{MHz}, \mathrm{DMSO}) \delta 8.24(\mathrm{~s}, 1 \mathrm{H}), 7.59(\mathrm{t}, \mathrm{J}=8.5 \mathrm{~Hz}, 1 \mathrm{H}), 6.80(\mathrm{~d}, \mathrm{~J}=8.5 \mathrm{~Hz} \text {, } \\
2 \mathrm{H}), 3.88(\mathrm{~s}, 6 \mathrm{H})\end{array}$ & $92 \%$ \\
\hline $2,6-O M e$ & $\begin{array}{l}\text { 2-[(2,6-dimethoxyphenyl)methylene]propanedinitrile } \\
\mathrm{mp}=150^{\circ} \mathrm{C} \\
\text { lit. } \mathrm{mp}=148^{\circ} \mathrm{C}^{3} \\
{ }^{1} \mathrm{H} \mathrm{NMR}(400 \mathrm{MHz}, \mathrm{DMSO}) \delta 8.24(\mathrm{~s}, 1 \mathrm{H}), 7.59(\mathrm{t}, \mathrm{J}=8.4 \mathrm{~Hz}, 1 \mathrm{H}), 6.80(\mathrm{~d}, \mathrm{~J}=8.4 \mathrm{~Hz} \text {, } \\
2 \mathrm{H}), 3.88(\mathrm{~s}, 6 \mathrm{H})\end{array}$ & $90 \%$ \\
\hline Ar-2,4,6-OMe & $\begin{array}{l}\text { 2-[(2,4,6-trimethoxyphenyl)methylene]propanedinitrile } \\
\mathrm{mp}=171-173^{\circ} \mathrm{C} \\
\text { lit. } \mathrm{mp}=175^{\circ} \mathrm{C}^{4} \\
{ }^{1} \mathrm{H} \mathrm{NMR}(400 \mathrm{MHz}, \mathrm{DMSO}) \delta 7.98(\mathrm{~s}, 1 \mathrm{H}), 6.35(\mathrm{~s}, 2 \mathrm{H}), 3.91(\mathrm{~s}, 3 \mathrm{H}), 3.89(\mathrm{~s}, 6 \mathrm{H})\end{array}$ & $78 \%$ \\
\hline
\end{tabular}

2. Synthesis of 3-(2-methoxyphenyl)-2-methylsulfonyl-prop-2-enenitrile

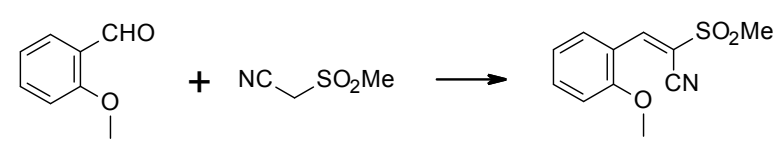

O-anisaldehyde $(13.60 \mathrm{~g}, 0,10 \mathrm{~mol})$ and methylsulfonylacetonitrile $(11.90 \mathrm{~g}, 0.10 \mathrm{~mol})$ were dissolved in $\mathrm{MeOH}$ $(100 \mathrm{ml})$, piperidine $(0,85 \mathrm{~g}, 0.99 \mathrm{ml}, 0.01 \mathrm{~mol})$ was added and the reaction mixture was stirred $20 \mathrm{~h}$ at RT. An excess of water was added, resulted mixture was extracted with EtOAc, combined organic layers were washed with water and brine, dried over anhydrous $\mathrm{Na}_{2} \mathrm{SO}_{4}$, concentrated under vacuum and submitted on flash chromatography $\left(\mathrm{SiO}_{2}\right.$, hexane/EtOAc 7:3) afforded $(22.75 \mathrm{~g}, 96 \%)$.

\begin{tabular}{|c|c|c|}
\hline Compound & Spectroscopic properties & Yield: \\
\hline$\alpha-\mathrm{SO}_{2} \mathrm{Me}$ & 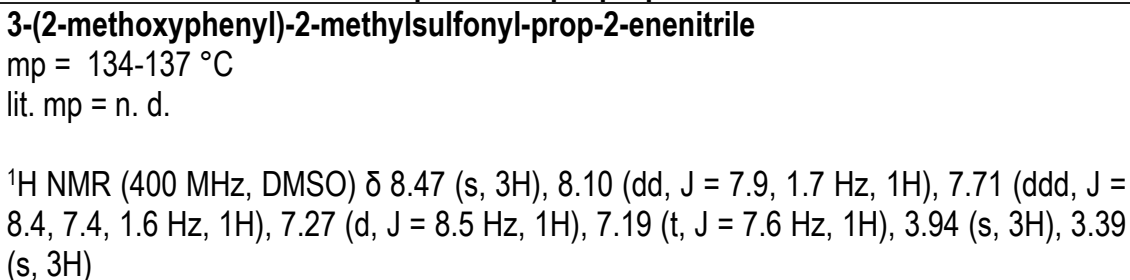 & $96 \%$ \\
\hline
\end{tabular}

\section{Synthesis of methyl (E)-2-cyano-3-(2-methoxyphenyl)prop-2-enoate}

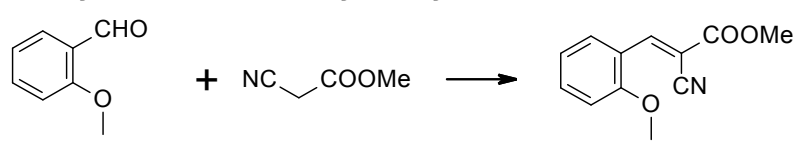

O-anisaldehyde $(13.60 \mathrm{~g}, 0,10 \mathrm{~mol})$ and ethyl cyanoacetate $(11.30 \mathrm{~g}, 10.63 \mathrm{ml}, 0.10 \mathrm{~mol})$ were dissolved in $\mathrm{MeOH}(130 \mathrm{ml})$, piperidine $(0,85 \mathrm{~g}, 0.99 \mathrm{ml}, 0.01 \mathrm{~mol})$ was added and the reaction mixture was stirred $20 \mathrm{~h}$ at $\mathrm{RT}$. The reaction mixture was cooled to $-20^{\circ} \mathrm{C}$ and resulted solid was filtered off, washed with cold methanol and dried afforded pure product as a methyl ester $(12.59 \mathrm{~g}, 58 \%)$. 


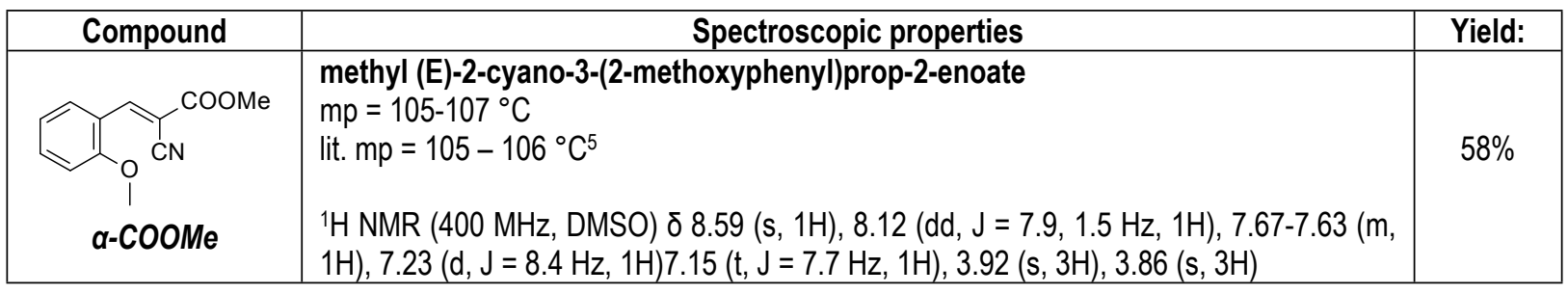

\section{Synthesis of diethyl 2-[(2-methoxyphenyl)methylene]propanedioate}

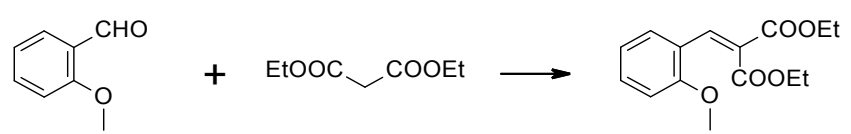

O-anisaldehyde $(6.80,0,05 \mathrm{~mol})$ and diethyl malonate $(8.00 \mathrm{~g}, 7.55 \mathrm{ml}, 0.05 \mathrm{~mol})$ were dissolved in pyridine (25 $\mathrm{ml})$, piperidine $(4.26 \mathrm{~g}, 4.94 \mathrm{ml}, 0.05 \mathrm{~mol})$ was added and the reaction mixture was stirred $3 \mathrm{~h}$ at $80^{\circ} \mathrm{C}$. Water $(50$ $\mathrm{ml}$ ) was added to the cooled reaction mixture and resulted solution was extracted with EtOAc, combined organic layers were washed with $5 \%$ aq $\mathrm{HCl}$, then with aq $\mathrm{NaHCO}_{3}$ and brine, dried over anhydrous $\mathrm{Na}_{2} \mathrm{SO}_{4}$, concentrated under vacuum and submitted on column chromatography $\left(\mathrm{SiO}_{2}\right.$, hexane/EtOAc 9:1) afforded colorless oil (11.12 g, 80\%).

\begin{tabular}{|c|c|c|}
\hline Compound & Spectroscopic properties & Yield: \\
\hline$\alpha-2-C O O E t$ & $\begin{array}{l}\text { diethyl 2-[(2-methoxyphenyl)methylene]propanedioate } \\
\text { mp = n. d. } \\
\text { lit. mp }=51-52^{\circ} \mathrm{C}^{6} \\
\\
{ }^{1} \mathrm{H} \mathrm{NMR}(400 \mathrm{MHz}, \mathrm{DMSO}) \delta 7.90(\mathrm{~s}, 1 \mathrm{H}), 7.49-7.45(\mathrm{~m}, 1 \mathrm{H}), 7.31(\mathrm{dd}, \mathrm{J}=7.8,1.5 \mathrm{~Hz} \text {, } \\
1 \mathrm{H}), 7.12(\mathrm{~d}, \mathrm{~J}=8.4 \mathrm{~Hz}, 1 \mathrm{H}), 7.00(\mathrm{t}, \mathrm{J}=7.7 \mathrm{~Hz}, 1 \mathrm{H}), 4.23(\text { quint, J }=7.0 \mathrm{~Hz}, 4 \mathrm{H}), 3.85 \\
(\mathrm{~s}, 3 \mathrm{H}), 1.25(\mathrm{t}, \mathrm{J}=7.3 \mathrm{~Hz}, 3 \mathrm{H}), 1.17(\mathrm{t}, \mathrm{J}=7.1 \mathrm{~Hz}, 3 \mathrm{H})\end{array}$ & $80 \%$ \\
\hline
\end{tabular}

\section{Synthesis of 2-[1-(2-methoxyphenyl)ethylidene]propanedinitrile}

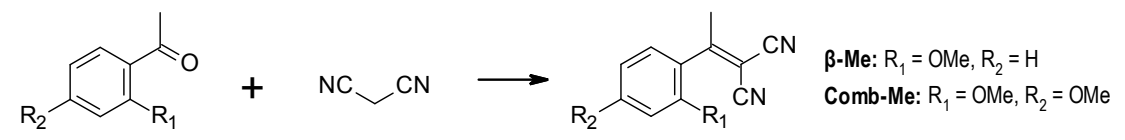

2'-metoxyacetophenone or 2',4'-dimetoxyacetophenone (15.01 g, $13.77 \mathrm{ml}, 0.10 \mathrm{~mol})$, malononitrile $(6.60 \mathrm{~g}, 0.1$ $\mathrm{mol})$ and $\mathrm{NH}_{4} \mathrm{OAc}(3.85 \mathrm{~g}, 0.05 \mathrm{~mol})$ were mixed with toluene $(150 \mathrm{ml})$ and $\mathrm{HOAc}(30 \mathrm{ml})$, then refluxed under Dean-Stark apparatus for $2 \mathrm{~h}$. Volatiles were removed under vacuum, the residue was taken up with EtOAc, washed with aq $\mathrm{NaHCO}_{3}$ and brine, dried over anhydrous $\mathrm{Na}_{2} \mathrm{SO}_{4}$, filtrated and concentrated, the residue was crystalized from methanol $(18.03 \mathrm{~g}, 91 \%)$.

In the similar way below compound was obtained:

\begin{tabular}{|c|c|c|}
\hline Compound & Spectroscopic properties & Yield: \\
\hline$\beta-M e$ & $\begin{array}{l}\text { 2-[1-(2-methoxyphenyl)ethylidene]propanedinitrile } \\
\mathrm{mp}=66-69^{\circ} \mathrm{C} \\
\text { lit. } \mathrm{mp}=139-145^{\circ} \mathrm{C}^{7} \\
{ }^{1} \mathrm{H} \mathrm{NMR}(400 \mathrm{MHz}, \mathrm{DMSO}) \delta 7.55-7.51(\mathrm{~m}, 1 \mathrm{H}), 7.39(\mathrm{dd}, \mathrm{J}=7.6,1.7 \mathrm{~Hz}, 1 \mathrm{H}), 7.20(\mathrm{~d} \text {, } \\
\mathrm{J}=8.3 \mathrm{~Hz}, 1 \mathrm{H}), 7.09(\mathrm{td}, \mathrm{J}=7.3,0.8 \mathrm{~Hz}, 1 \mathrm{H}), 3.85(\mathrm{~s}, 3 \mathrm{H}), 2.56(\mathrm{~s}, 3 \mathrm{H})\end{array}$ & $91 \%$ \\
\hline Comb-Me & $\begin{array}{l}\text { 2-[1-(2,4-dimethoxyphenyl)ethylidene]propanedinitrile } \\
\mathrm{mp}=108-110^{\circ} \mathrm{C} \\
\text { lit. } \mathrm{mp}=\mathrm{n} . \mathrm{d} . \\
\begin{array}{l}{ }^{1} \mathrm{H} \mathrm{NMR}(400 \mathrm{MHz}, \mathrm{DMSO}) \delta 8.48(\mathrm{~s}, 1 \mathrm{H}), 8.11-8.07(\mathrm{~m}, 2 \mathrm{H}), 7.66(\mathrm{tt}, \mathrm{J}=7.5,1.4 \mathrm{~Hz} \text {, } \\
1 \mathrm{H}), 7.51(\mathrm{tt}, \mathrm{J}=7.5,1.4 \mathrm{~Hz}, 2 \mathrm{H}), 6.98(\mathrm{~s}, 1 \mathrm{H}), 4.07(\mathrm{~s}, 3 \mathrm{H}), 3.98(\mathrm{~s}, 3 \mathrm{H}), 2.55(\mathrm{~s}, 3 \mathrm{H})\end{array}\end{array}$ & $84 \%$ \\
\hline
\end{tabular}




\section{Synthesis of 2-[(2-methoxyphenyl)-phenyl-methylene]propanedinitrile and 2-[(2,4-dimethoxyphenyl)-}

phenyl-methylene]propanedinitrile

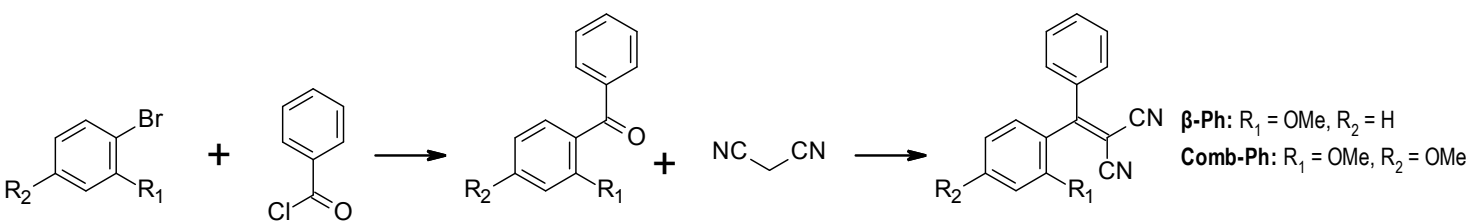

Magnesium $(0.76 \mathrm{~g}, 0.031 \mathrm{~mol})$ in anhydrous THF $(50 \mathrm{ml})$ was treated with DIBALH $(1 \mathrm{M}, 0.50 \mathrm{ml})$ and stirred at $\mathrm{RT}$ for $5 \mathrm{~min}$. Then 2-bromoanisole or 2,4-bromoanisole $(4.65 \mathrm{~g}, 3.10 \mathrm{ml}, 0.025 \mathrm{~mol})$ was added. When formation of Gringard reagent ceased CuCNx2LiCl in THF solution was added $(1 \mathrm{M}, 25.00 \mathrm{ml})$ and the mixture was further stirred for $15 \mathrm{~min}$. Then benzoyl chloride $(3.67 \mathrm{~g}, 3.03 \mathrm{ml}, 0.026 \mathrm{~mol})$ was added and the reaction mixture was further stirred for $1 \mathrm{~h}$. The reaction was quenched by addition of aq $\mathrm{NH}_{4} \mathrm{Cl}$, filtrated through Celite pad, diluted with water $(100 \mathrm{ml})$ and the resulted mixture was extracted with EtOAc, organic layers were combined, washed with brine, dried over anhydrous $\mathrm{Na}_{2} \mathrm{SO}_{4}$, concentrated and submitted on flash chromatography $\left(\mathrm{SiO}_{2}\right.$, hex/EtOAc 4:1) afforded white solid $(3.71 \mathrm{~g}, 70 \%)$ which was directly used in the next step.

Product was the previous step $(3.70 \mathrm{~g}, 0.017 \mathrm{~mol})$ and malononitrile $(2.88 \mathrm{~g}, 0.042 \mathrm{~mol})$ were dissolved in DCM $(110 \mathrm{ml})$ and slowly treated with $\mathrm{TiCl}_{4}(16.12 \mathrm{~g}, 9.32 \mathrm{ml}, 0.085 \mathrm{~mol})$ and pyridine $(32 \mathrm{ml})$, then the mixture was stirred overnight at RT. 10\% hydrochloric acid $(180 \mathrm{ml})$ was added and the resulted mixture was extracted with EtOAc, combined organic layers were washed with water, aq $\mathrm{NaHCO}_{3}$ and brine, dried over anhydrous $\mathrm{Na}_{2} \mathrm{SO}_{4}$, concentrated under vacuum and submitted on flash chromatography $\left(\mathrm{SiO}_{2}\right.$, hex/EtOAc 4:1) afforded white solid (3.85 g, 87\%).

In similar way below compound was obtained:

\begin{tabular}{|c|c|c|}
\hline Compound & Spectroscopic properties & Yield: \\
\hline$\beta-P h$ & 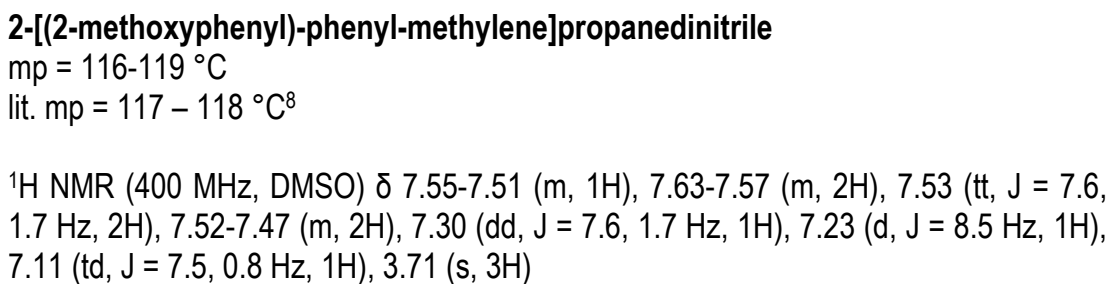 & $87 \%$ \\
\hline Comb-Ph & 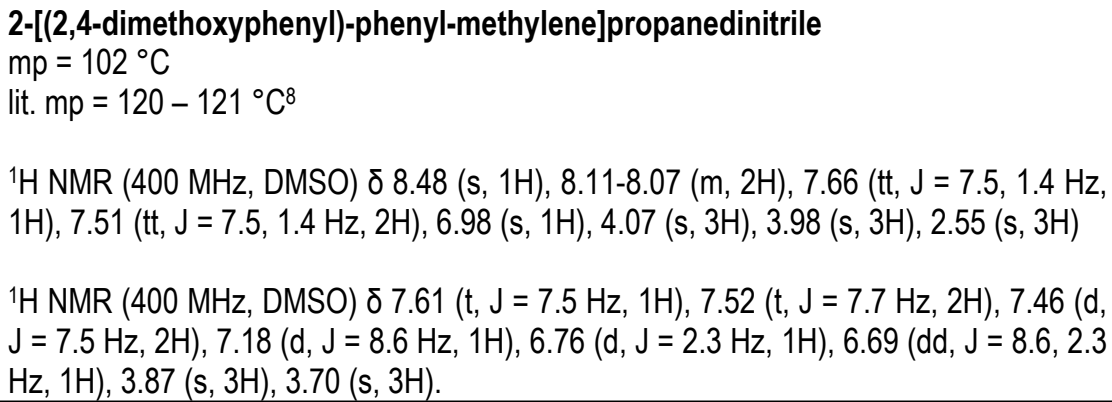 & $68 \%$ \\
\hline
\end{tabular}

7. Synthesis of 2-[methoxy-(2-methoxyphenyl)methylene]propanedinitrile and 2-[(2,4-dimethoxyphenyl)methoxy-methylene]propanedinitrile

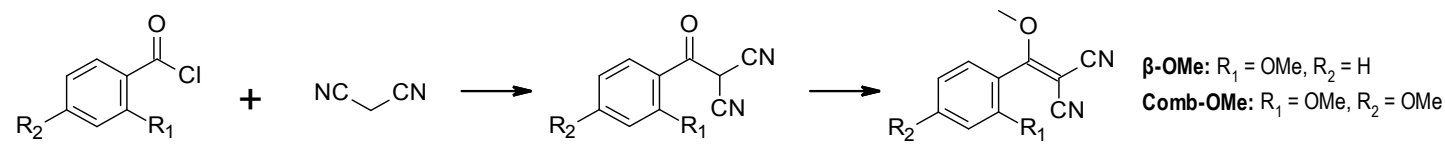


To a suspension of $\mathrm{NaH}(60 \%, 2.00 \mathrm{~g}, 0.050 \mathrm{~mol})$ in THF $(10 \mathrm{ml})$ at $0{ }^{\circ} \mathrm{C}$ a solution of malononitrile $(1.65 \mathrm{~g}, 0.025$ $\mathrm{mol})$ in THF $(10 \mathrm{ml})$ was added and the resulted mixture was stirred for $20 \mathrm{~min}$. Then a solution of 2metoxybenzoyl chloride or 2,4-metoxybenzoyl chloride $(4.25 \mathrm{~g}, 0.025 \mathrm{~mol})$ in THF $(10 \mathrm{ml})$ was added the reaction mixture was stirred overnight at RT. $5 \%$ hydrochloric acid was added to make reaction mixture acidic, then extraction was perform with usage of EtOAc, organic layers were combined, washed with aq $\mathrm{NaHCO}_{3}$ and brine, dried over anhydrous $\mathrm{Na}_{2} \mathrm{SO}_{4}$, concentrated to dryness and directly used in the next step.

To a solution of product from previous step $(4.75 \mathrm{~g}, 0.024 \mathrm{~mol})$ in 1,4-dioxane $(20 \mathrm{ml})$ and water $(3.50 \mathrm{ml})$ $\mathrm{NaHCO}_{3}(16.13 \mathrm{~g}, 0.192 \mathrm{~mol})$ was added. After $5 \mathrm{~min}$ - of stirring at RT dimethyl sulfate $(21.19 \mathrm{~g}, 15.90 \mathrm{ml}, 0.168$ mol) was added and the mixture was refluxed for $2 \mathrm{~h}$. Water $(100 \mathrm{ml})$ was added and the mixture was extracted with EtOAc, combined organic layers were washed with brine, dried over anhydrous $\mathrm{Na}_{2} \mathrm{SO}_{4}$, concentrated under vacuum and purified on flash chromatography $\left(\mathrm{SiO}_{2}\right.$, hex/EtOAc 1:1) afforded white solid $(3.65 \mathrm{~g}, 71 \%)$.

In similar way below compound was obtained:

\begin{tabular}{|l|l|c|}
\hline Compound & \multicolumn{1}{|c|}{ Spectroscopic properties } & \multicolumn{1}{|c|}{ Yield: } \\
\hline
\end{tabular}

8. Synthesis of 2-(2-methoxyphenyl)ethene-1,1,2-tricarbonitrile and 2-(2,4-dimethoxyphenyl)ethene-1,1,2tricarbonitrile

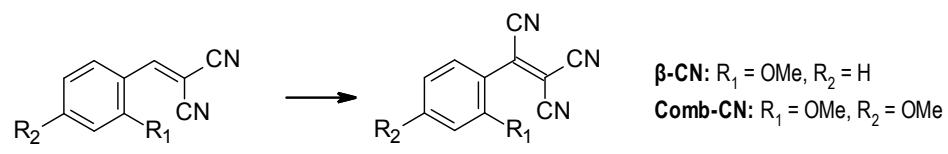

2-[(2-methoxyphenyl)methylene]propanedinitrile or 2-[(2,4-dimethoxyphenyl)methylene]propanedinitrile $(0.92$ $\mathrm{g}, 0.005 \mathrm{~mol})$ and $\mathrm{KCN}(0.33 \mathrm{~g}, 0.005 \mathrm{~mol})$ were dissolved in a mixture of DMF $(15 \mathrm{ml})$ and water $(0.70 \mathrm{ml})$ and the resulted solution was stirred $15 \mathrm{~min}$ at $\mathrm{RT} . \mathrm{Pb}(\mathrm{OAc})_{4}(2.22 \mathrm{~g}, 0.005 \mathrm{~mol})$ was added and the mixture was further stirred for $30 \mathrm{~min}$. Water $(50 \mathrm{ml})$ was added and the mixture was extracted with EtOAc, combined organic layers were washed with water and brine, dried over anhydrous $\mathrm{Na}_{2} \mathrm{SO}_{4}$, concentrated under vacuum and submitted on flash chromatography $\left(\mathrm{SiO}_{2}\right.$, hex/EtOAc $\left.4: 1\right)$ afforded solid $(0.57 \mathrm{~g}, 55 \%)$.

In similar way below compound was obtained:

\begin{tabular}{|l|l|c|}
\hline Compound & \multicolumn{1}{|c|}{ Spectroscopic properties } & Yield: \\
\hline & $\begin{array}{l}\text { 2-(2-methoxyphenyl)ethene-1,1,2-tricarbonitrile } \\
\mathrm{mp}=93-95^{\circ} \mathrm{C}\end{array}$ & $55 \%$ \\
lit. $\mathrm{mp}=\mathrm{n} . \mathrm{d}$. & & \\
& $\begin{array}{l}1 \mathrm{H} \mathrm{NMR}(400 \mathrm{MHz}, \mathrm{DMSO}) \delta 7.76-7.71(\mathrm{~m}, 2 \mathrm{H}), 7.32(\mathrm{~d}, \mathrm{~J}=8.4 \mathrm{~Hz}, 1 \mathrm{H}), 7.21(\mathrm{td}, \mathrm{J}= \\
7.6,0.9 \mathrm{~Hz}, 1 \mathrm{H}), 3.96(\mathrm{~s}, 3 \mathrm{H})\end{array}$ & \\
\hline
\end{tabular}




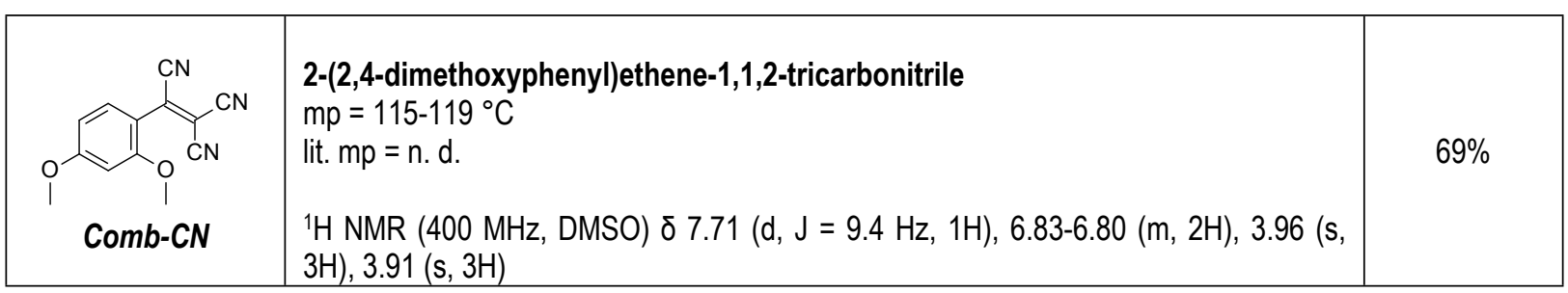

\section{Synthesis of new iodonium salts}

General procedure of synthesis of iodonium salts is describe in patent application P.426915 ("New diaryliodonium salts, methods of their preparation and use, new intermediate compounds for preparing new diaryliodonium salts and use of new intermediate compounds", date of application 2018-09-05). General synthetic procedure was described briefly below:<smiles>COC(=O)/C(N)=C/c1ccccc1OC</smiles>

2-[(2-methoxyphenyl)methylene]propanedinitrile $(1.00 \mathrm{~g}, \quad 0,005 \mathrm{~mol})$, diacetoxyiodobenzene $(1.925 \mathrm{~g}$, $0,006 \mathrm{~mol})$ and $\mathrm{KPF}_{6}(1.10 \mathrm{~g}, 0.006 \mathrm{~mol})$ were suspended in mixture of glacial acetic acid $(7.5 \mathrm{ml})$ and acetic anhydride $(3 \mathrm{ml})$, then concentrated $\mathrm{H}_{2} \mathrm{SO}_{4}(0.305 \mathrm{ml}, 0.006 \mathrm{~mol})$ was added drop wise and the mixture was stirred for $1 \mathrm{~h}$ at $50^{\circ} \mathrm{C}$. After that the mixture was cooled to RT and water $(50 \mathrm{ml})$ was added. Formed precipitate was filtered off, washed with water and $\mathrm{Et}_{2} \mathrm{O}$, then dried in the air. Slightly yellow solid was obtained $(2.25 \mathrm{~g}$, $78 \%)$.

Obtained new iodonium salts:

\begin{tabular}{|c|c|c|}
\hline Compound & Spectroscopic properties & Yield: \\
\hline$\alpha-C N-P I$ & $\begin{array}{l}\text { [3-(2,2-dicyanovinyl)-4-methoxy-phenyl]-phenyl-iodonium } \\
\text { hexafluorophosphate } \\
\mathrm{mp}=186-190{ }^{\circ} \mathrm{C} \\
{ }^{1} \mathrm{H} \mathrm{NMR}(400 \mathrm{MHz}, \mathrm{DMSO}) \delta 8.67(\mathrm{~d}, \mathrm{~J}=2.2 \mathrm{~Hz}, 1 \mathrm{H}), 8.51(\mathrm{~s}, 1 \mathrm{H}), 8.49(\mathrm{dd}, \mathrm{J} \\
=9.0,2.2 \mathrm{~Hz}, 1 \mathrm{H}), 8.24-8.21(\mathrm{~m}, 2 \mathrm{H}), 7.66(\mathrm{tt}, \mathrm{J}=7.5,1.0 \mathrm{~Hz}, 1 \mathrm{H}), 7.54-7.49 \\
(\mathrm{~m}, 2 \mathrm{H}), 7.35(\mathrm{~d}, \mathrm{~J}=9.2 \mathrm{~Hz}, 1 \mathrm{H}), 3.94(\mathrm{~s}, 3 \mathrm{H}) \\
{ }^{3} \mathrm{C} \mathrm{NMR}(101 \mathrm{MHz}, \mathrm{DMSO}) \delta 160.91,156.09,142.44,136.63,135.40, \\
132.51,132.22,123.33,117.64,116.14,114.09,112.93,106.20,85.89,57.28 \\
\text { MS (ESI) } \mathrm{m} / \mathrm{z}(\%): 387\left([\mathrm{M}+\mathrm{H}]^{+}, 100 \%\right) \\
\text { Purity (LC): } 97,5 \%\end{array}$ & $78 \%$ \\
\hline$\alpha-\mathrm{SO}_{2} \mathrm{Me}-\mathrm{PI}$ & $\begin{array}{l}\text { [3-[(E)-2-cyano-2-methylsulfonyl-vinyl]-4-methoxy-phenyl]-phenyl- } \\
\text { iodonium hexafluorophosphate } \\
\text { mp = } 170-172^{\circ} \mathrm{C} \\
{ }^{1} \mathrm{H} \mathrm{NMR}(400 \mathrm{MHz}, \mathrm{DMSO}) \delta 8.76(\mathrm{~d}, \mathrm{~J}=1.4 \mathrm{~Hz}, 1 \mathrm{H}), 8.50(\mathrm{dd}, \mathrm{J}=8.8,1.9 \\
\mathrm{Hz}, 1 \mathrm{H}), 8.33(\mathrm{~s}, 1 \mathrm{H}), 8.25(\mathrm{~d}, \mathrm{~J}=8.0 \mathrm{~Hz}, 2 \mathrm{H}), 7.67(\mathrm{t}, \mathrm{J}=7.5 \mathrm{~Hz}, 1 \mathrm{H}), 7.53(\mathrm{t}, \\
\mathrm{J}=7.9 \mathrm{~Hz}, 2 \mathrm{H}), 7.38(\mathrm{~d}, \mathrm{~J}=8.8 \mathrm{~Hz}, 1 \mathrm{H}), 3.97(\mathrm{~s}, 3 \mathrm{H}), 3.42(\mathrm{~s}, 3 \mathrm{H}) \\
{ }^{13} \mathrm{C} \mathrm{NMR}(101 \mathrm{MHz}, \mathrm{DMSO}) \delta 161.31,146.95,142.38,136.56,135.46, \\
132.53,132.22,122.10,117.65,116.23,113.23,106.33,57.36,42.24 \\
\text { MS (ESI) } \mathrm{m} / \mathrm{z}(\%): 440\left([\mathrm{M}+\mathrm{H}]^{+}, 100 \%\right) \\
\text { Purity (LC): } 97,8 \%\end{array}$ & $77 \%$ \\
\hline
\end{tabular}




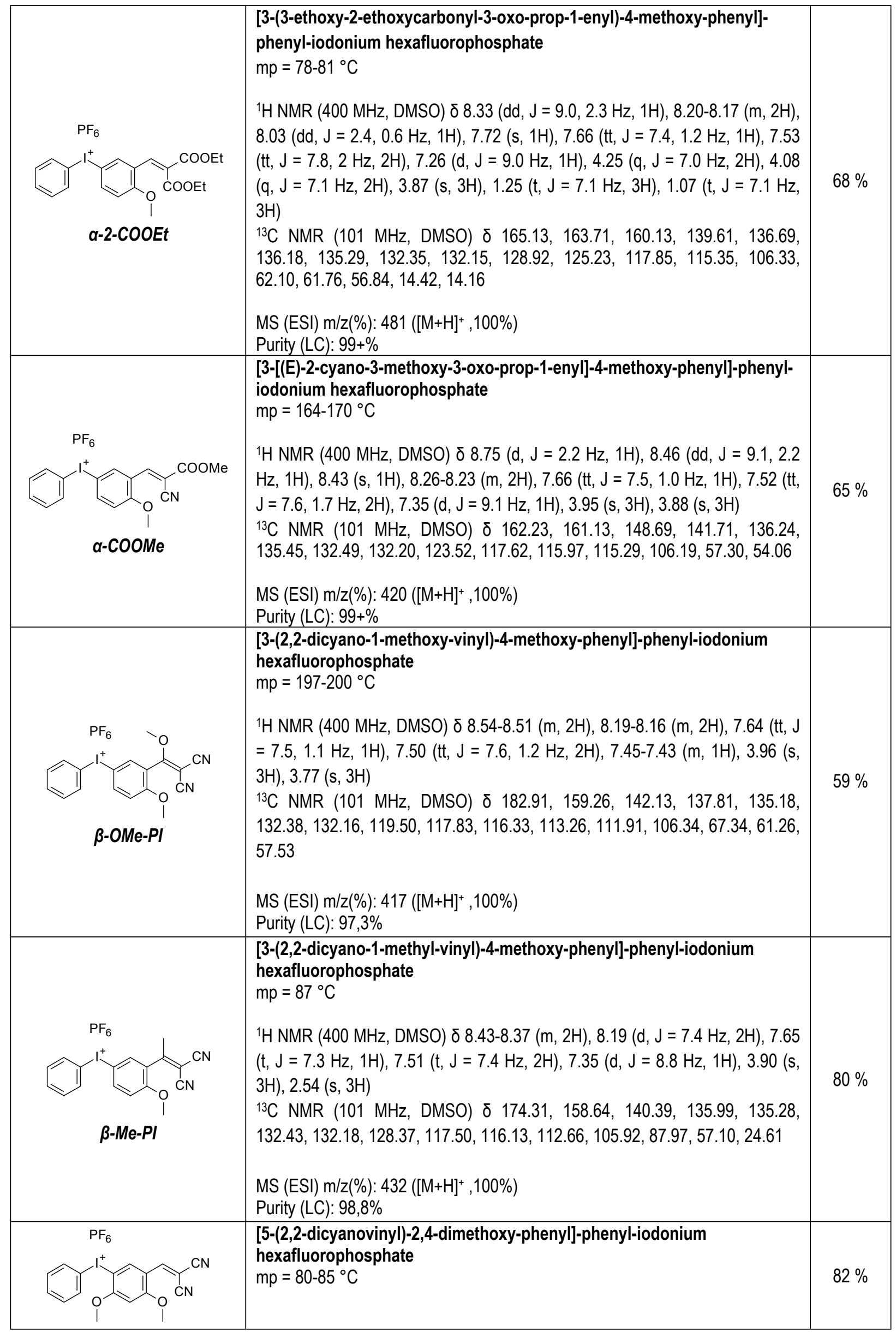




\begin{tabular}{|c|c|c|}
\hline$A r-2,4-O M e-P \mid$ & $\begin{array}{l}{ }^{1} \mathrm{H} \text { NMR }(400 \mathrm{MHz}, \mathrm{DMSO}) \delta 8.79(\mathrm{~s}, 1 \mathrm{H}), 8.35(\mathrm{~s}, 1 \mathrm{H}), 8.14-8.11(\mathrm{~m}, 2 \mathrm{H}), 7.65 \\
(\mathrm{tt}, \mathrm{J}=7.4,1.1 \mathrm{~Hz}, 1 \mathrm{H}), 7.52-7.47(\mathrm{~m}, 2 \mathrm{H}), 6.96(\mathrm{~s}, 1 \mathrm{H}), 4.09(\mathrm{~s}, 3 \mathrm{H}), 4.01(\mathrm{~s}, \\
3 \mathrm{H}) \\
{ }^{13 \mathrm{C}} \mathrm{NMR}(101 \mathrm{MHz}, \mathrm{DMSO}) \delta 164.37,162.99,154.66,138.65,135.42, \\
\text { 132.44, } 132.13,116.99,115.82,114.82,113.74,97.99,97.70,81.25,65.37, \\
58.61,57.55,15.63 \\
\text { MS (ESI) } \mathrm{m} / \mathrm{z}(\%): 417\left([\mathrm{M}+\mathrm{H}]^{+}, 100 \%\right) \\
\text { Purity (LC): } 99+\%\end{array}$ & \\
\hline Comb-Me & $\begin{array}{l}\text { [5-(2,2-dicyano-1-methyl-vinyl)-2,4-dimethoxy-phenyl]-phenyl-iodonium } \\
\text { hexafluorophosphate } \\
\text { mp }=92{ }^{\circ} \mathrm{C} \\
{ }^{1} \mathrm{H} \mathrm{NMR}(400 \mathrm{MHz}, \mathrm{DMSO}) \delta 8.48(\mathrm{~s}, 1 \mathrm{H}), 8.11-8.07(\mathrm{~m}, 2 \mathrm{H}), 7.66(\mathrm{tt}, \mathrm{J}=7.5 \text {, } \\
1.4 \mathrm{~Hz}, 1 \mathrm{H}), 7.51(\mathrm{tt}, \mathrm{J}=7.5,1.4 \mathrm{~Hz}, 2 \mathrm{H}), 6.98(\mathrm{~s}, 1 \mathrm{H}), 4.07(\mathrm{~s}, 3 \mathrm{H}), 3.98(\mathrm{~s}, \\
3 \mathrm{H}), 2.55(\mathrm{~s}, 3 \mathrm{H}) \\
{ }^{13} \mathrm{C} \mathrm{NMR}(101 \mathrm{MHz}, \mathrm{DMSO}) \delta 173.82,161.66,161.02,137.88,135.25, \\
132.30,132.08,120.30,116.99,113.16,98.25,96.81,86.66,58.26,57.22, \\
24.72 \\
\text { MS (ESI) } \mathrm{m} / \mathrm{z}(\%): 431\left([\mathrm{M}+\mathrm{H}]^{+}, 100 \%\right) \\
\text { Purity (LC): } 99+\%\end{array}$ & $81 \%$ \\
\hline Comb-OMe-PI & 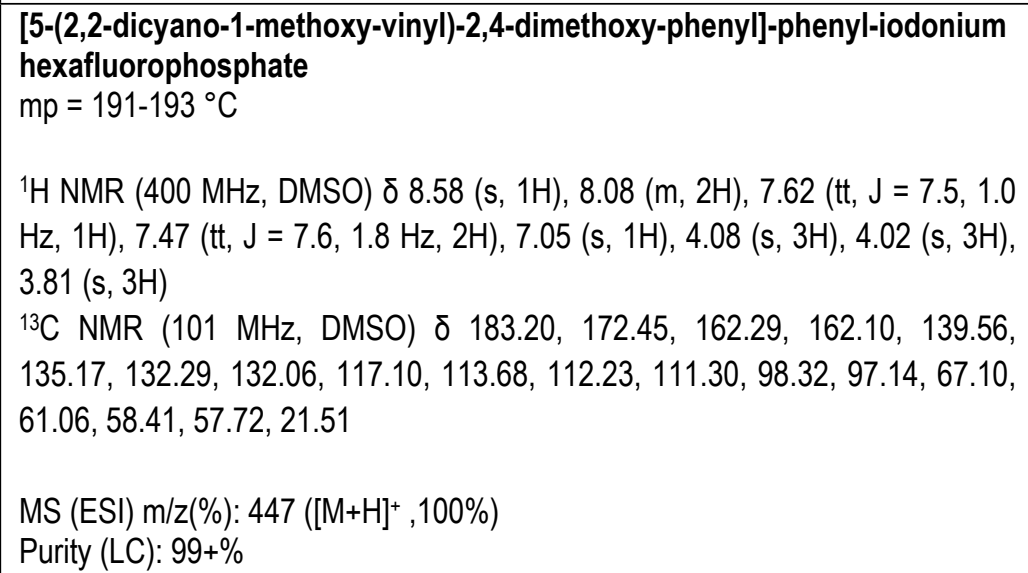 & $48 \%$ \\
\hline & 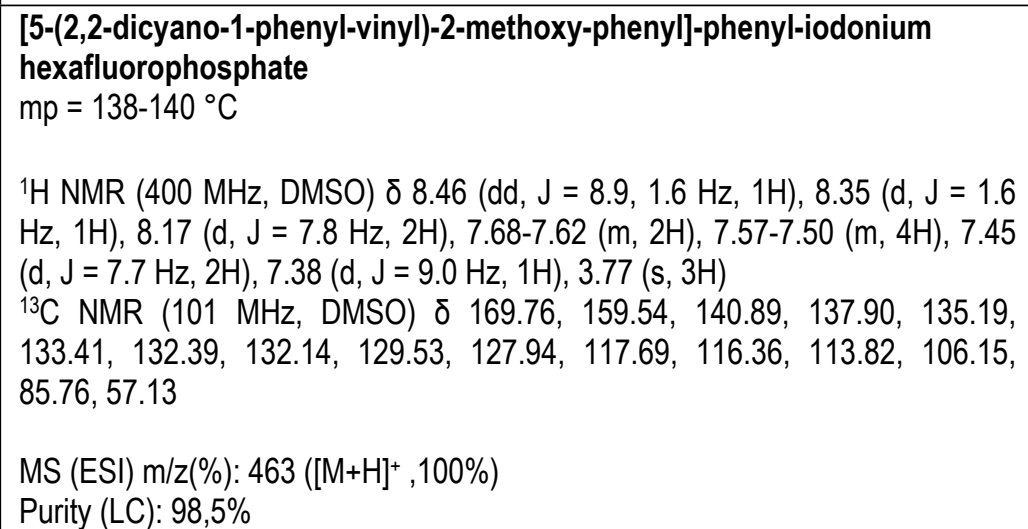 & $66 \%$ \\
\hline Comb-Ph-PI & $\begin{array}{l}\text { [5-(2,2-dicyano-1-phenyl-vinyl)-2,4-dimethoxy-phenyl]-phenyl-iodonium } \\
\text { hexafluorophosphate } \\
\mathrm{mp}=115-117^{\circ} \mathrm{C} \\
{ }^{1} \mathrm{H} \text { NMR }(400 \mathrm{MHz}, \mathrm{DMSO}) \delta 8.38(\mathrm{~s}, 1 \mathrm{H}), 8.08-8.05(\mathrm{~m}, 2 \mathrm{H}), 7.68-7.62(\mathrm{~m} \text {, } \\
2 \mathrm{H}), 7.56(\mathrm{tt}, \mathrm{J}=7.5,1.6 \mathrm{~Hz}, 2 \mathrm{H}), 7.52-7.45(\mathrm{~m}, 4 \mathrm{H}), 7.01(\mathrm{~s}, 1 \mathrm{H}), 4.09(\mathrm{~s}, 3 \mathrm{H}) \text {, } \\
3.82(\mathrm{~s}, 3 \mathrm{H})\end{array}$ & $73 \%$ \\
\hline
\end{tabular}




\begin{tabular}{|c|c|c|}
\hline & $\begin{array}{l}{ }^{13} \mathrm{C} \mathrm{NMR}(101 \mathrm{MHz}, \mathrm{DMSO}) \delta 169.85,162.77,161.53,140.15,135.90, \\
135.21,133.27,132.32,129.83,129.44,119.89,117.01,114.35,98.39,96.90, \\
84.28,58.31,57.24 \\
\text { MS (ESI) } \mathrm{m} / \mathrm{z}(\%): 493\left([\mathrm{M}+\mathrm{H}]^{+}, 100 \%\right) \\
\text { Purity (LC): } 99 \%\end{array}$ & \\
\hline Comb-CN-PI & 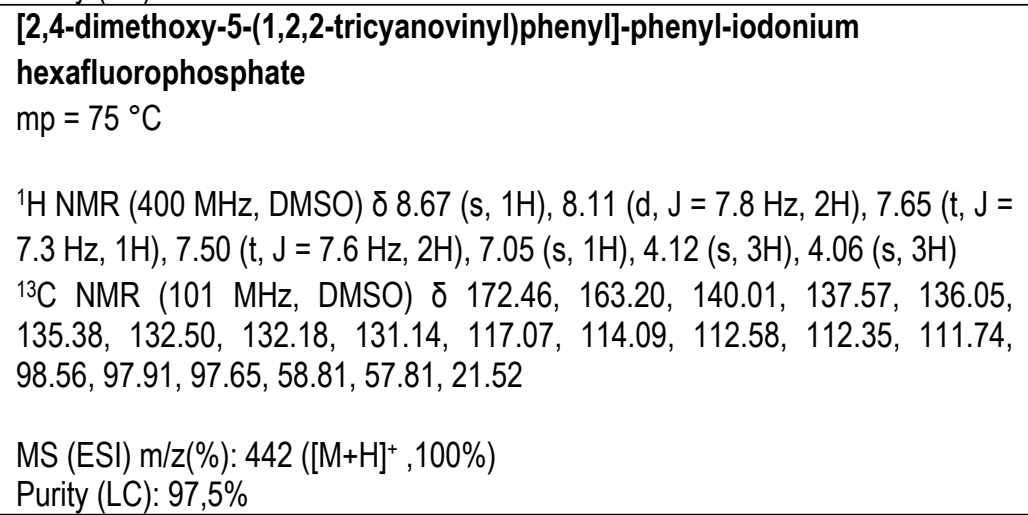 & $63 \%$ \\
\hline$A r-2,6-O M e-P I$ & 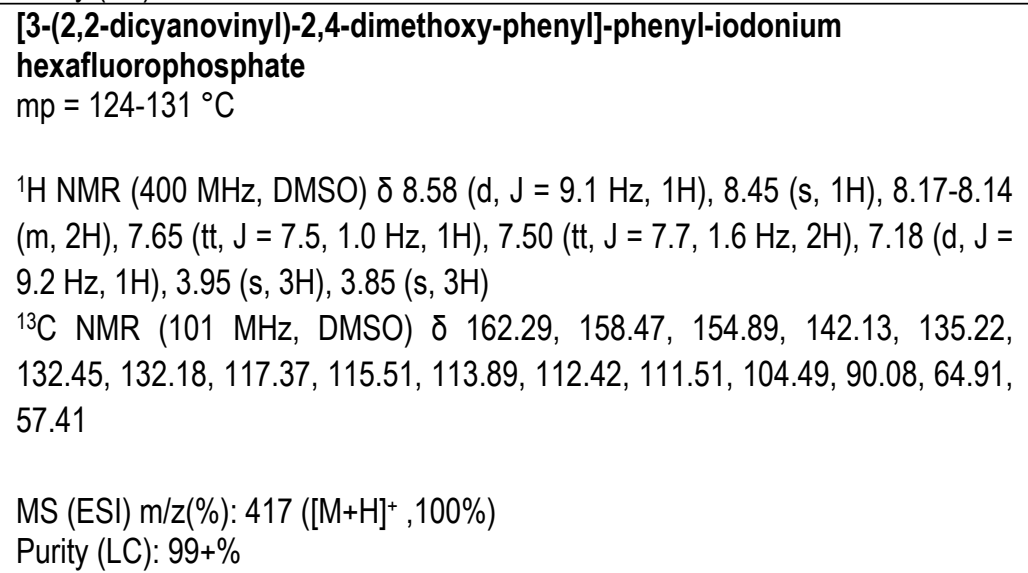 & $49 \%$ \\
\hline$A r-2,4,6-O M e-P I$ & 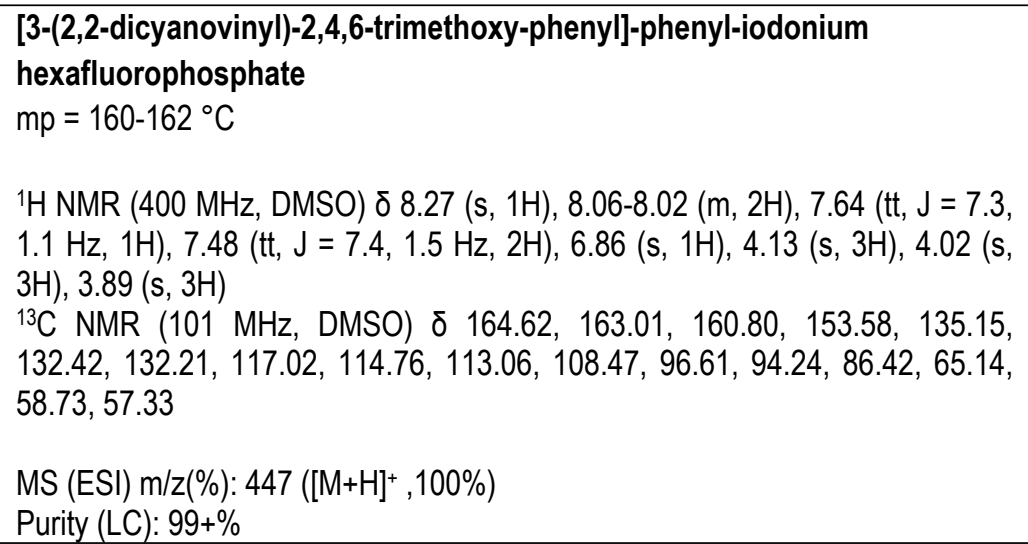 & $66 \%$ \\
\hline
\end{tabular}


10. ${ }^{1} \mathrm{H}$ NMR and ${ }^{13} \mathrm{C}$ NMR spectra of synthesized compounds

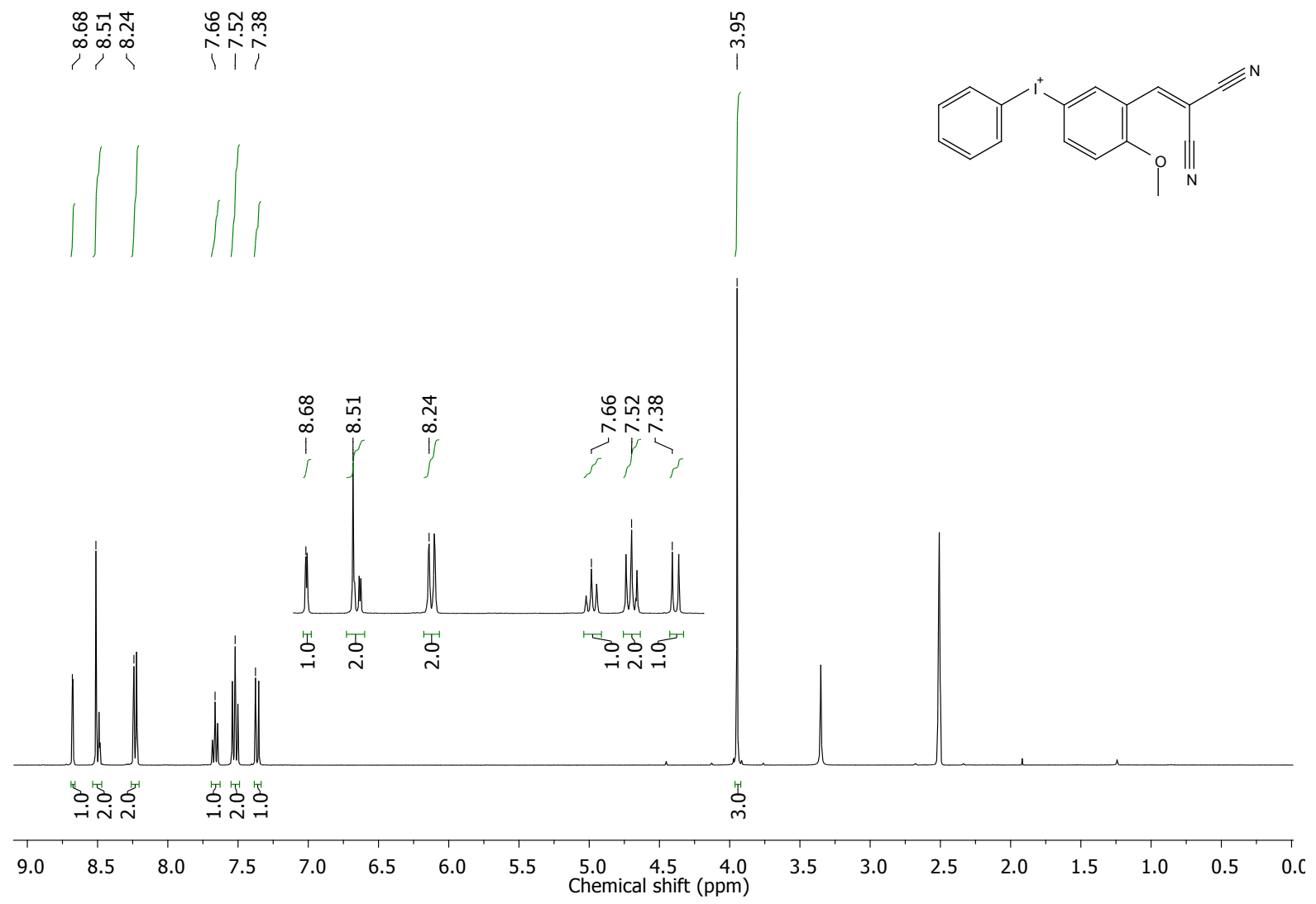

Figure S2. ${ }^{1} \mathrm{H}$ NMR of $\alpha-C N-P I$.

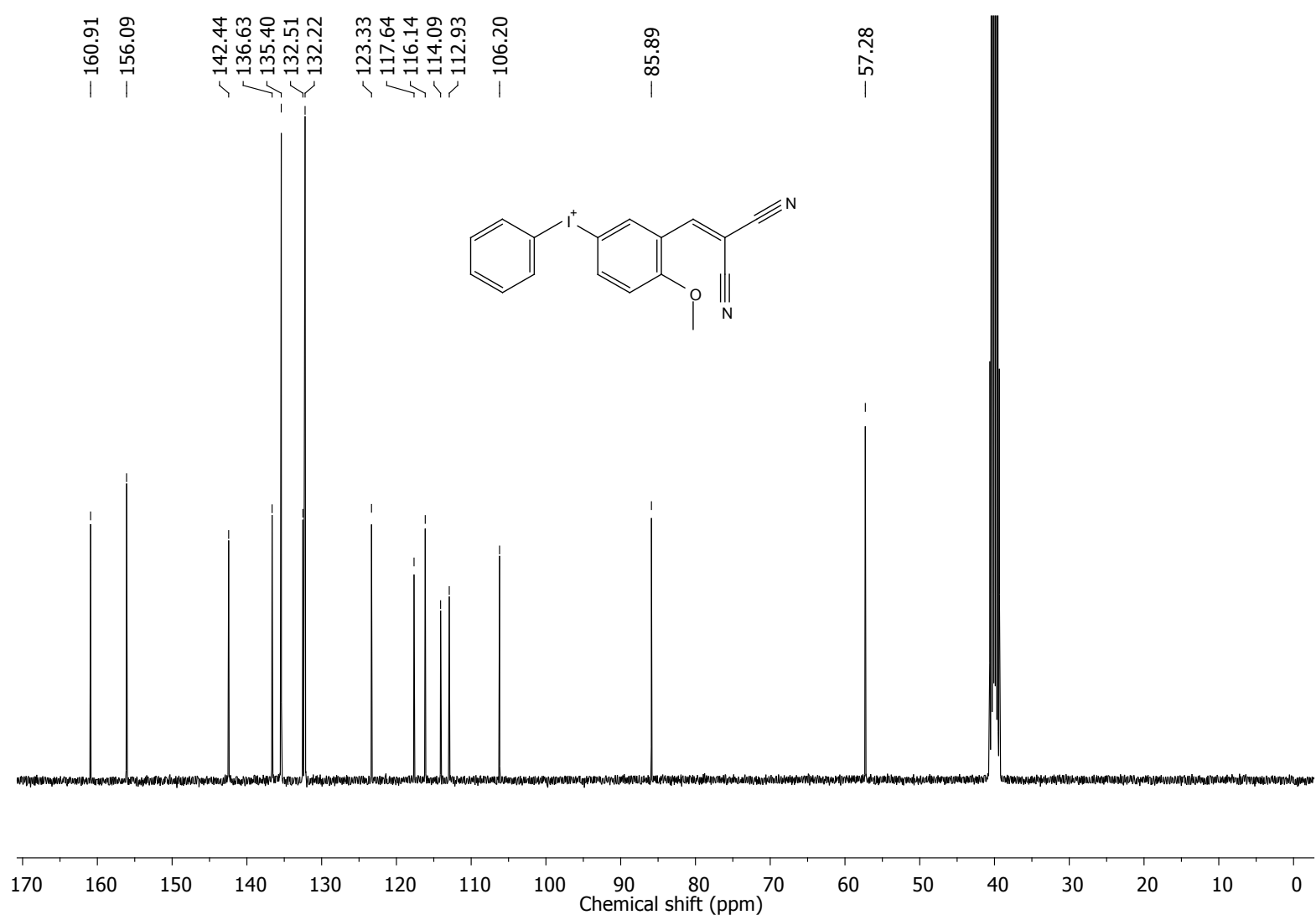

Figure S3. ${ }^{13} \mathrm{C}$ NMR of $\alpha-C N-P I$. 


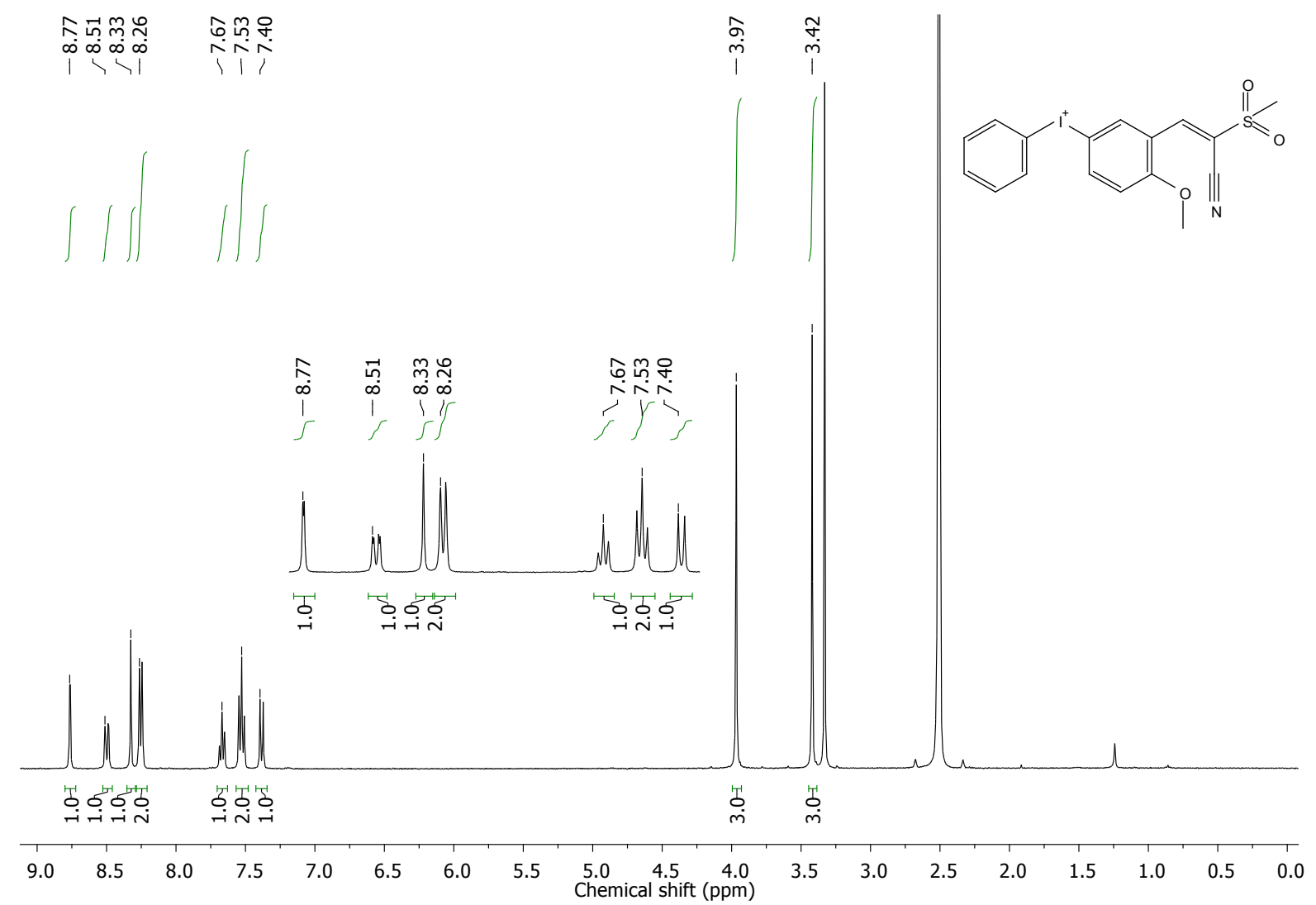

Figure S4. ${ }^{1} \mathrm{H} \mathrm{NMR}$ of $\alpha-\mathrm{SO}_{2} \mathrm{Me}-\mathrm{PI}$.

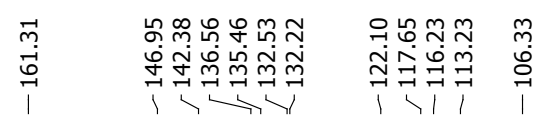
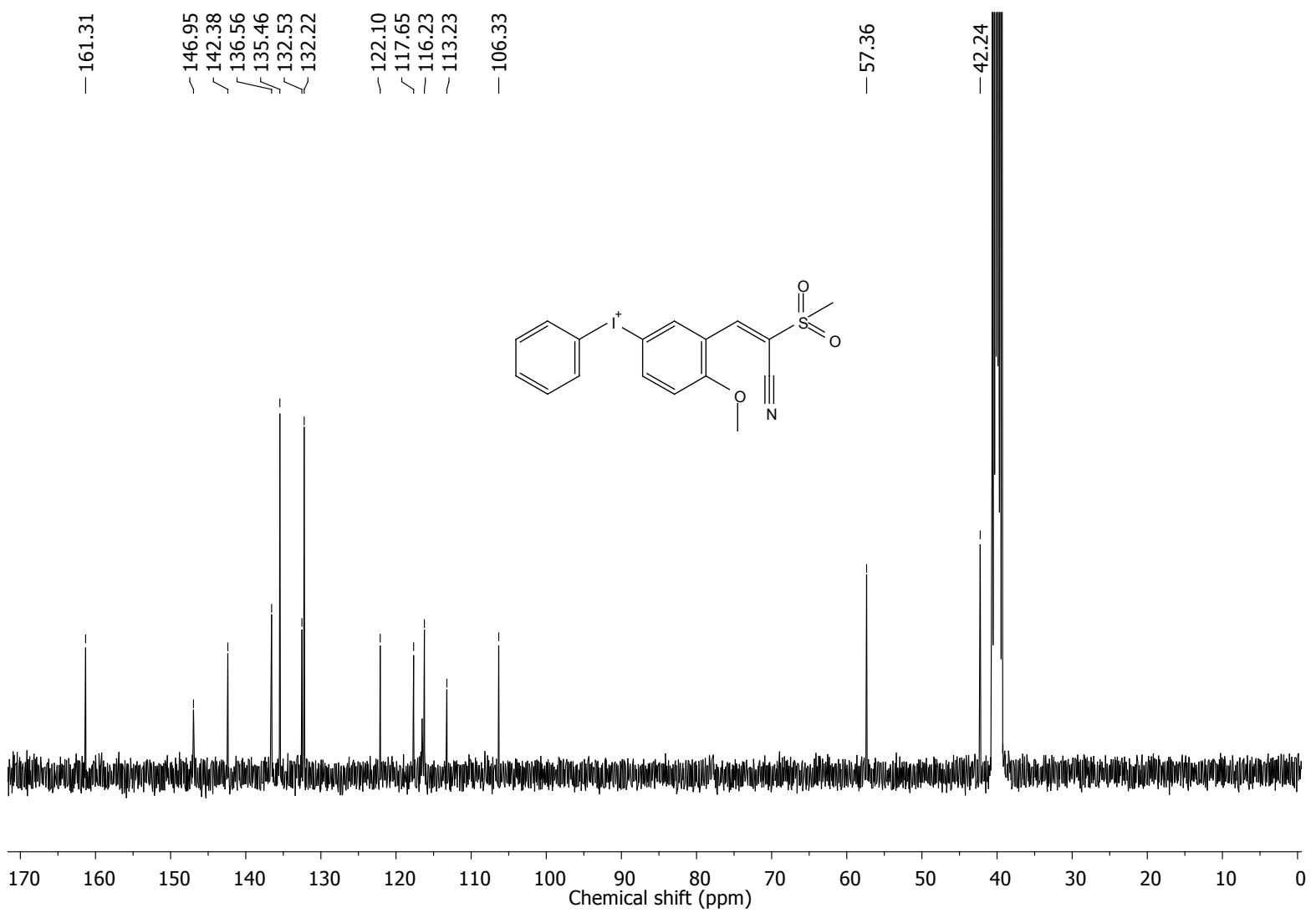

Figure S5. ${ }^{13} \mathrm{C} \mathrm{NMR} \mathrm{a}_{-} \mathrm{SO}_{2} \mathrm{Me}-\mathrm{PI}$. 


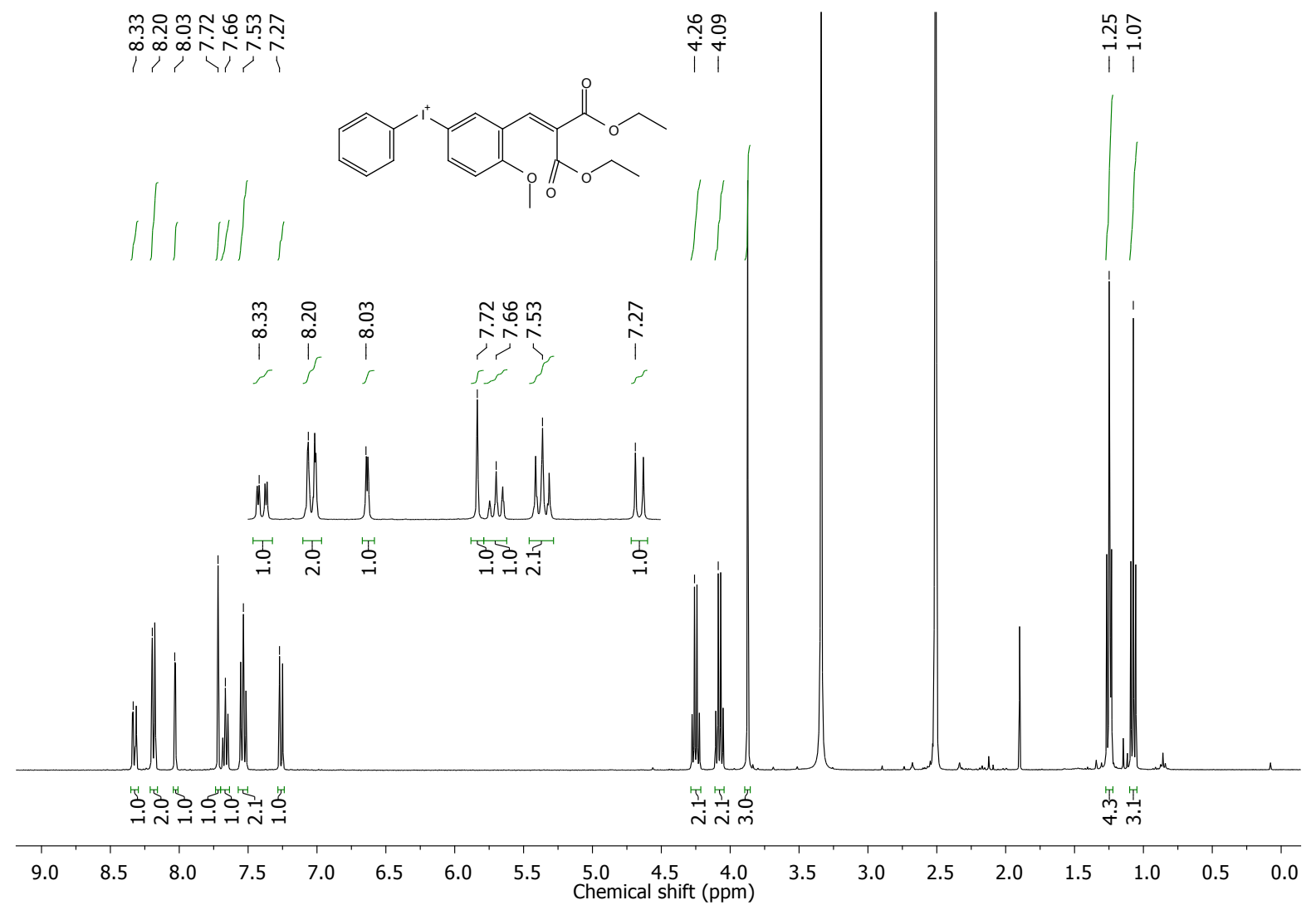

Figure S6. ${ }^{1} \mathrm{H}$ NMR of $\alpha-2$-COOEt.

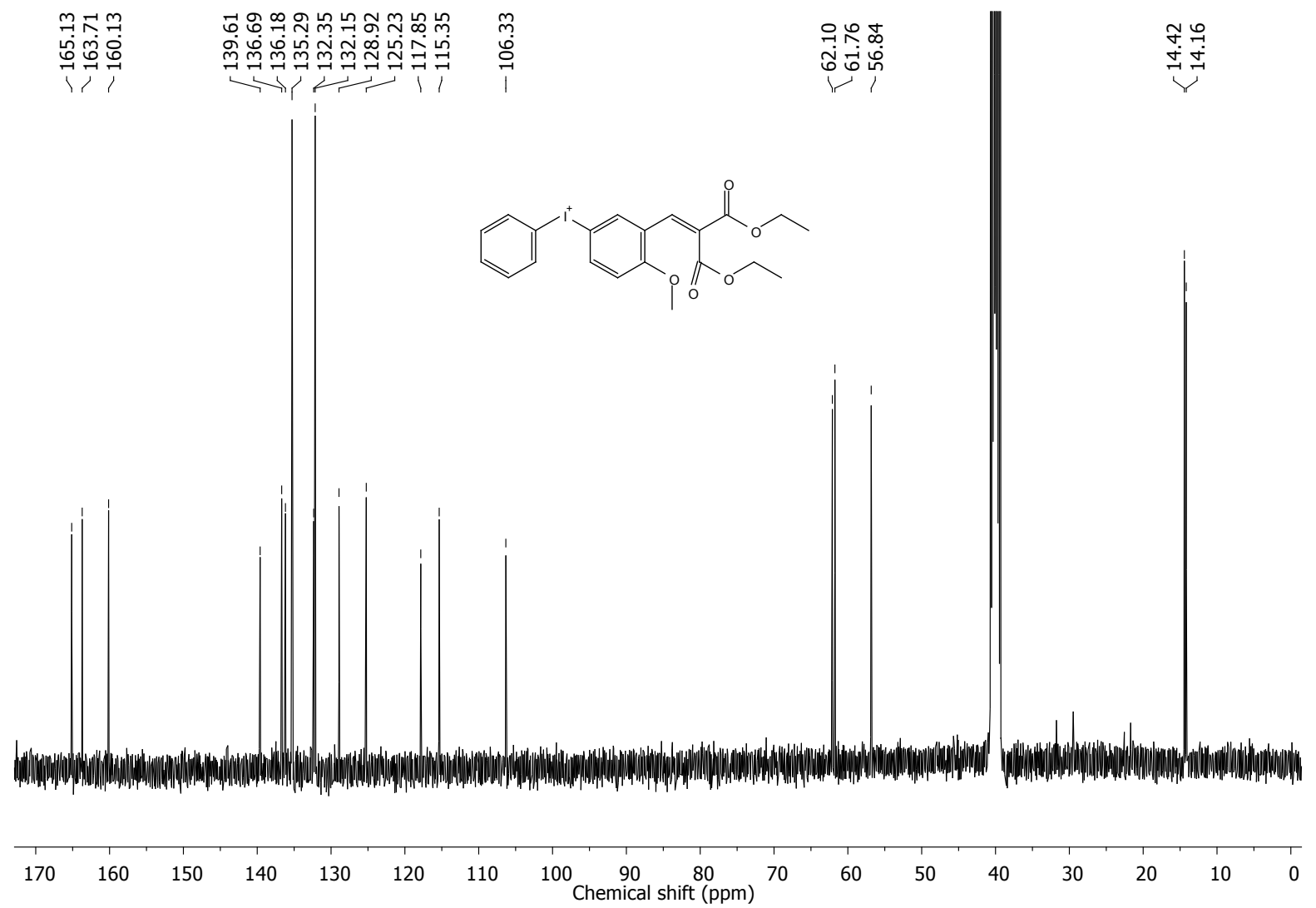

Figure S7. ${ }^{13} \mathrm{C}$ NMR of $\alpha-2$-COOEt. 


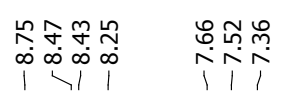
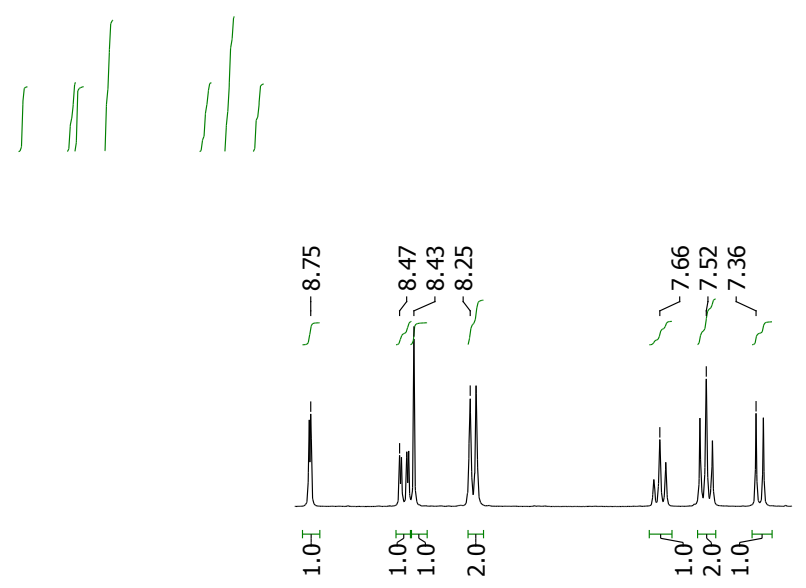

인

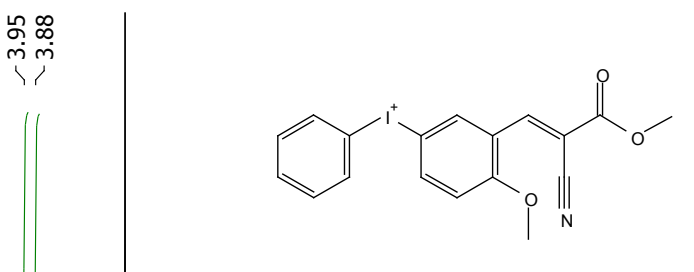

Figure S8. ${ }^{1} \mathrm{H}$ NMR of $\alpha$-COOMe.

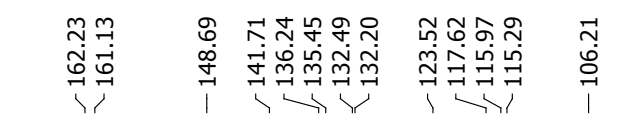
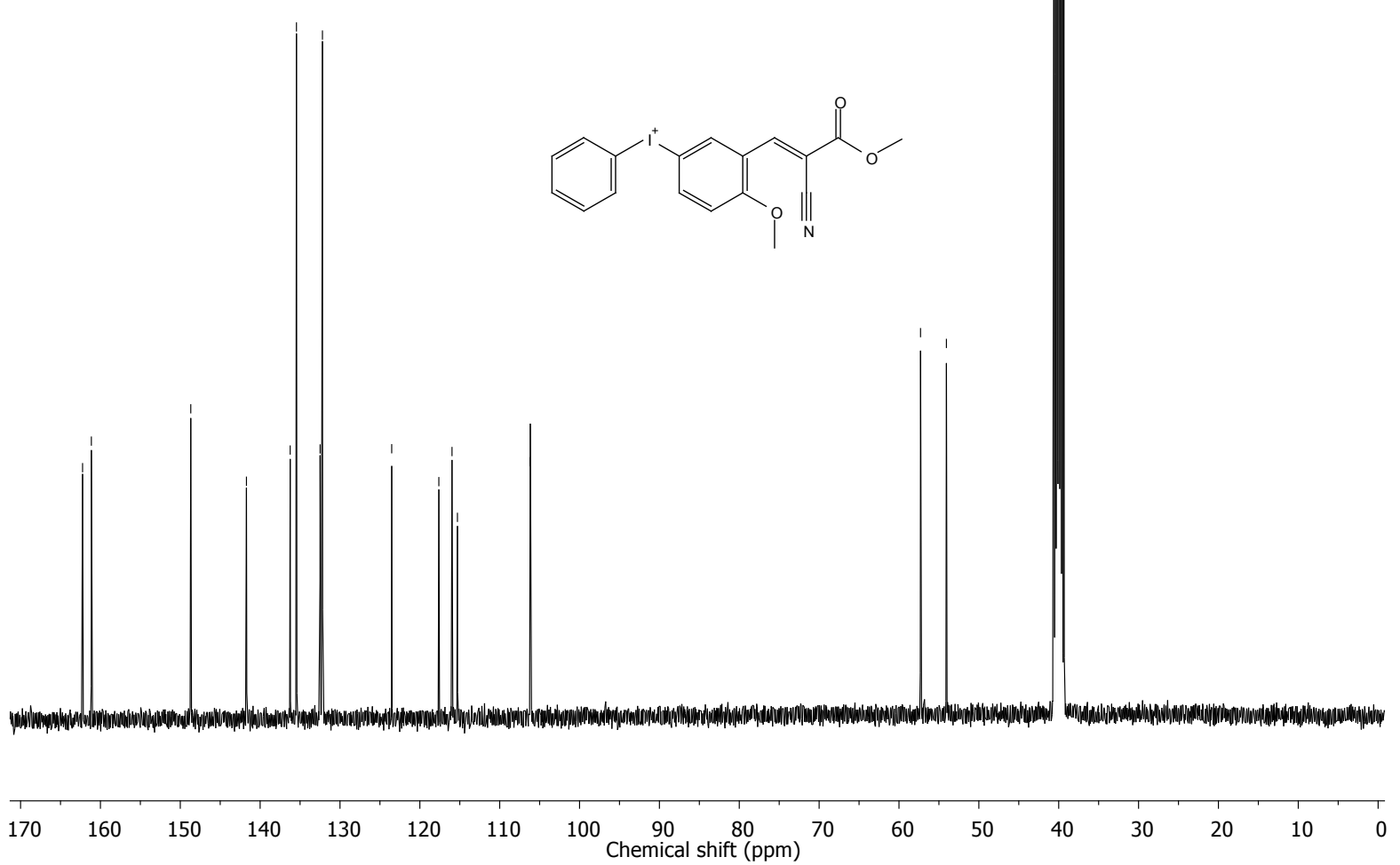

Figure S9. ${ }^{13} \mathrm{C}$ NMR of $\alpha-C O O M e$. 

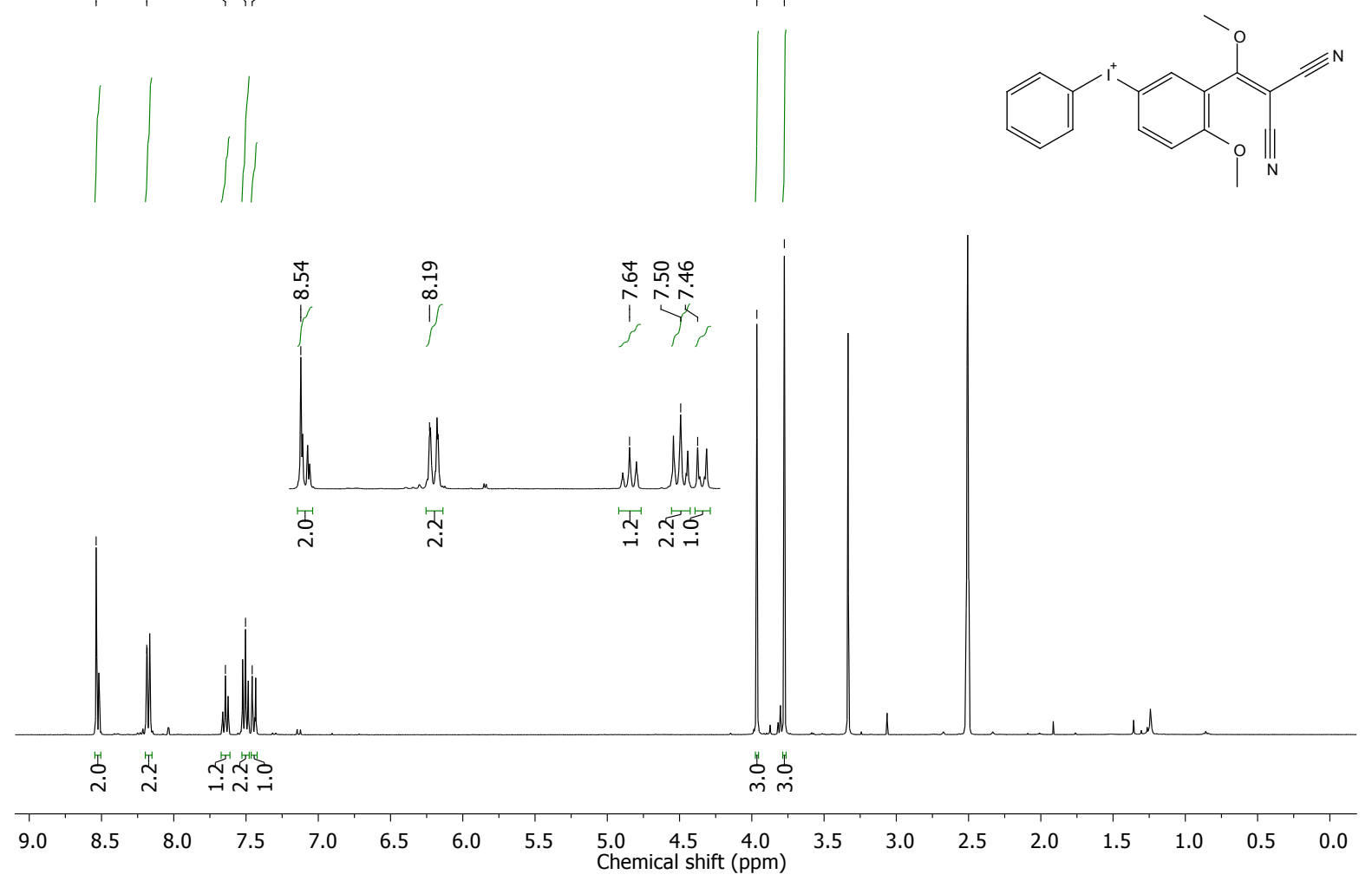

Figure S10. ${ }^{1} \mathrm{H}$ NMR of $\beta$-OMe-PI.

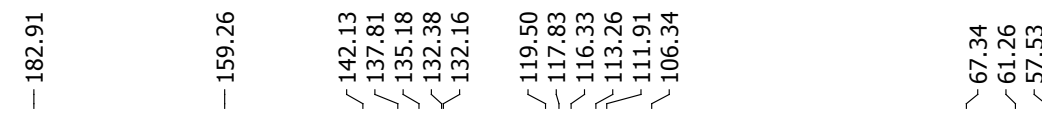
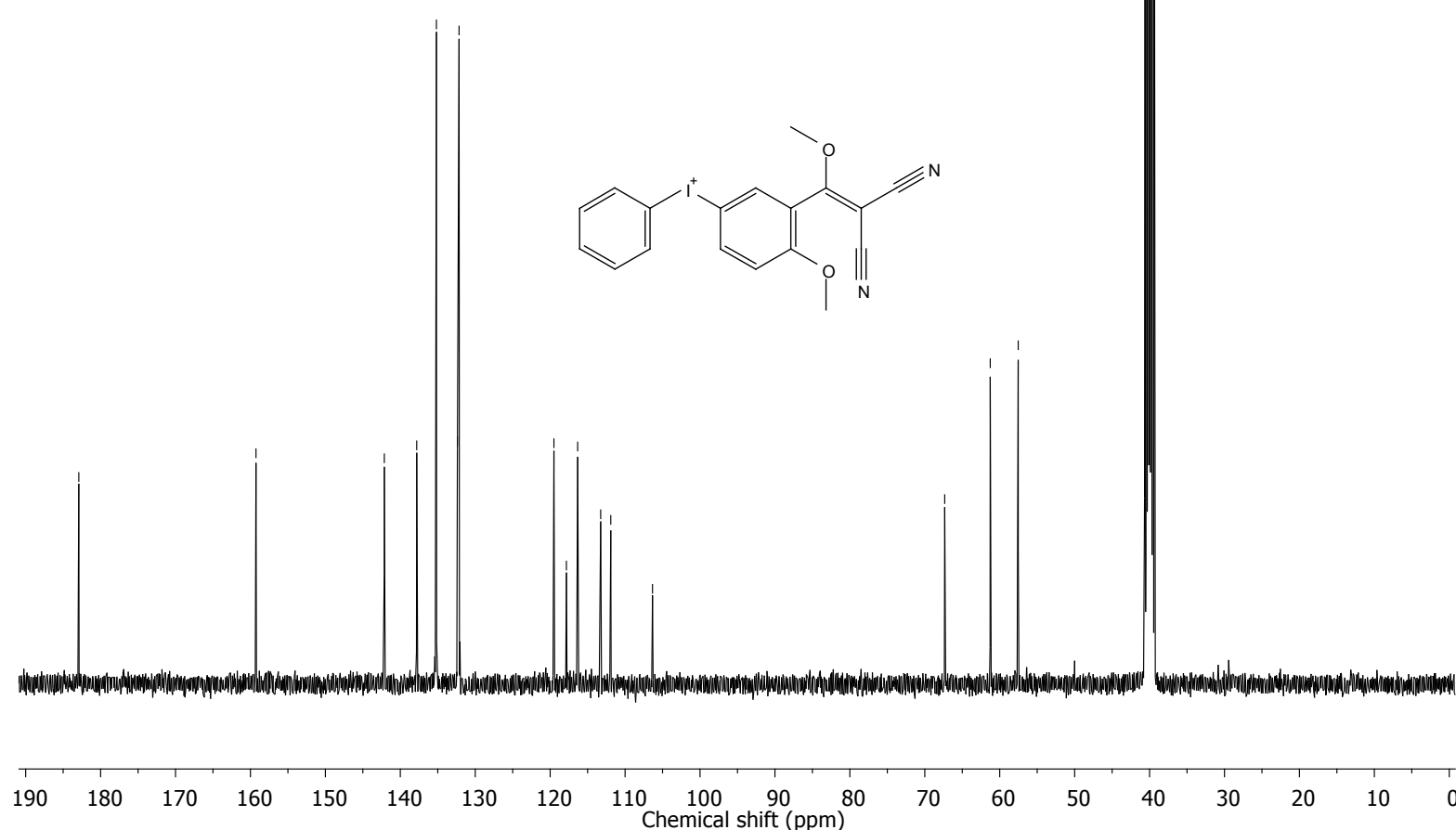

Figure S11. ${ }^{13} \mathrm{C}$ NMR of $\beta$-OMe-PI. 


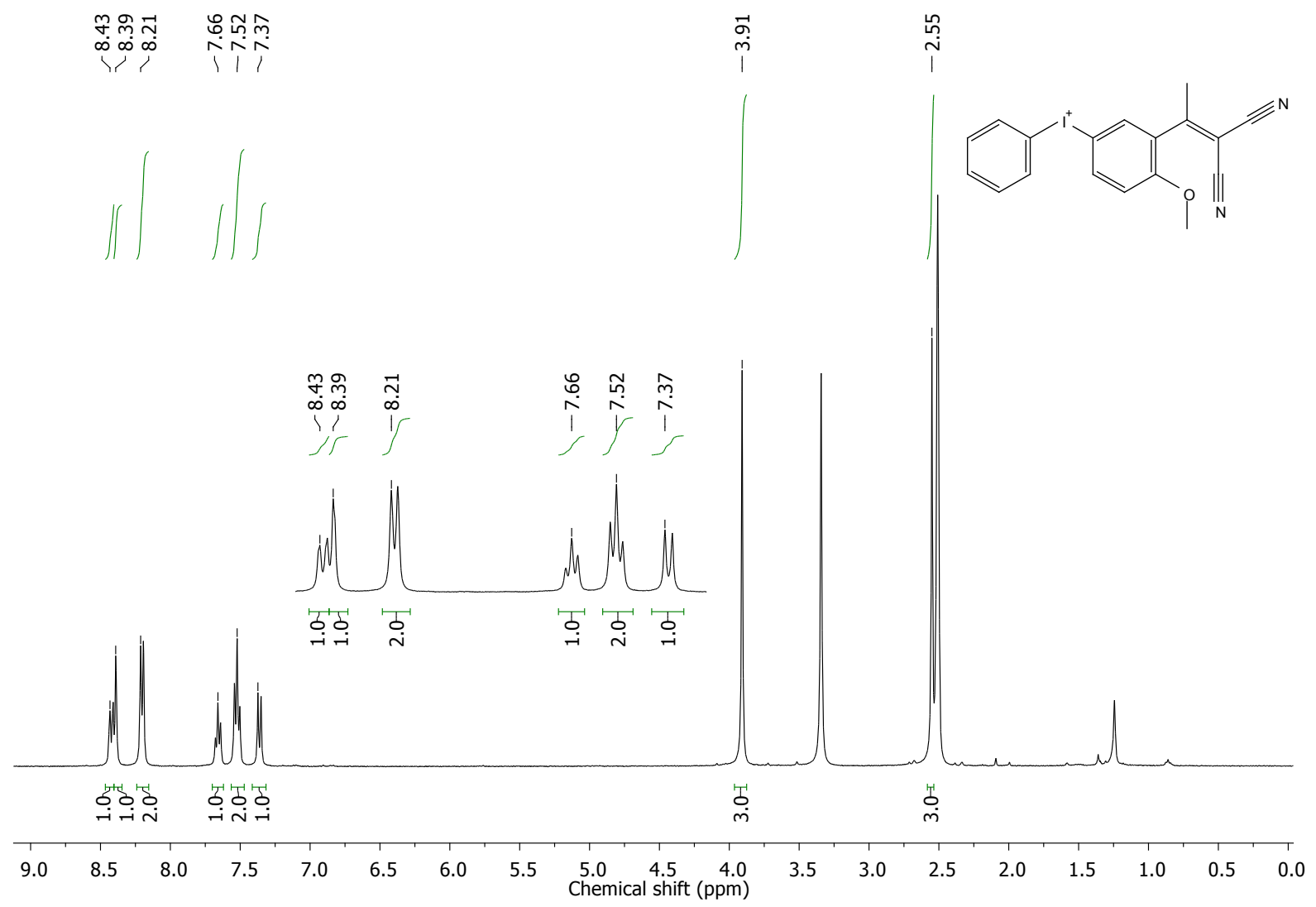

Figure S12. ${ }^{1} \mathrm{H}$ NMR of $\beta-M e-P I$.
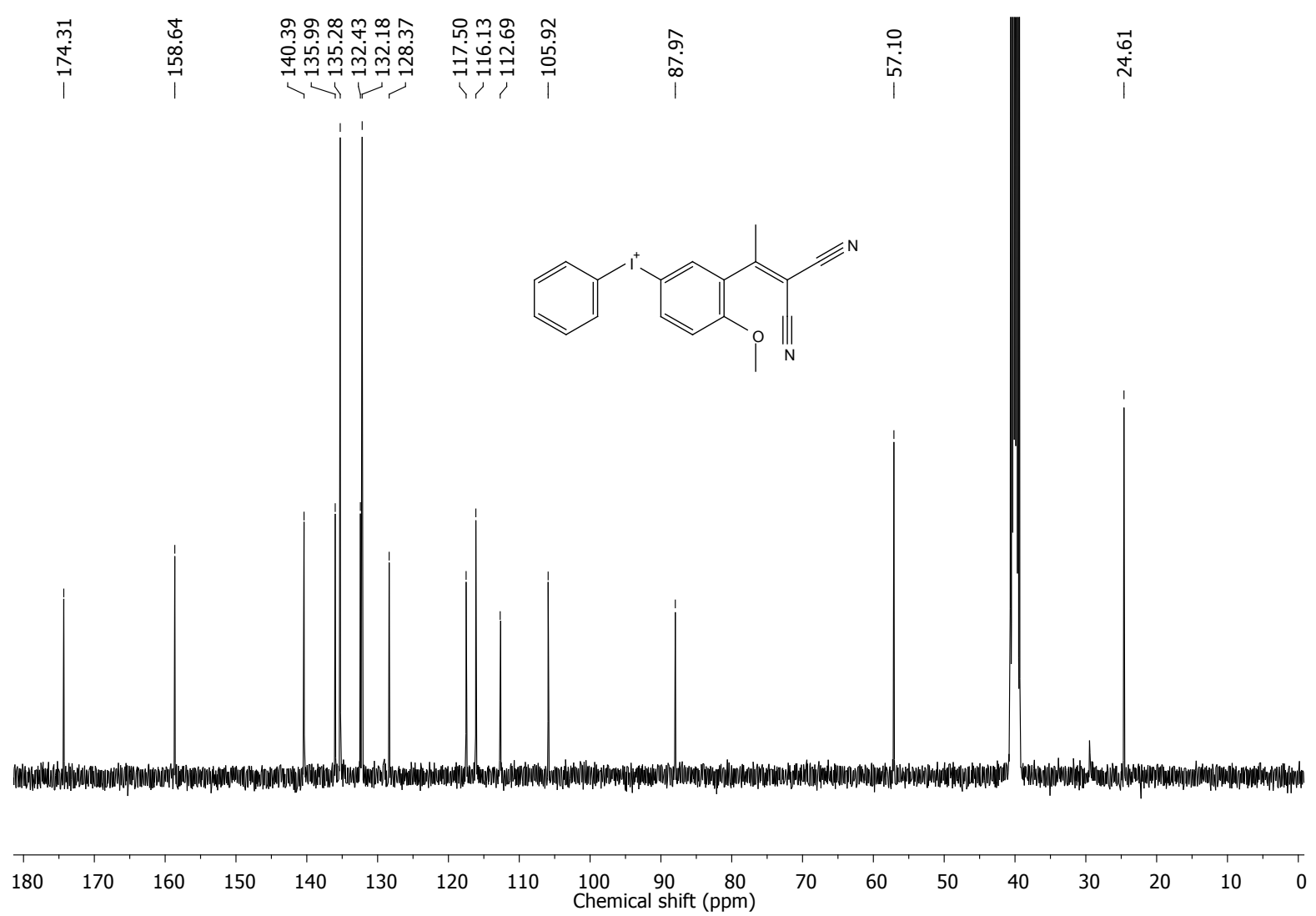

Figure S13. ${ }^{13} \mathrm{C}$ NMR of $\beta$-Me-PI. 

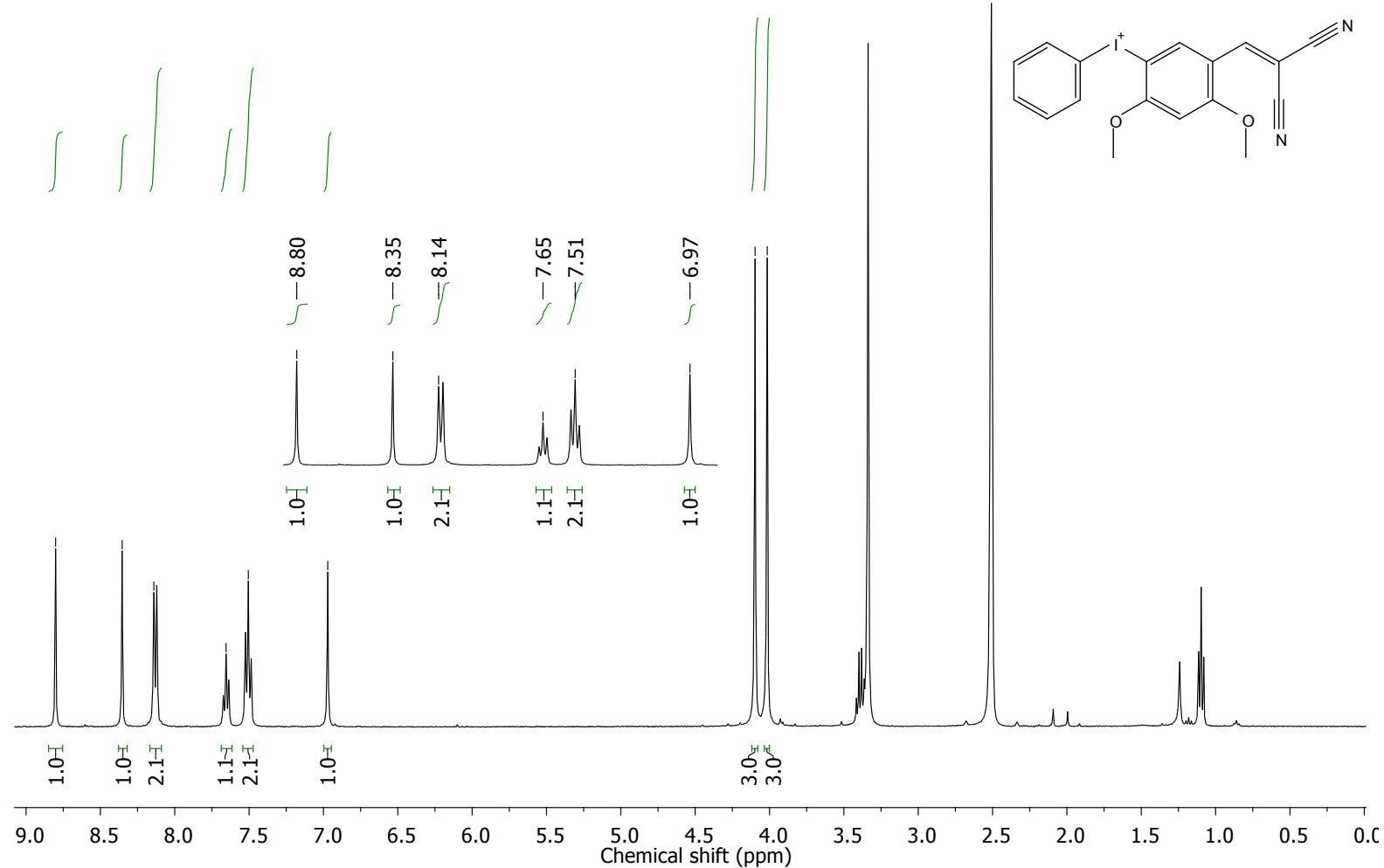

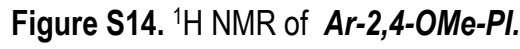

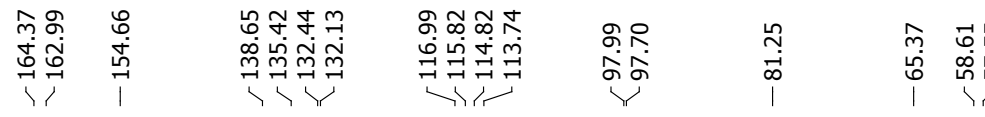<smiles>COc1cc(OC)c([I-]c2ccccc2)cc1C=C(C#N)C#N</smiles>
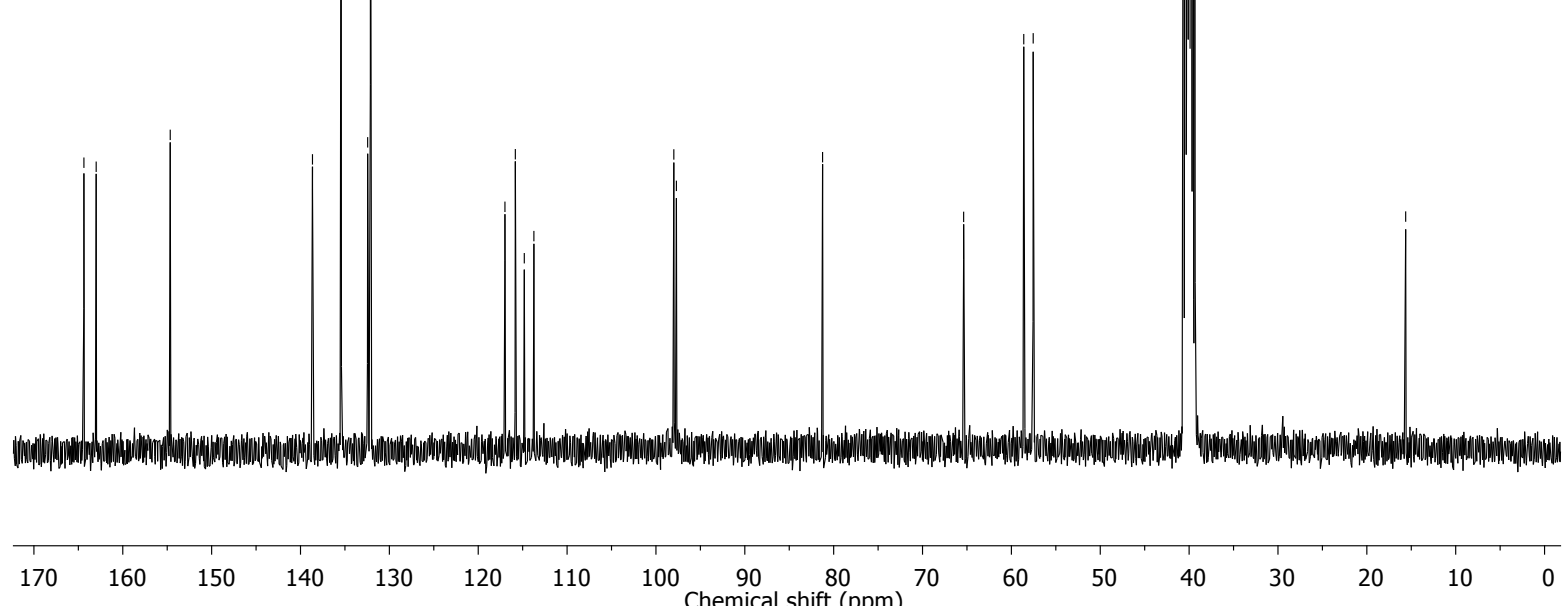

Figure S15. ${ }^{13} \mathrm{C}$ NMR of $A r-2,4-O M e-P I$. 


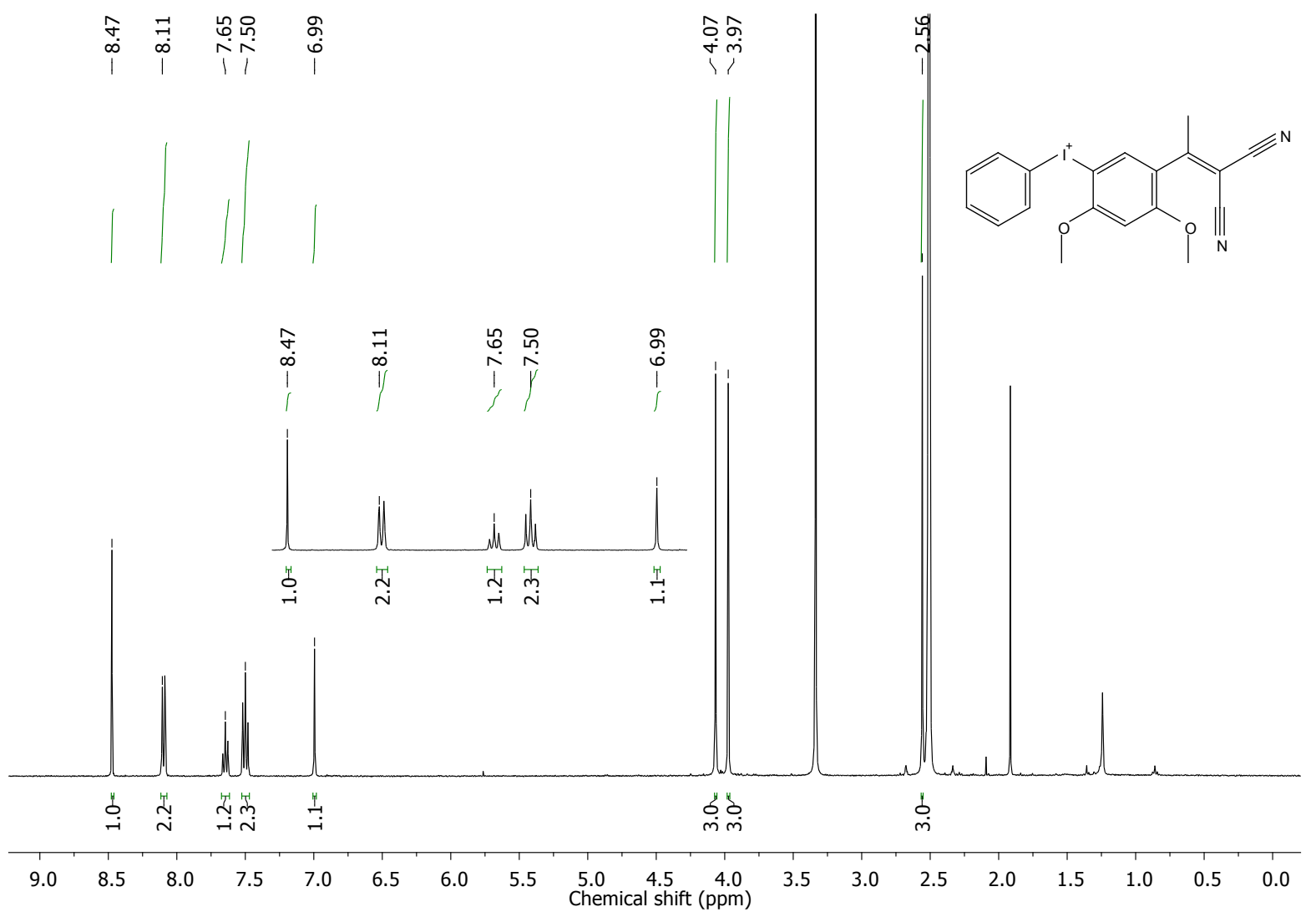

Figure S16. ${ }^{1} \mathrm{H}$ NMR of Comb-Me-PI.
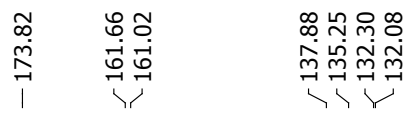

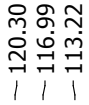

No
$\infty$
$\infty$
$\infty$
1
1

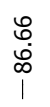

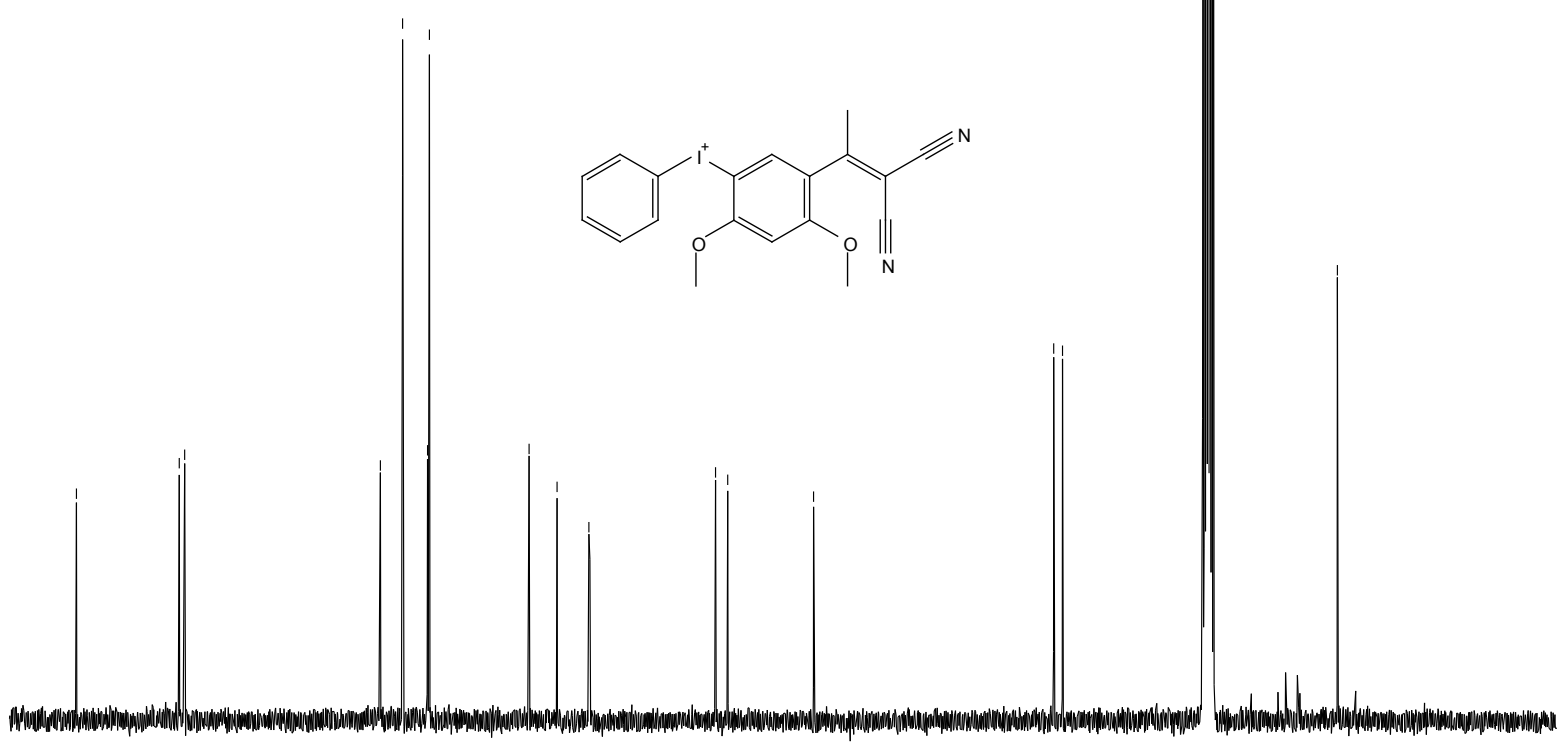

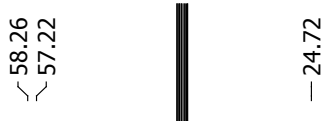

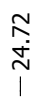

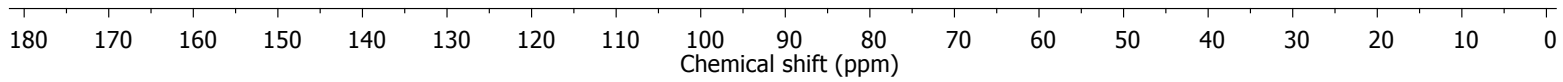

Figure S17. ${ }^{13} \mathrm{C}$ NMR of Comb-Me-PI. 


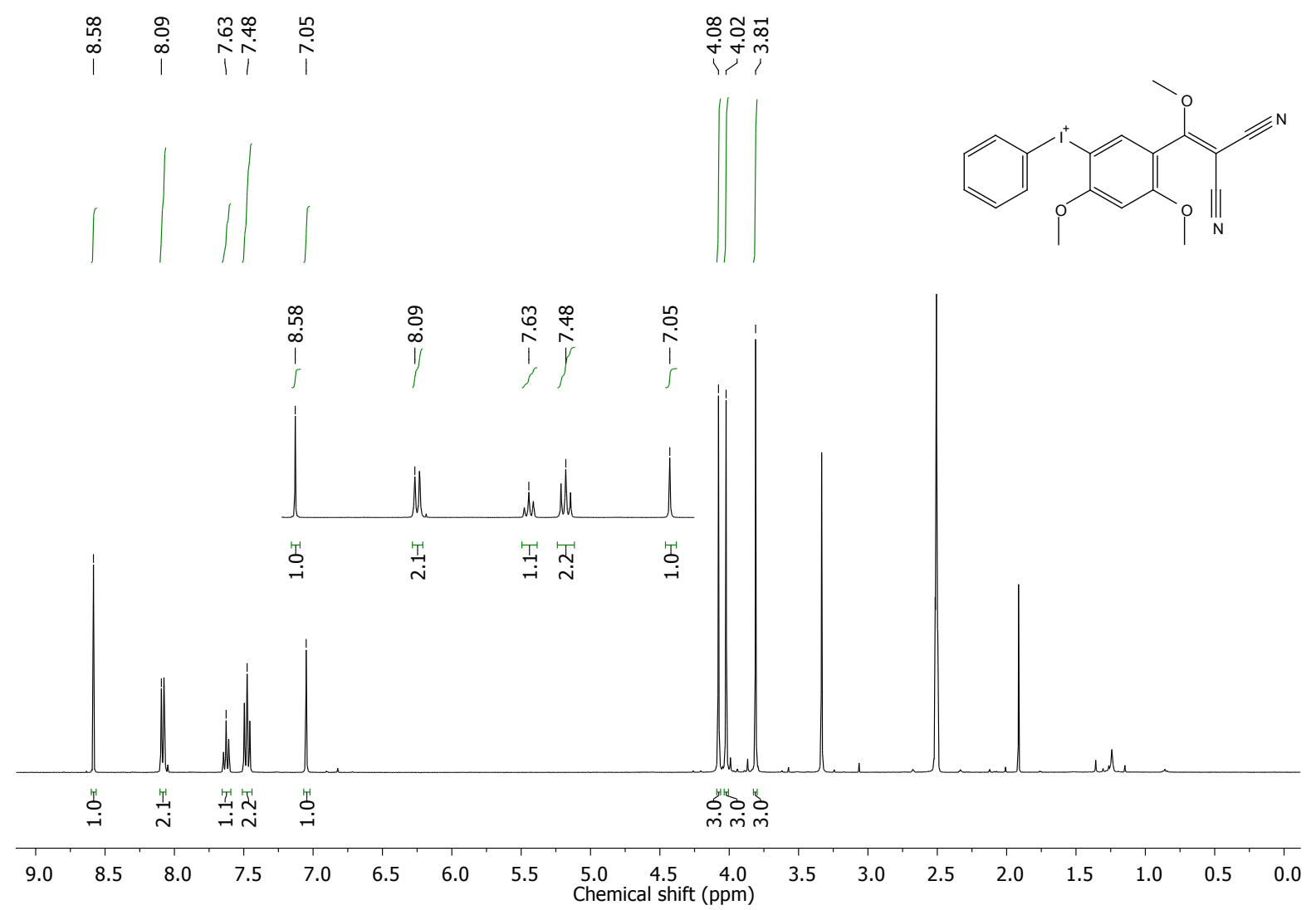

Figure S18. ${ }^{1} \mathrm{H}$ NMR of Comb-OMe-PI.

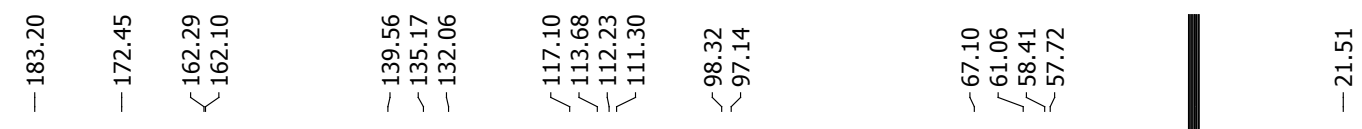

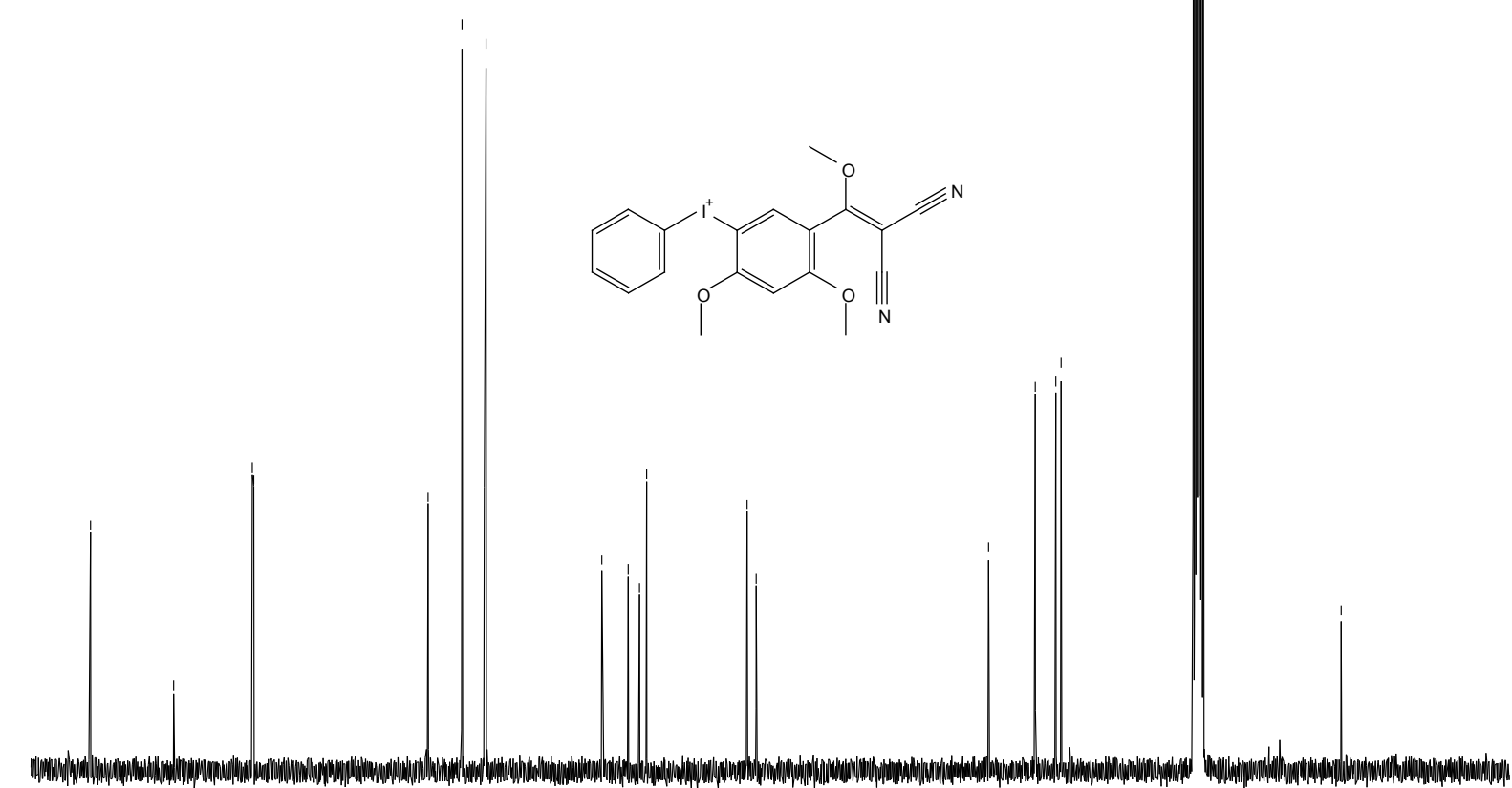

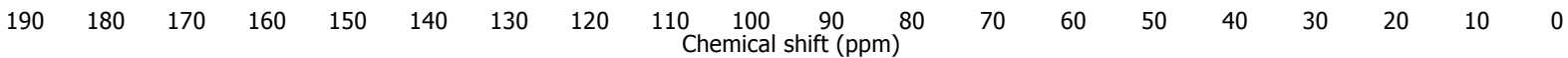

Figure S19. ${ }^{13} \mathrm{C}$ NMR of Comb-OMe-PI. 


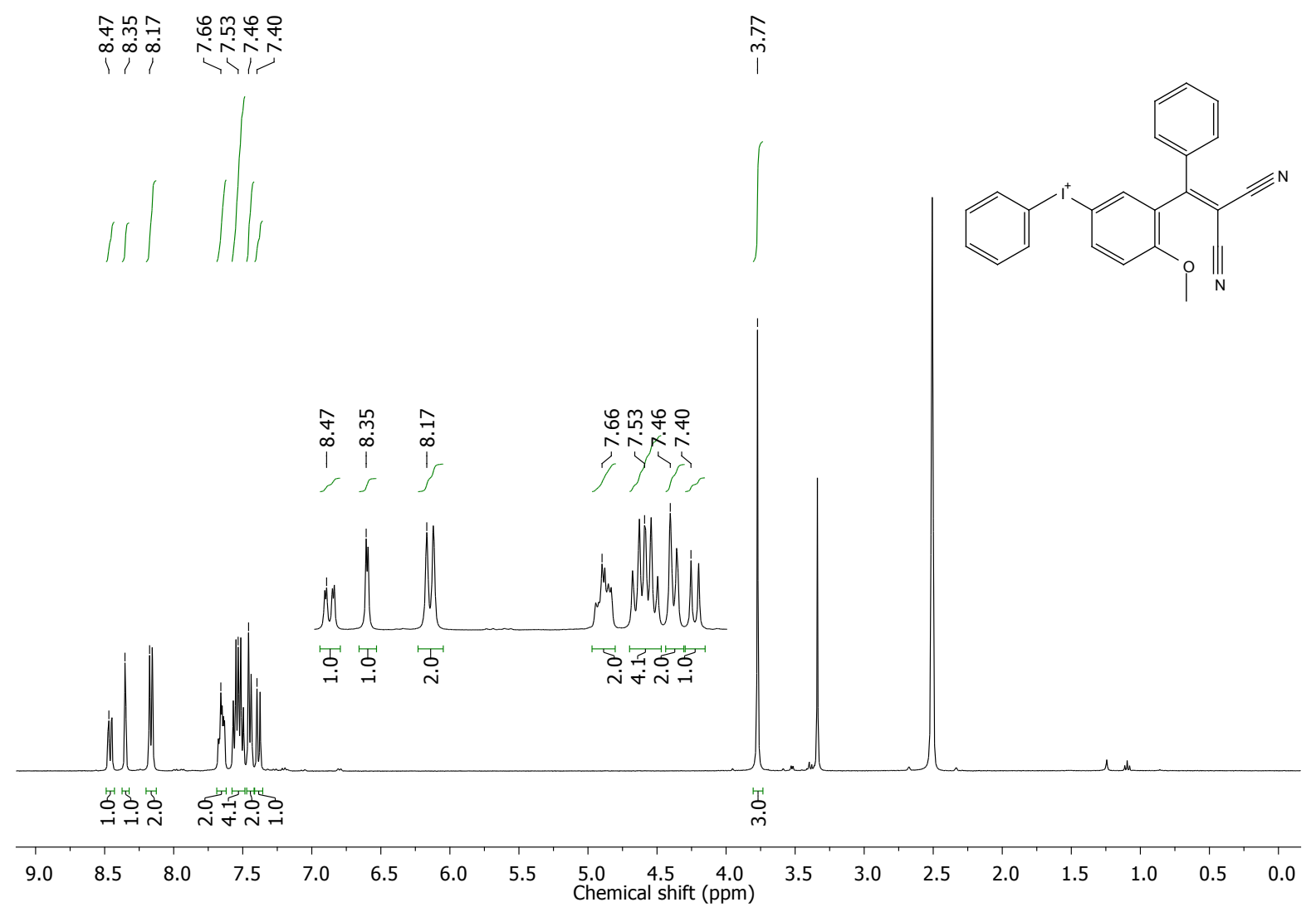

Figure S20. ${ }^{1} \mathrm{H}$ NMR of $\beta$-Ph-PI.
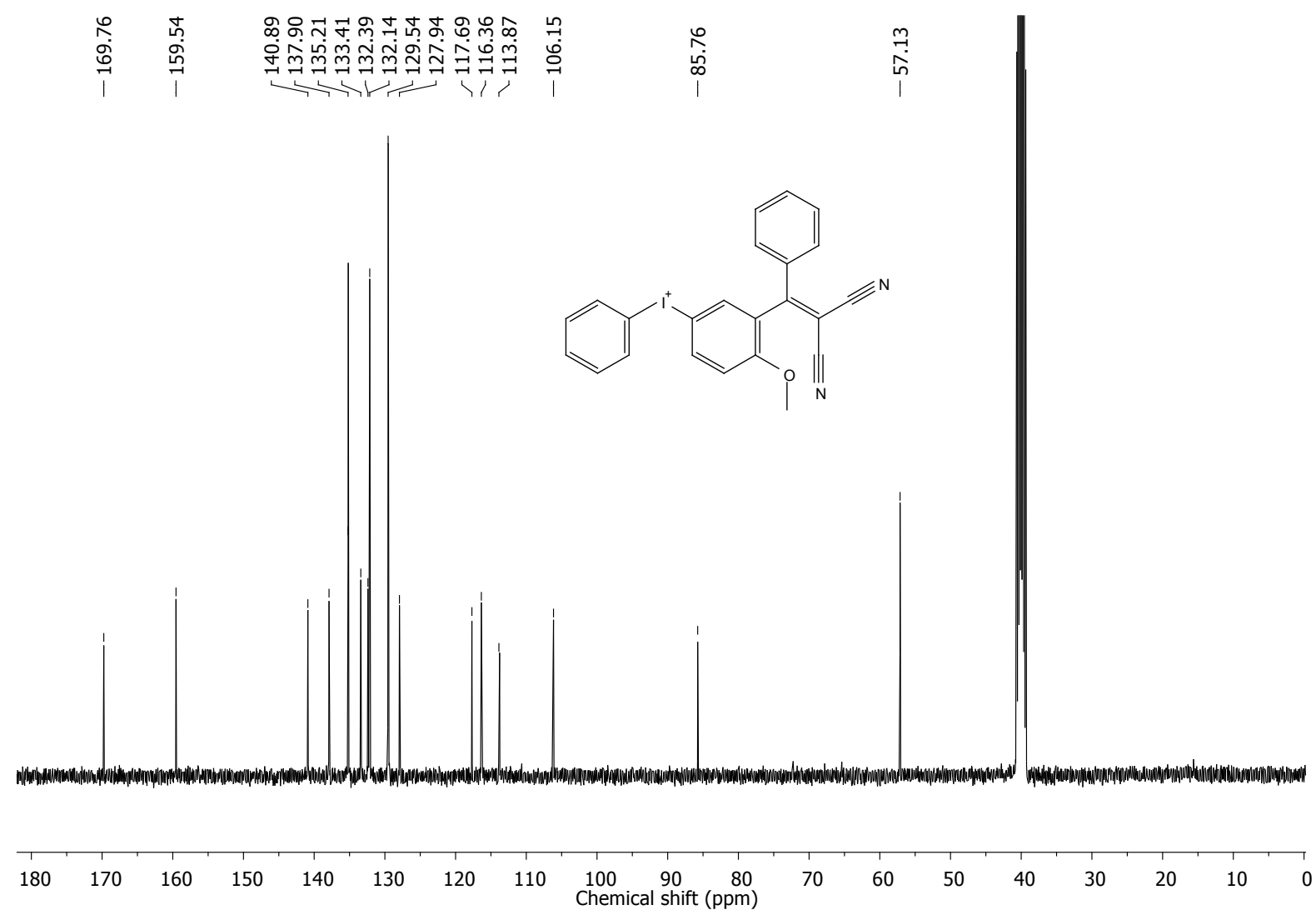

Figure S21. ${ }^{13} \mathrm{C}$ NMR of $\beta$-Ph-PI. 


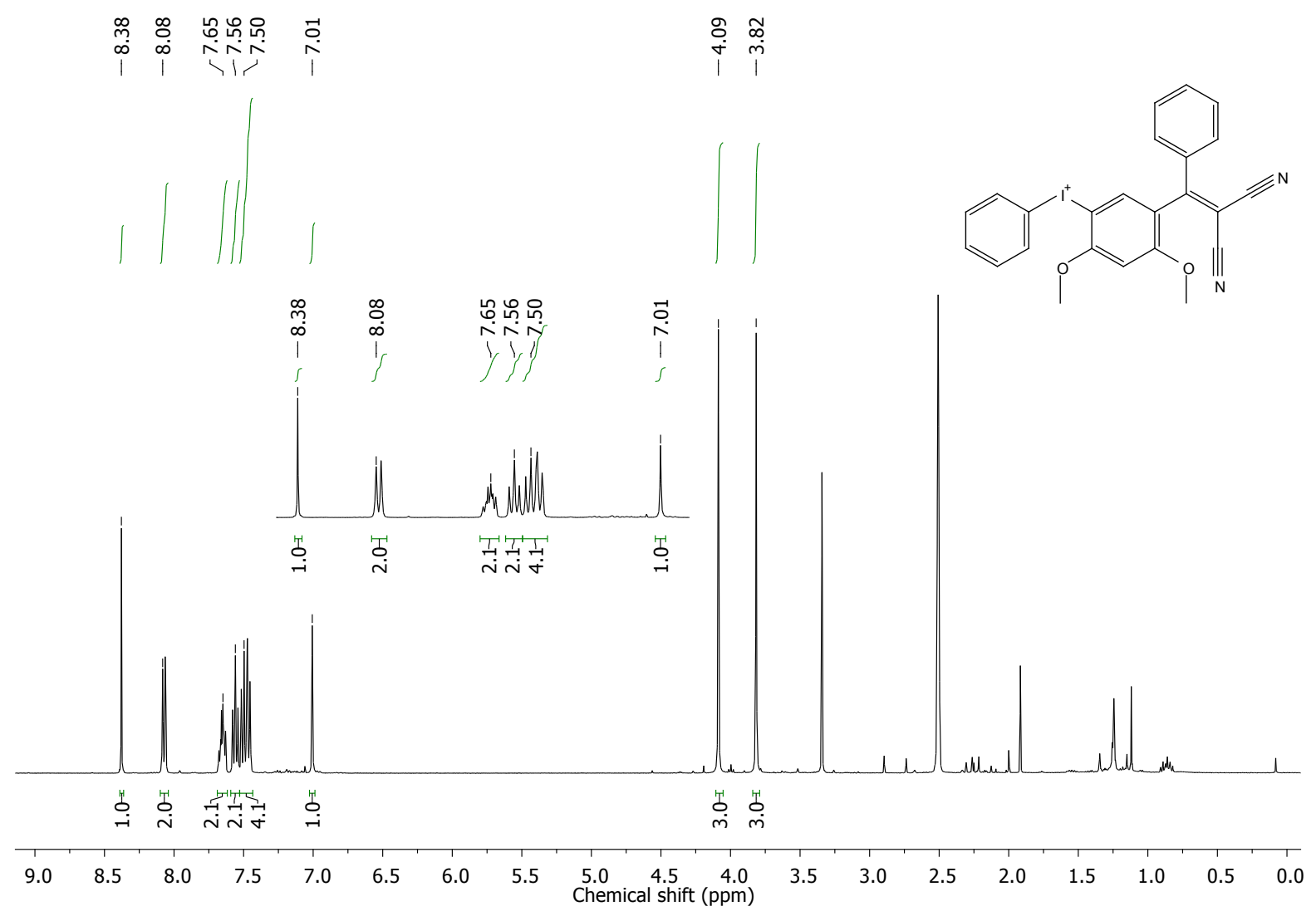

Figure S22. ${ }^{1 H}$ NMR of Comb-Ph-PI.

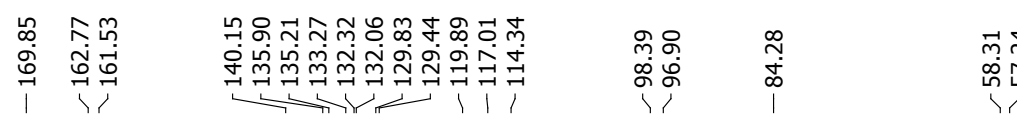

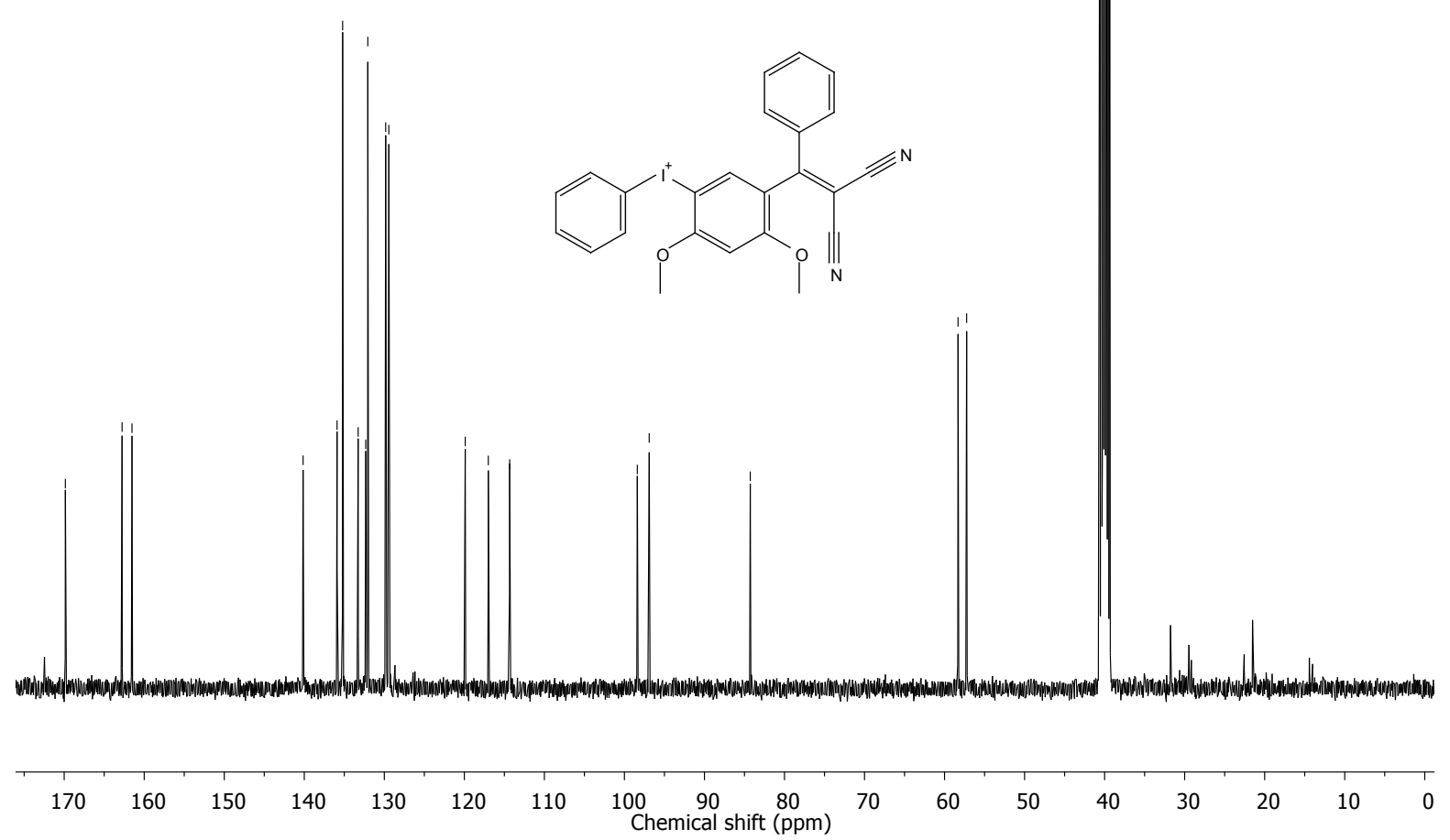

Figure S23. ${ }^{13} \mathrm{C}$ NMR of Comb-Ph-PI. 


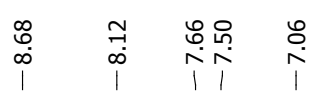

$$
\int 11
$$

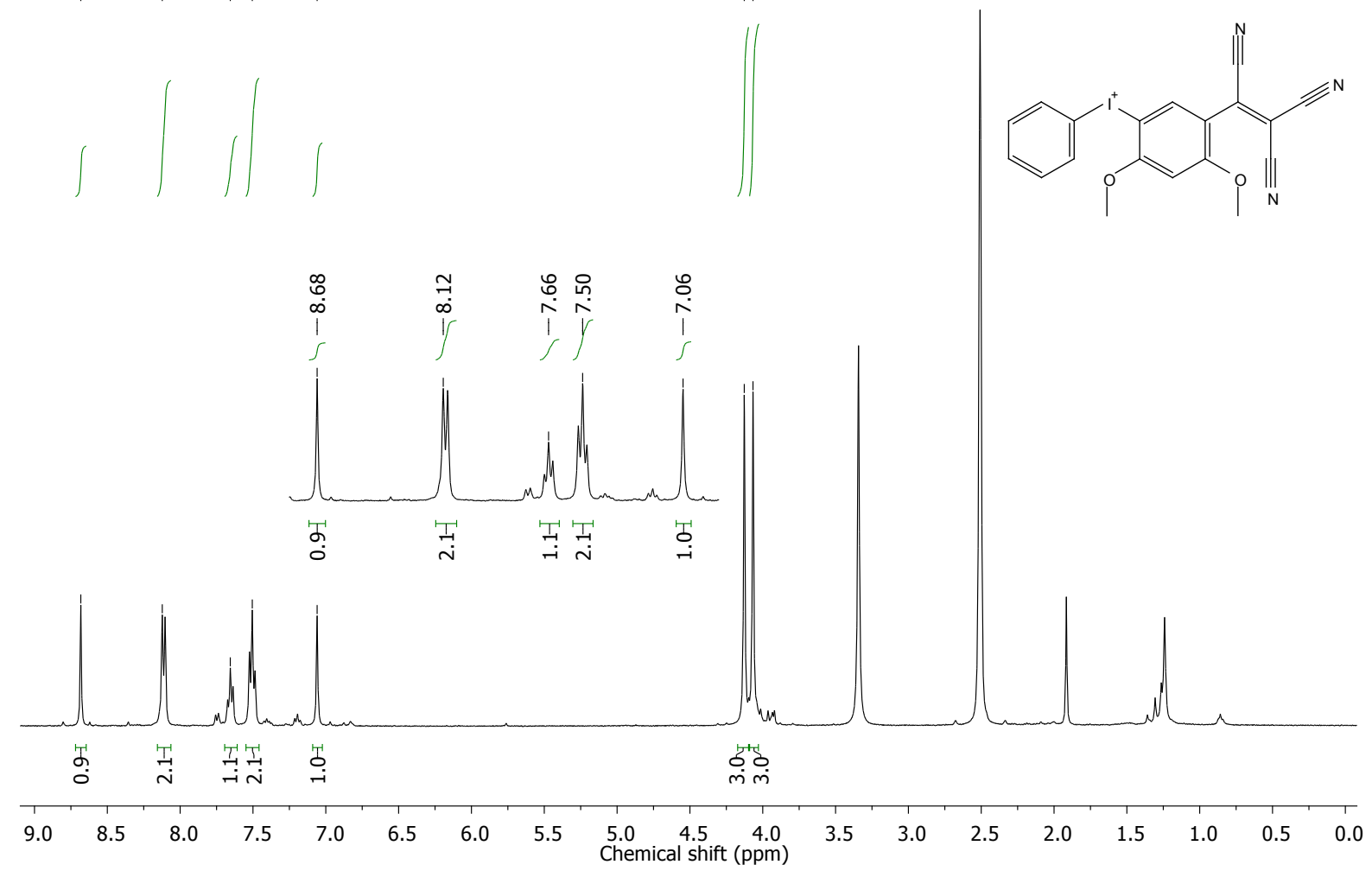

Figure S24. ${ }^{1} \mathrm{H}$ NMR of Comb-CN-PI. $m$
$\substack{7 \\ \dot{+}}$
$i$
$i$

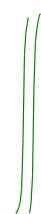

लें

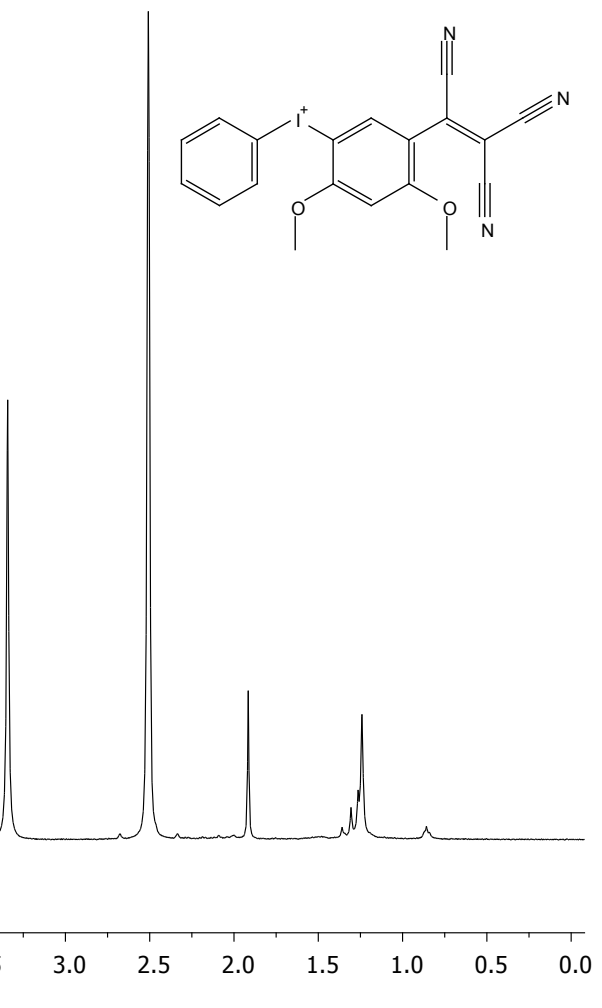

Figure S24. 1HNMR of Comb-CN-PI.
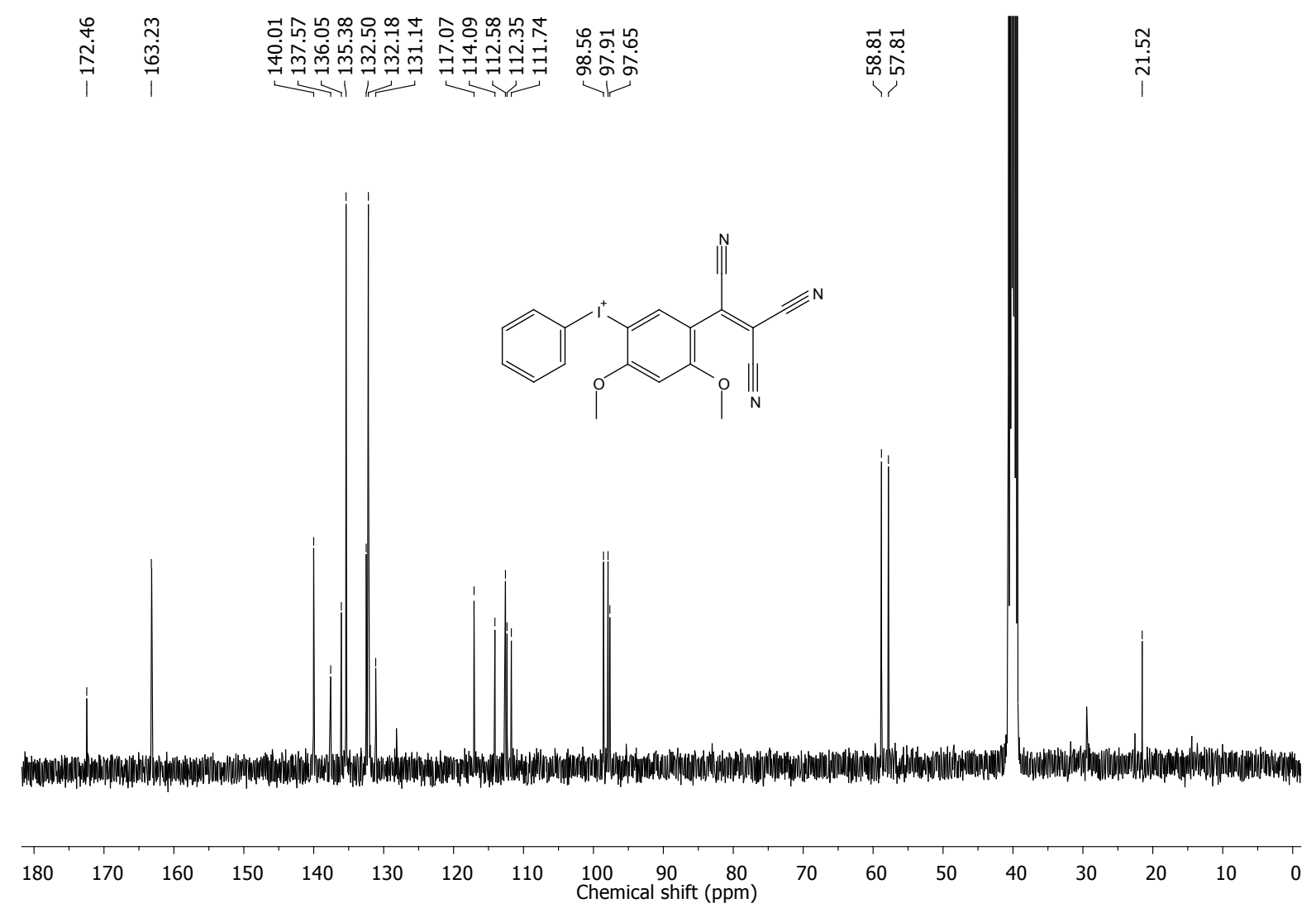

Figure S25. ${ }^{13} \mathrm{C}$ NMR of Comb-CN-PI 


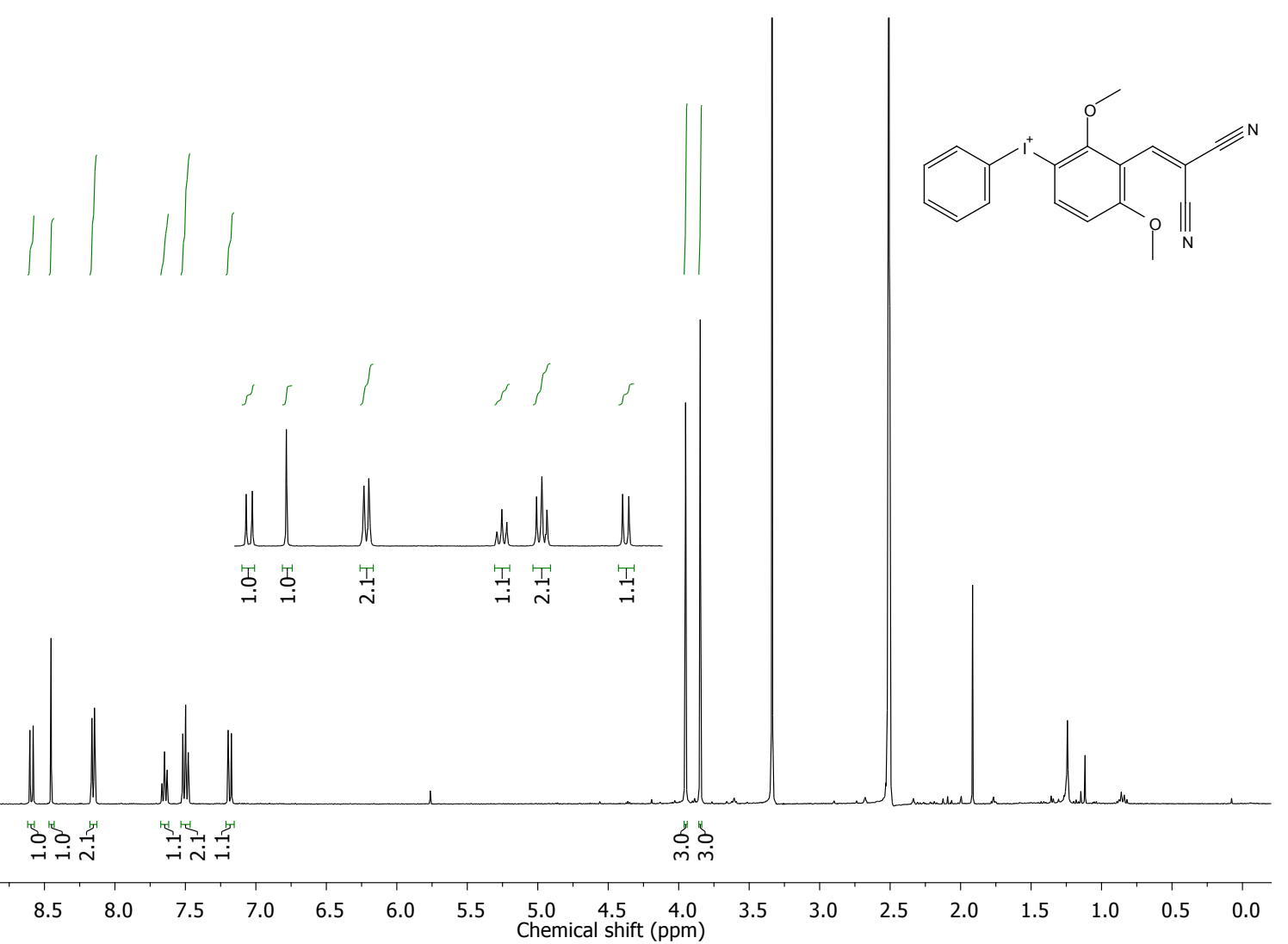

Figure S26. ${ }^{1} \mathrm{H}$ NMR of $A r-2,6-\mathrm{OMe}-\mathrm{Pl}$.

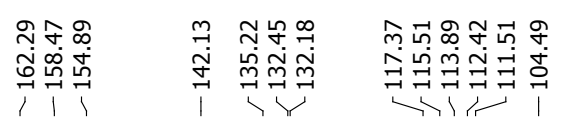

总

总紊
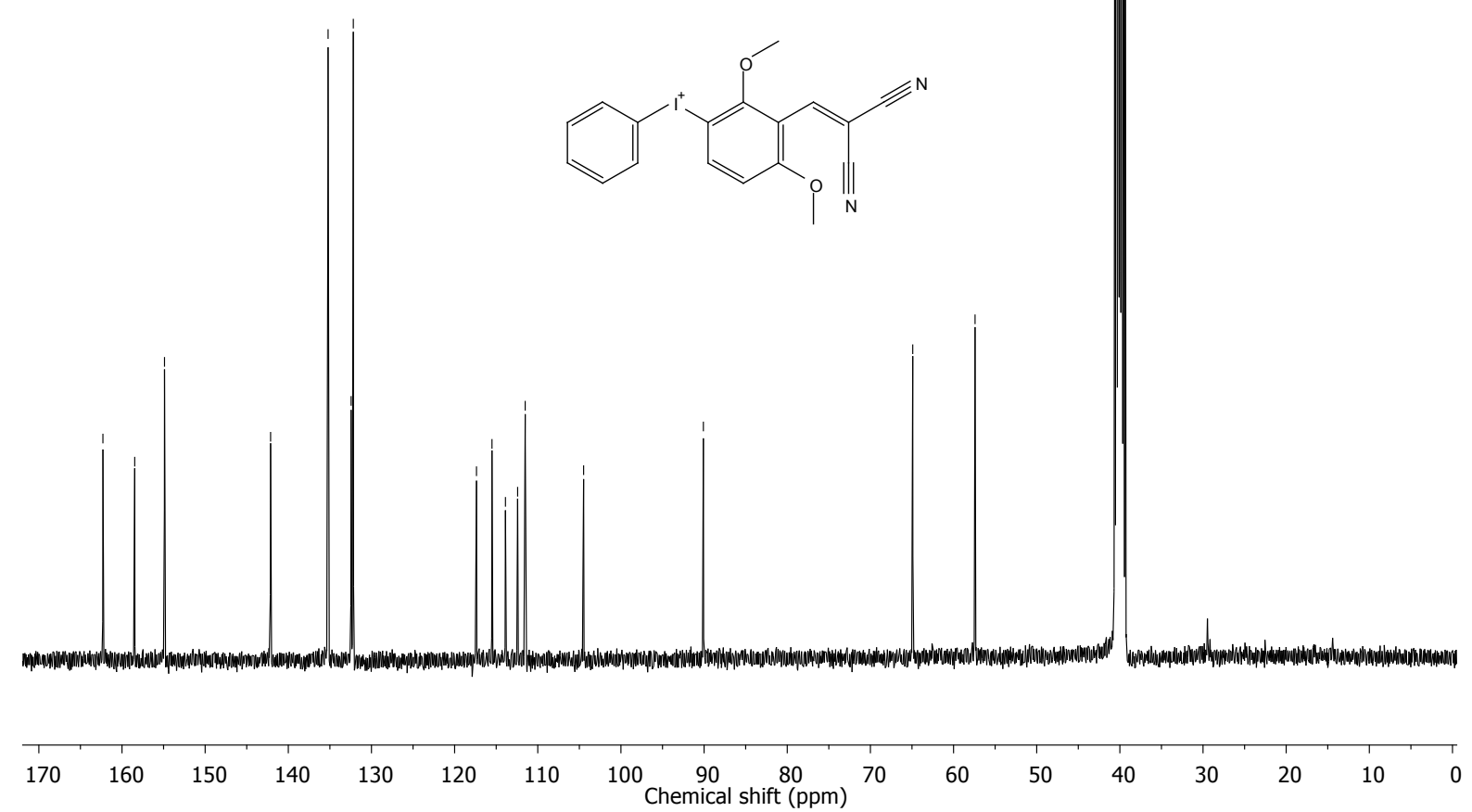

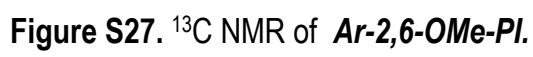




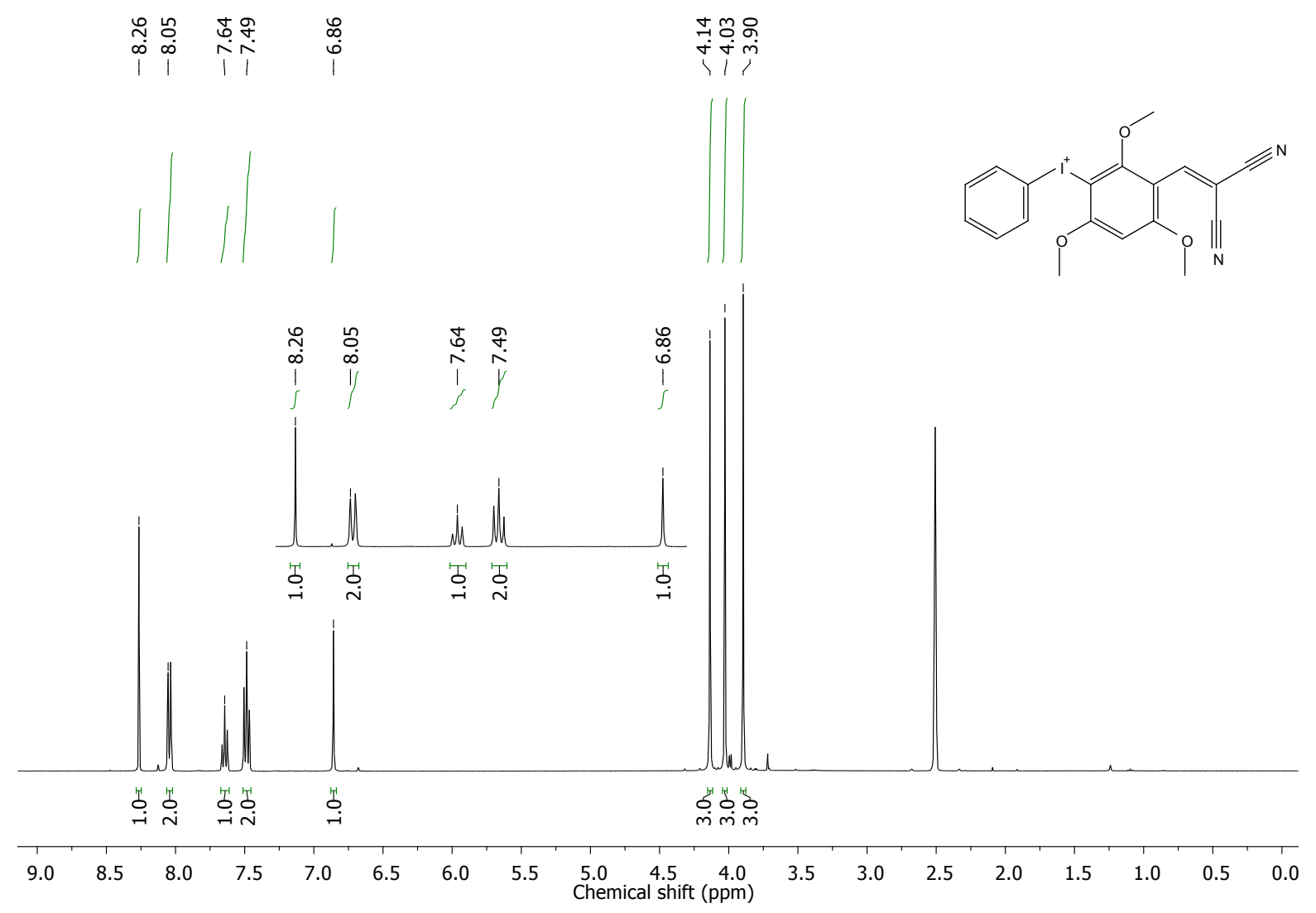

Figure S28. ${ }^{1} \mathrm{H}$ NMR of $A r-2,4,6-O M e-P I$.

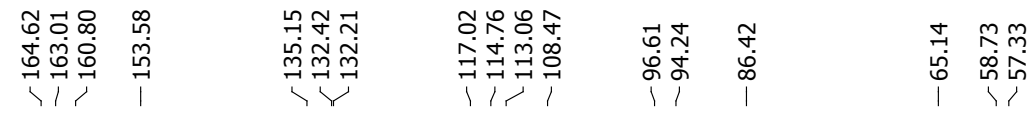
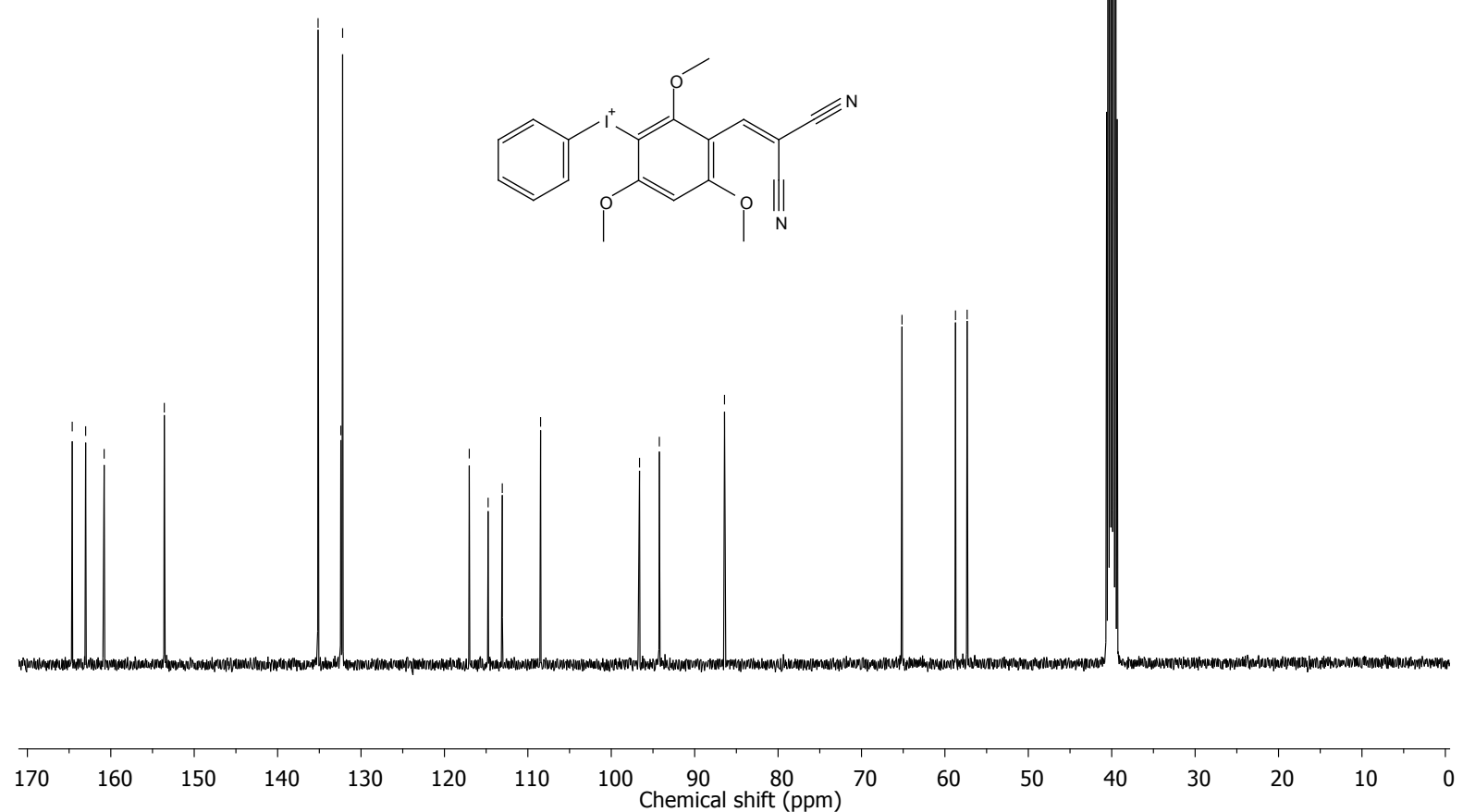

Figure S29. ${ }^{13} \mathrm{C}$ NMR of $\mathrm{Ar}-2,4,6-\mathrm{OMe}-\mathrm{Pl}$. 


\section{Section C. Photochemical experiments}

1. Comparison of absorption properties of iodonium salts with their chromophores.

Alpha position modified:

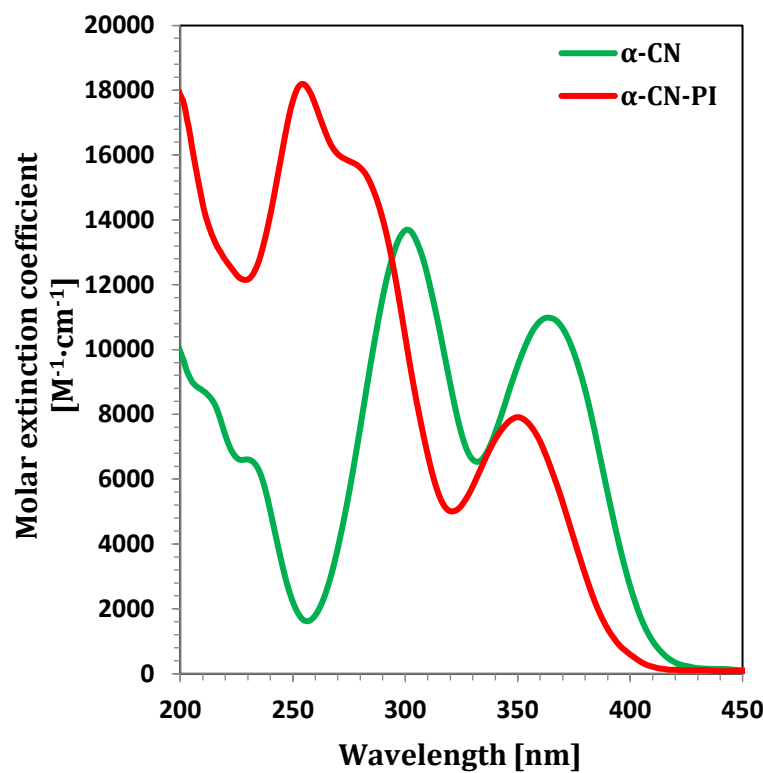

Figure S30. Comparison of UV-VIS absorption spectra of iodonium salt $\alpha-C N-P I$ and its chromophore $\alpha-C N$.

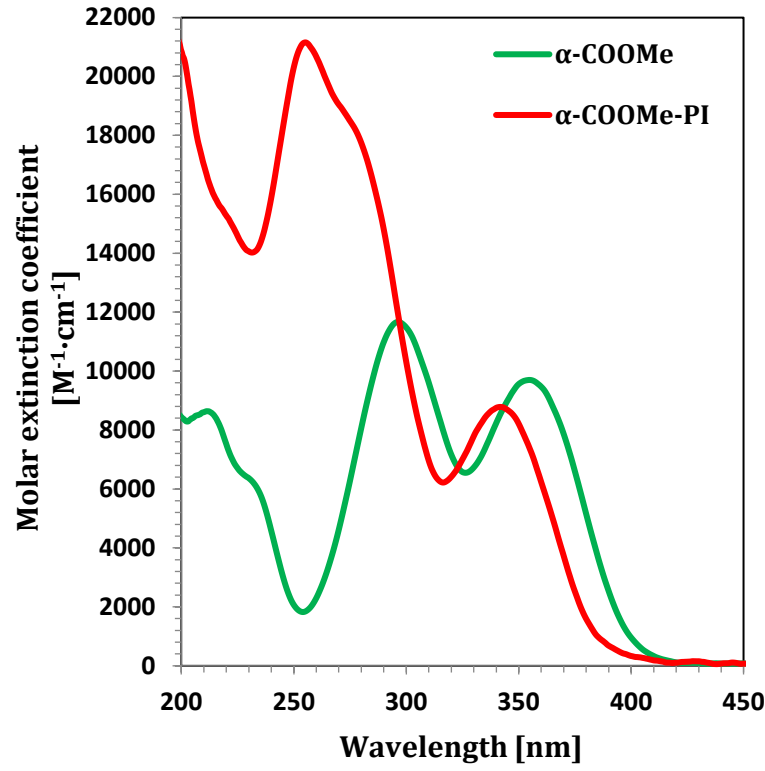

Figure S32. Comparison of UV-VIS absorption spectra of iodonium salt $\alpha-\mathrm{COOMe}-P I$ and its chromophore a-COOMe.

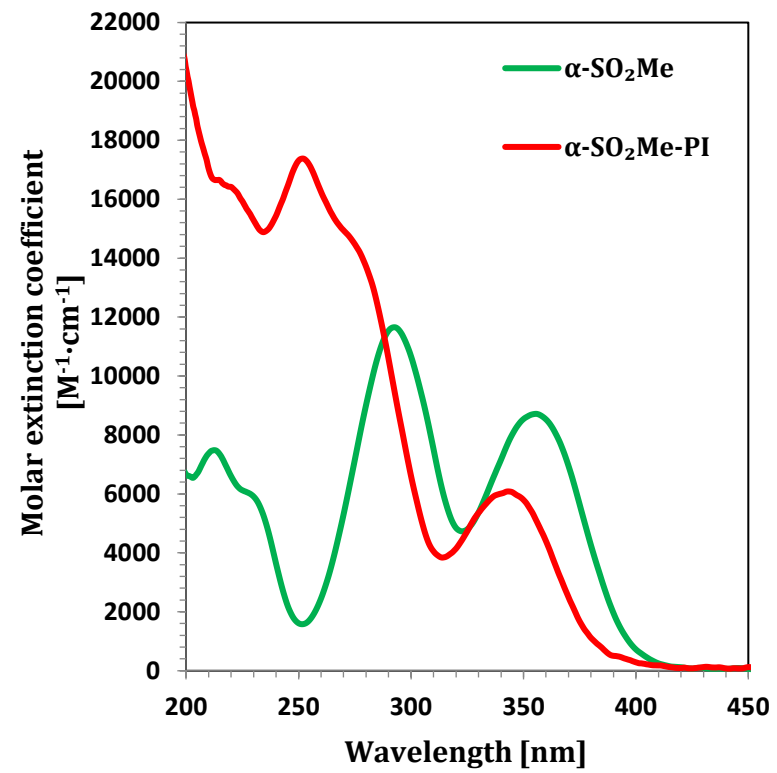

Figure S31. Comparison of UV-VIS absorption spectra of iodonium salt $\alpha-\mathrm{SO}_{2} \mathrm{Me}-\mathrm{PI}$ and its chromophore $\alpha-\mathrm{SO}_{2} \mathrm{Me}$.

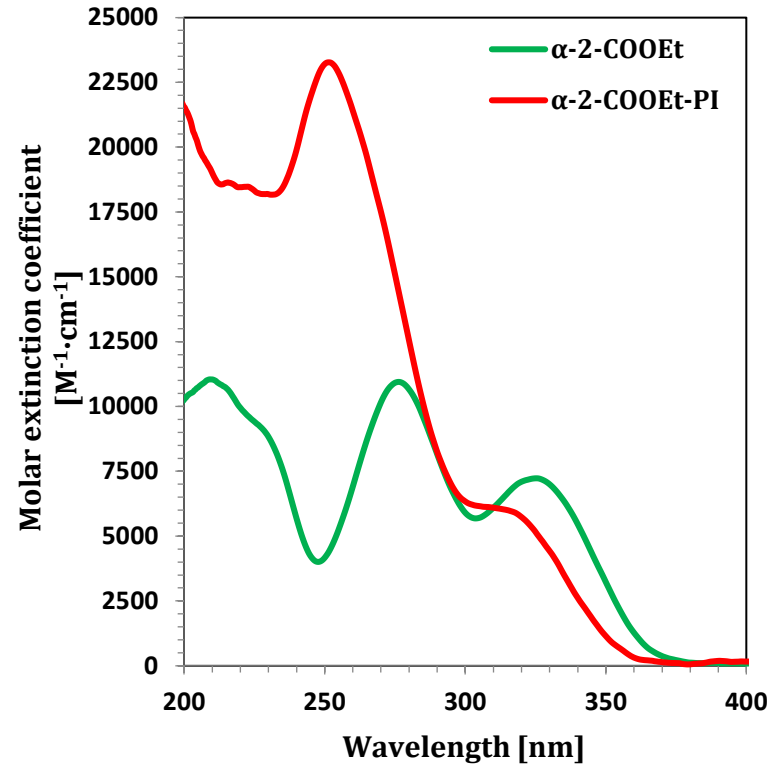

Figure S33. Comparison of UV-VIS absorption spectra of iodonium salt $\alpha-2-C O O E t-P I$ and its chromophore a-2-COOEt. 
Beta position modified:

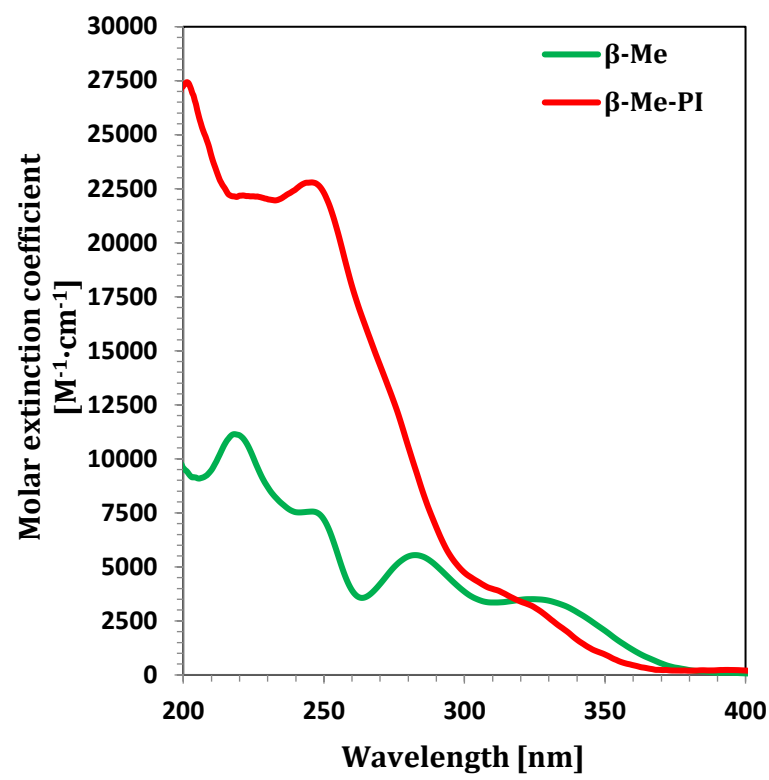

Figure S34. Comparison of UV-VIS absorption spectra of iodonium salt $\beta-M e-P I$ and its chromophore $\beta-M e$.

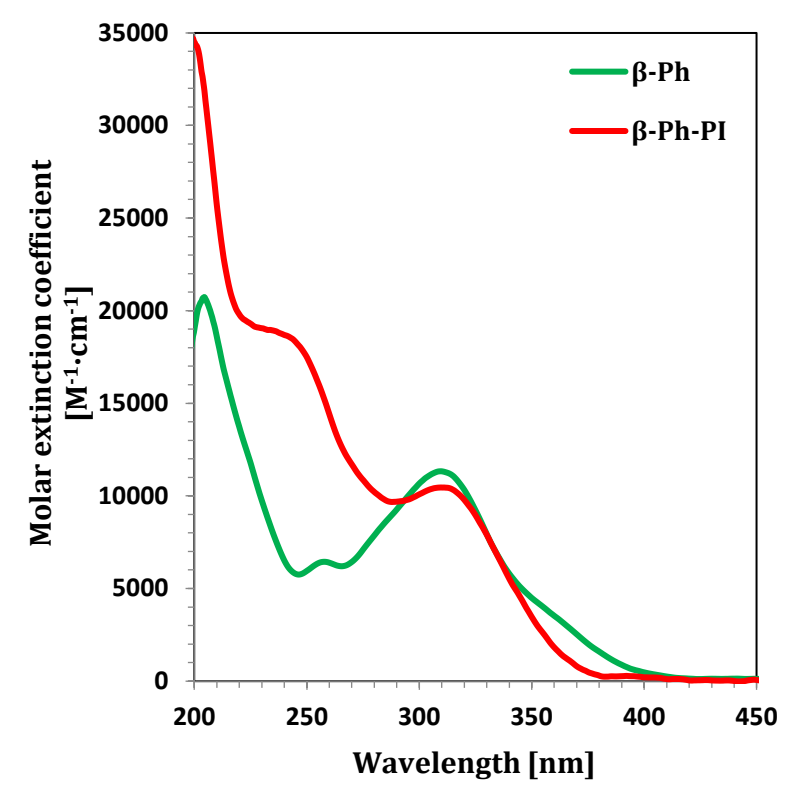

Figure S36. Comparison of UV-VIS absorption spectra of iodonium salt $\beta-P h-P I$ and its chromophore $\beta-P h$.

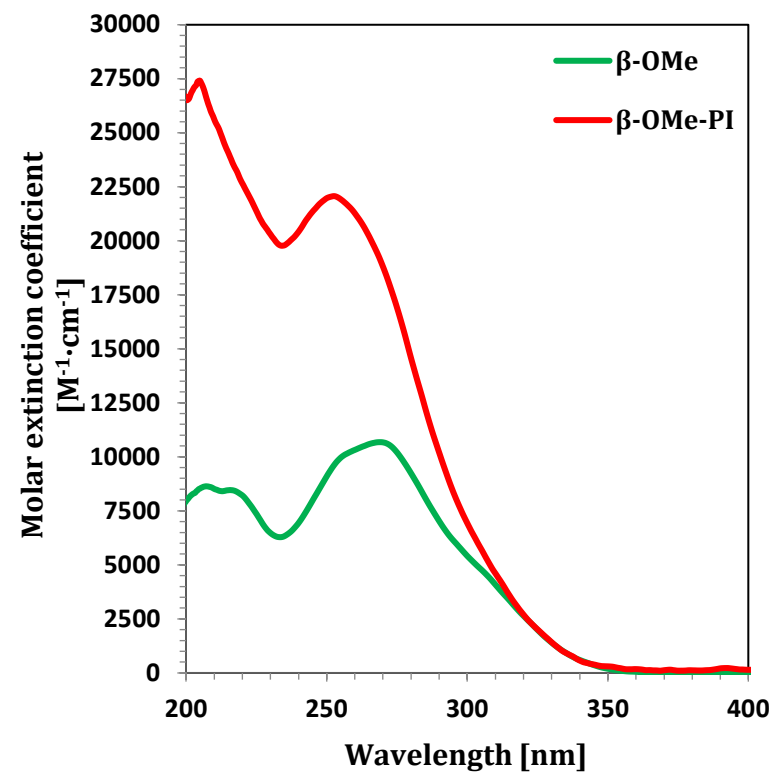

Figure S35. Comparison of UV-VIS absorption spectra of iodonium salt $\beta-O M e-P I$ and its chromophore $\beta-O M e$. 
Aryl ring modified:

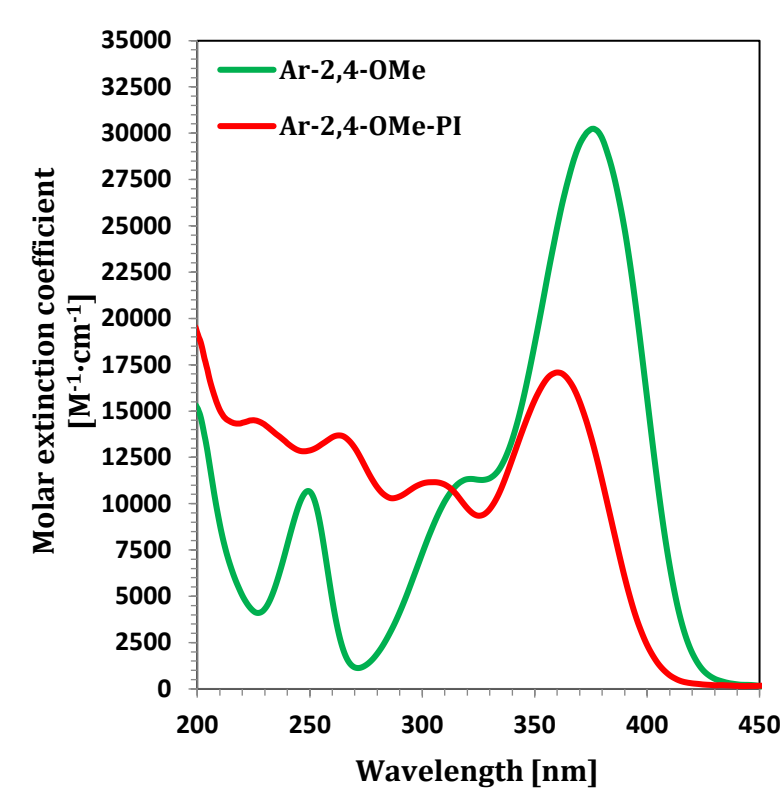

Figure S37. Comparison of UV-VIS absorption spectra of iodonium salt Ar-2,4-OMe-PI and its chromophore Ar-2,4-OMe.

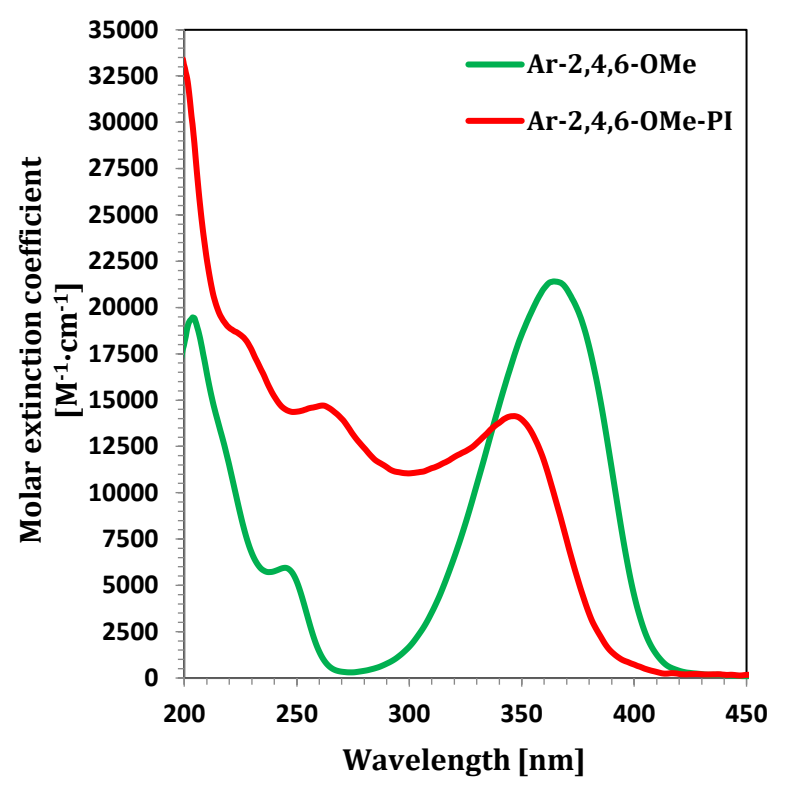

Figure S39. Comparison of UV-VIS absorption spectra of iodonium salt $\mathrm{Ar}-2,4,6-\mathrm{OMe}-\mathrm{PI}$ and its chromophore Ar-2,4,6-OMe.

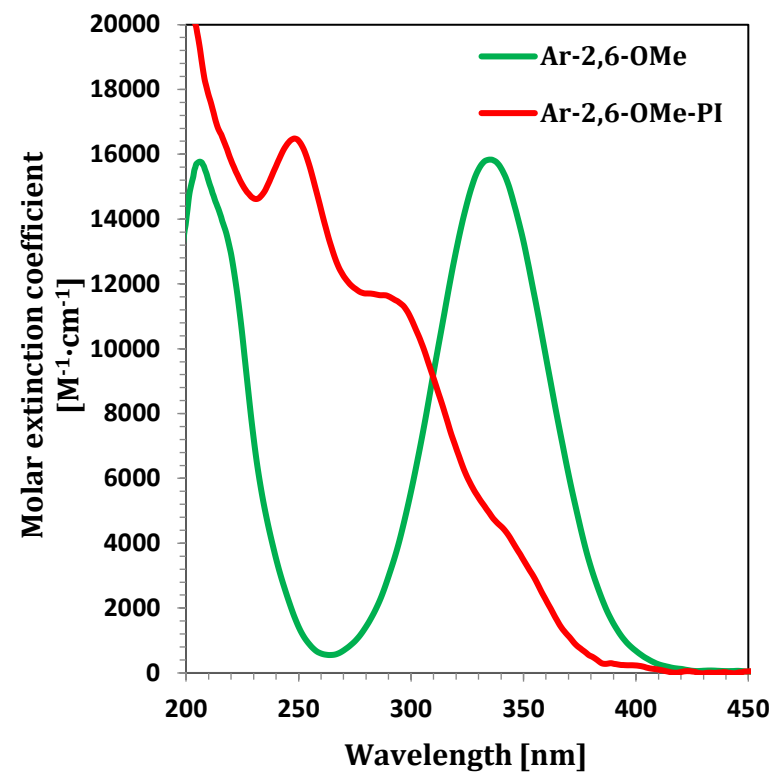

Figure S38. Comparison of UV-VIS absorption spectra of iodonium salt $\mathrm{Ar}-2,6-\mathrm{OMe}-\mathrm{PI}$ and its chromophore Ar-2,6-OMe. 
Combined modifications:

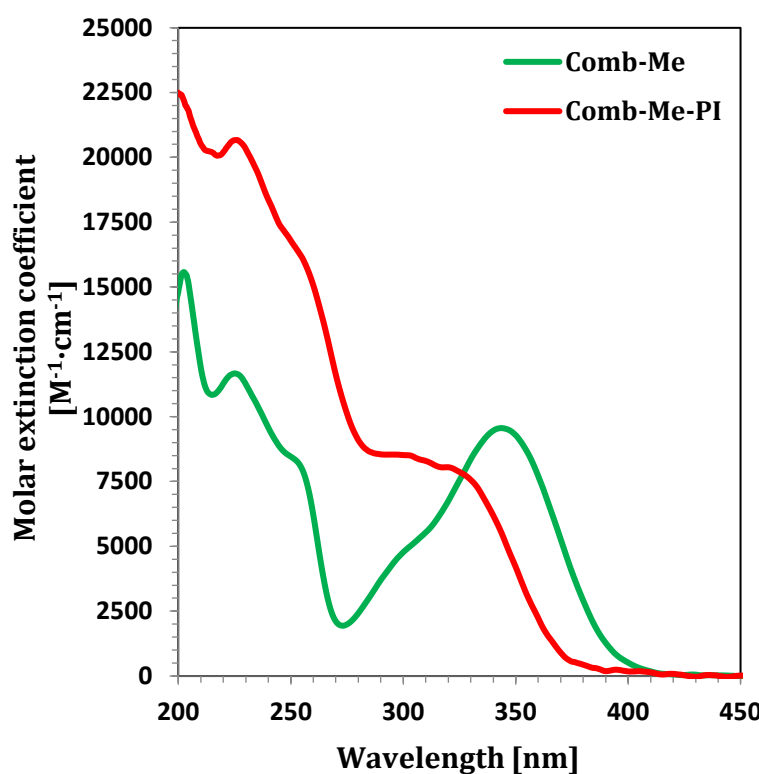

Figure S40. Comparison of UV-VIS absorption spectra of iodonium salt Comb-Me-PI and its chromophore Comb-Me.

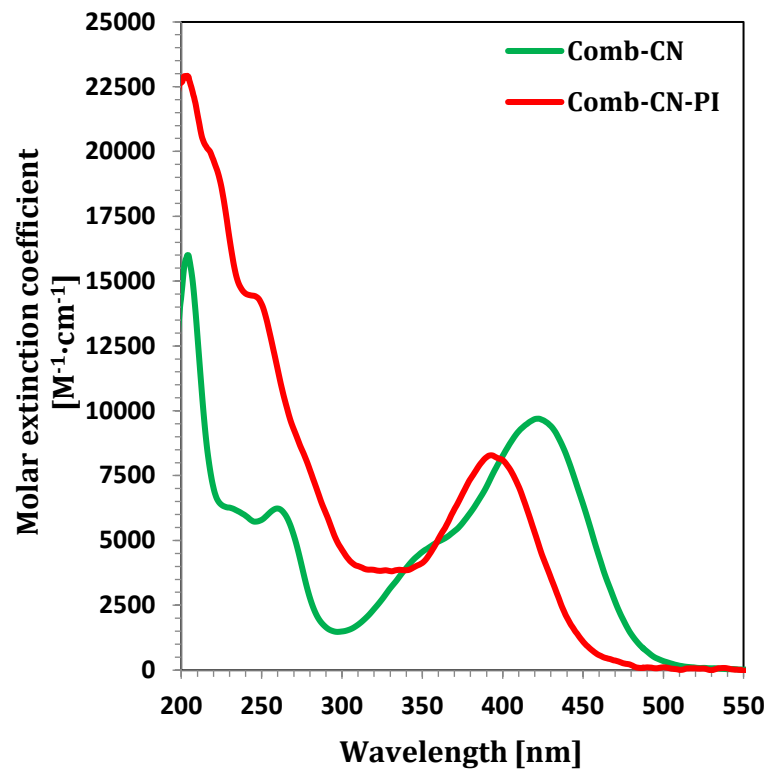

Figure S42. Comparison of UV-VIS absorption spectra of iodonium salt Comb-CN-PI and its chromophore Comb-CN.

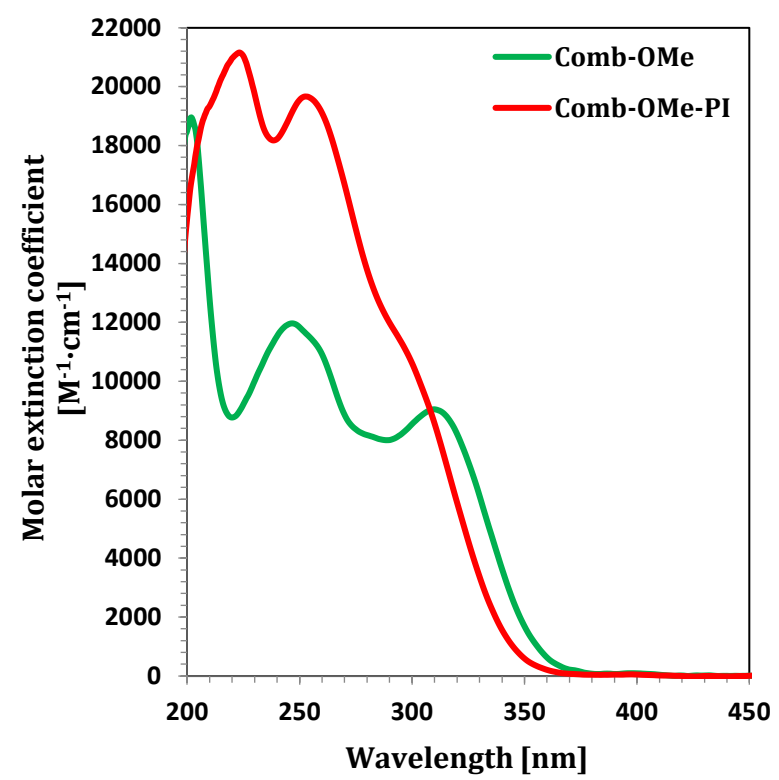

Figure S41. Comparison of UV-VIS absorption spectra of iodonium salt Comb-OMe-PI and its chromophore Comb-OMe.

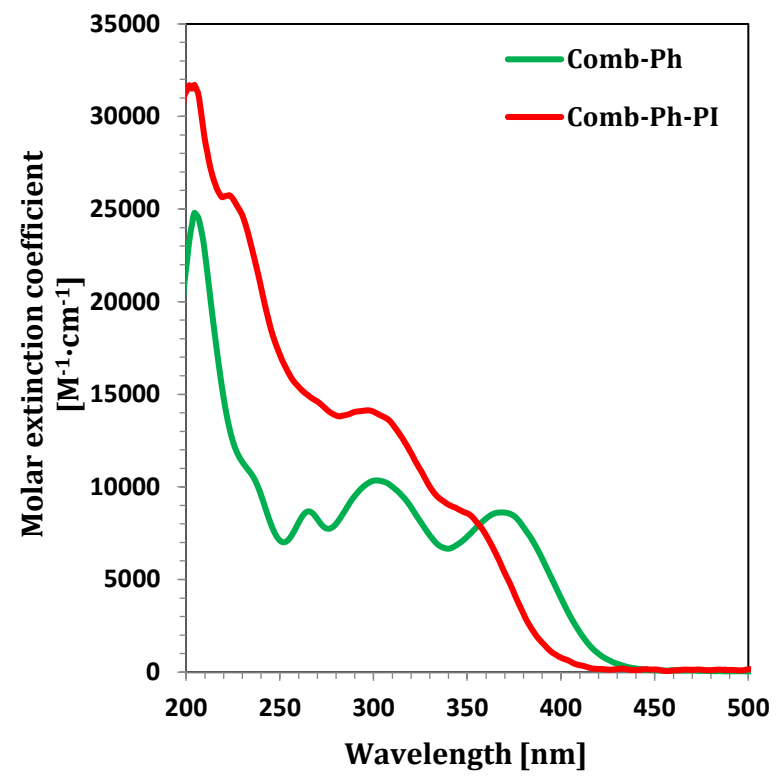

Figure S43. Comparison of UV-VIS absorption spectra of iodonium salt Comb-Ph-PI and its chromophore Comb-Ph 
Table S1. Comparison of spectral properties of chromophores and their iodonium salts in terms of absorption bands positions.

\begin{tabular}{|c|c|c|c|}
\hline \multicolumn{4}{|c|}{ a-position modified chromophores and their iodonium salts } \\
\hline Compund & $\begin{array}{c}\lambda_{\text {max-ab }} \\
{[\mathrm{nm}]}\end{array}$ & $\begin{array}{l}\Delta \lambda_{\max } \\
{[\mathrm{nm}]}\end{array}$ & Type of absorption shift \\
\hline$\alpha-C N$ & 363 & \multirow{2}{*}{13} & \multirow{2}{*}{ Hypsochromic shift } \\
\hline$\alpha-C N-P I$ & 350 & & \\
\hline$\alpha-\mathrm{SO}_{2} \mathrm{Me}$ & 355 & \multirow{2}{*}{12} & \multirow{2}{*}{ Hypsochromic shift } \\
\hline$\alpha-\mathrm{SO}_{2} \mathrm{Me}-\mathrm{PI}$ & 343 & & \\
\hline$\alpha-C O O M e$ & 354 & \multirow{2}{*}{12} & \multirow{2}{*}{ Hypsochromic shift } \\
\hline$\alpha-C O O M e-P I$ & 342 & & \\
\hline a-2-COOEt & 325 & \multirow{2}{*}{17} & \multirow{2}{*}{ Hypsochromic shift } \\
\hline$\alpha-2-C O O E t-P I$ & 308 & & \\
\hline \multicolumn{4}{|c|}{ B-position modified chromospheres and their iodonium salts } \\
\hline Compund & $\begin{array}{c}\lambda_{\text {max-ab }} \\
{[\mathrm{nm}]}\end{array}$ & $\begin{array}{c}\Delta \lambda_{\max } \\
{[\mathrm{nm}]}\end{array}$ & Type of absorption shift \\
\hline$\beta$-Me & 324 & \multirow{2}{*}{12} & \multirow{2}{*}{ Hypsochromic shift } \\
\hline$\beta-M e-P I$ & 312 & & \\
\hline$\beta-O M e$ & 269 & \multirow{2}{*}{17} & \multirow{2}{*}{ Hypsochromic shift } \\
\hline$\beta-O M e-P I$ & 252 & & \\
\hline$\beta-P h$ & 309 & \multirow{2}{*}{0} & \multirow{2}{*}{ No shift } \\
\hline$\beta-P h-P I$ & 309 & & \\
\hline \multicolumn{4}{|c|}{ Aryl modified chromospheres and their iodonium salts } \\
\hline Compund & $\begin{array}{c}\lambda_{\text {max-ab }} \\
{[\mathrm{nm}]}\end{array}$ & $\begin{array}{c}\Delta \lambda_{\max } \\
{[\mathrm{nm}]}\end{array}$ & Type of absorption shift \\
\hline$A r-2,4-O M e$ & 376 & \multirow{2}{*}{16} & \multirow{2}{*}{ Hypsochromic shift } \\
\hline Ar-2,4-OMe-PI & 360 & & \\
\hline$A r-2,6-O M e$ & 335 & \multirow{2}{*}{40} & \multirow{2}{*}{ Hypsochromic shift } \\
\hline Ar-2,6-OMe-PI & 295 & & \\
\hline$A r-2,4,6-O M e$ & 364 & \multirow{2}{*}{17} & \multirow{2}{*}{ Hypsochromic shift } \\
\hline$A r-2,4,6-O M e-P I$ & 347 & & \\
\hline \multicolumn{4}{|c|}{ Combined modified derivatives of chromospheres and their iodonium salts } \\
\hline Compund & $\begin{array}{c}\lambda_{\text {max-ab }} \\
{[\mathrm{nm}]}\end{array}$ & $\begin{array}{c}\Delta \lambda_{\max } \\
{[\mathrm{nm}]}\end{array}$ & Type of absorption shift \\
\hline Comb-Me & 343 & \multirow{2}{*}{38} & \multirow{2}{*}{ Hypsochromic shift } \\
\hline Comb-Me-PI & 305 & & \\
\hline Comb-OMe & 310 & \multirow{2}{*}{57} & \multirow{2}{*}{ Hypsochromic shift } \\
\hline Comb-OMe-PI & 253 & & \\
\hline Comb-CN & 422 & \multirow{2}{*}{30} & Hvysochromic shift \\
\hline Comb-CN-PI & 392 & & Hypsochromic shitt \\
\hline Comb-Ph & 368 & & \\
\hline Comb-Ph-PI & 297 & 71 & Hypsochromic shift \\
\hline
\end{tabular}


2. Absorption spectra of chromophores which cannnot couple to iodonium salts with used method.

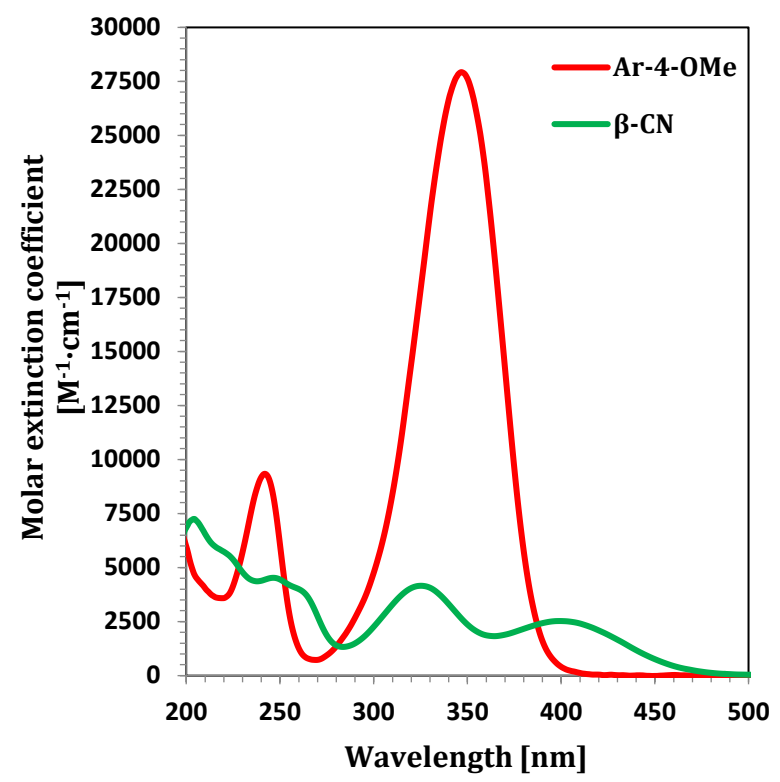

Figure S44. UV-VIS absorption spectra of chromophores which cannot couple to iodonium salt with used method.

3. Steady state photolysis experiments for chromophores

Alpha modified derivatives:

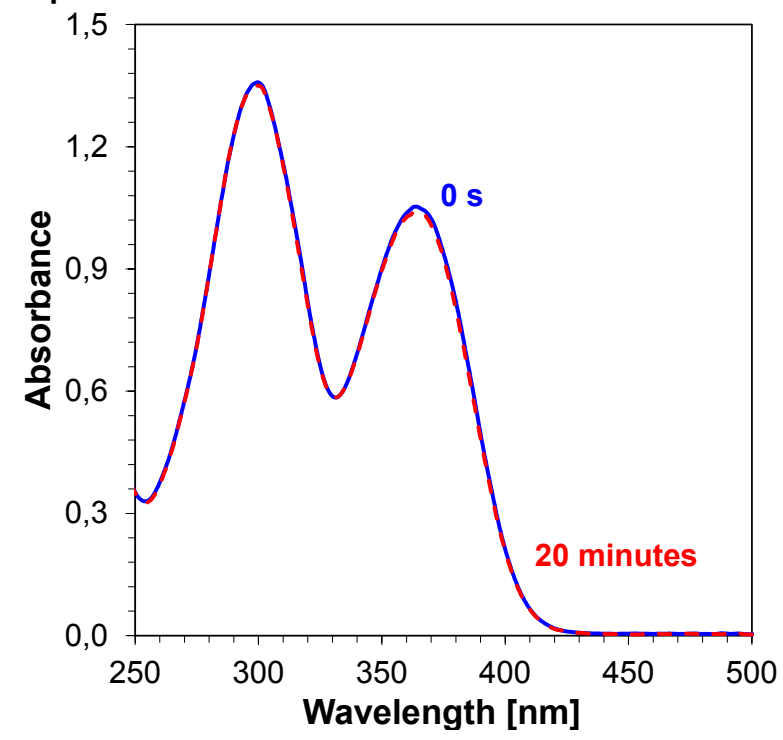

Figure S45. Photolysis of $\alpha-C N$ in acetonitrile upon exposure to LED at $365 \mathrm{~nm}\left(190 \mathrm{~mW} \cdot \mathrm{cm}^{-2}\right)$, concentration $[\alpha-C N]=1,60 \cdot 10^{-4} \mathrm{~mol}^{-} \cdot \mathrm{dm}^{-3}$.

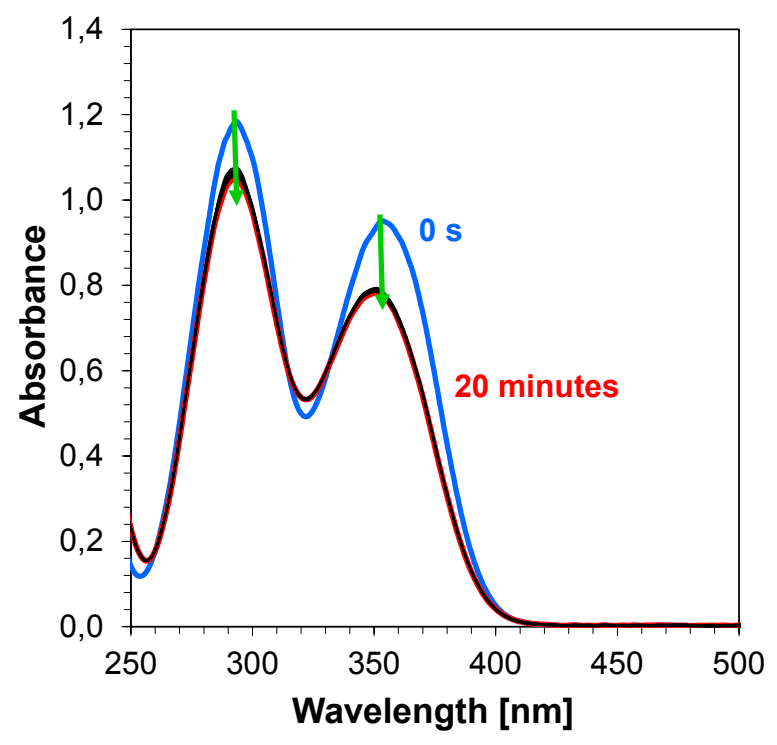

Figure S46. Photolysis of $\alpha-\mathrm{SO}_{2} \mathrm{Me}$ in acetonitrile upon exposure to LED at $365 \mathrm{~nm}\left(190 \mathrm{~mW} \cdot \mathrm{cm}^{-2}\right)$, concentration $\left[a-\mathrm{SO}_{2} \mathrm{Me}\right]=9,79 \cdot 10^{-5} \mathrm{~mol}^{-\mathrm{dm}^{-3}}$. 


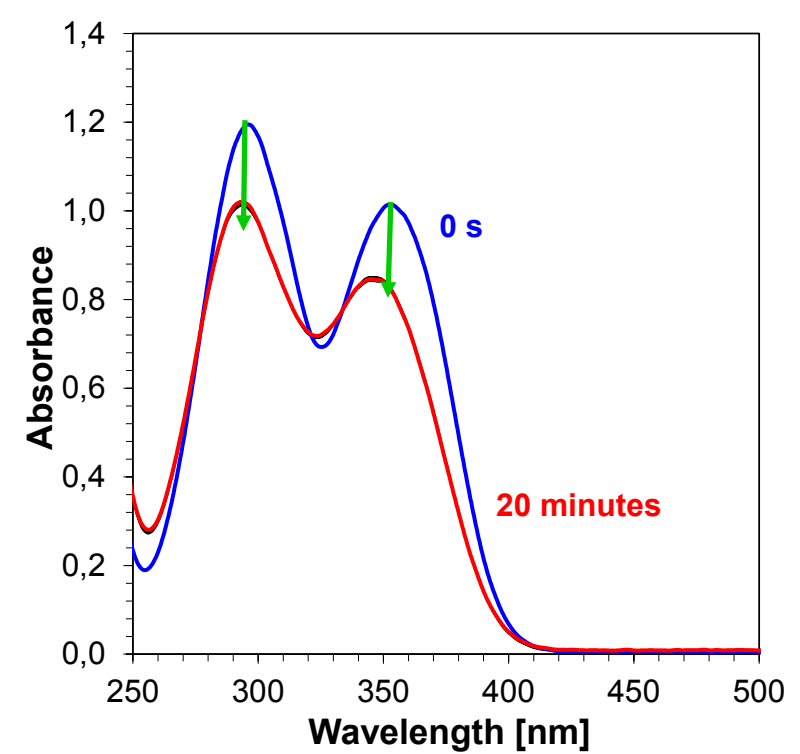

Figure S47. Photolysis of $\alpha-\mathrm{COOMe}$ in acetonitrile upon exposure to LED at $365 \mathrm{~nm}\left(190 \mathrm{~mW} \cdot \mathrm{cm}^{-2}\right)$, concentration $[\alpha-C O O M e]=1,17 \cdot 10^{-4} \mathrm{~mol} \cdot \mathrm{dm}^{-3}$.

Beta modified derivatives :

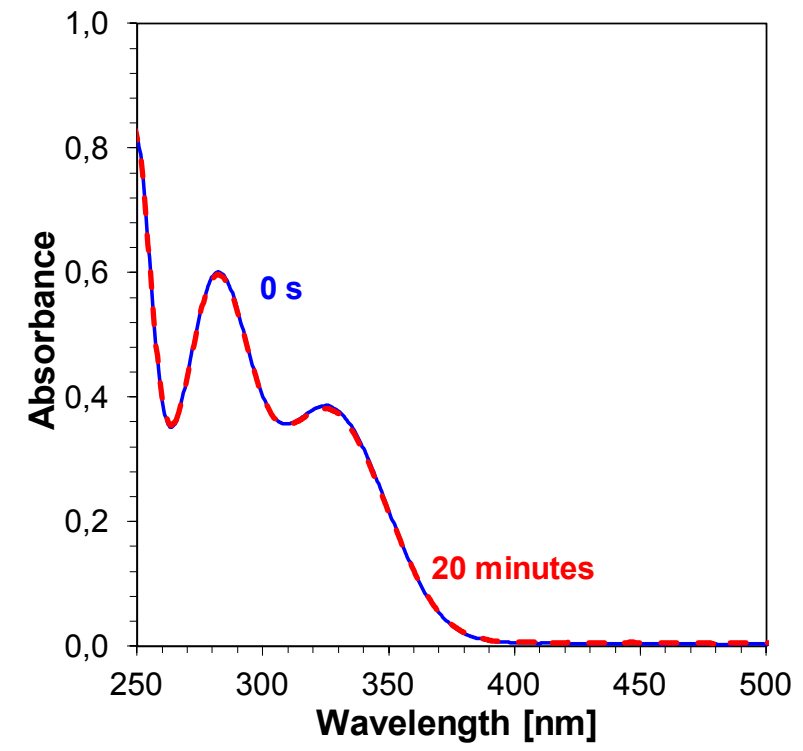

Figure S49. Photolysis of $\boldsymbol{\beta}-\mathbf{M e}$ in acetonitrile upon exposure to LED at $365 \mathrm{~nm}\left(190 \mathrm{~mW} \cdot \mathrm{cm}^{-2}\right)$, concentration $[\beta-M e]=9,49 \cdot 10^{-5} \mathrm{~mol} \cdot \mathrm{dm}^{-3}$.

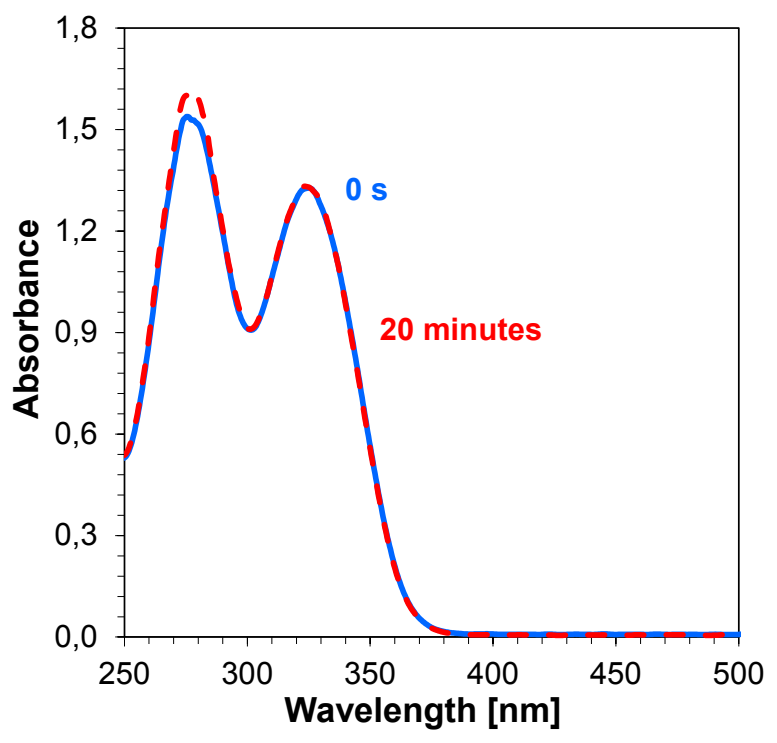

Figure S48. Photolysis of $\alpha-2-C O O E t$ in acetonitrile upon exposure to LED at $365 \mathrm{~nm}\left(190 \mathrm{~mW} \cdot \mathrm{cm}^{-2}\right)$, concentration $[\alpha-2-C O O E t]=1,82 \cdot 10^{-4} \mathrm{~mol} \cdot \mathrm{dm}^{-3}$.

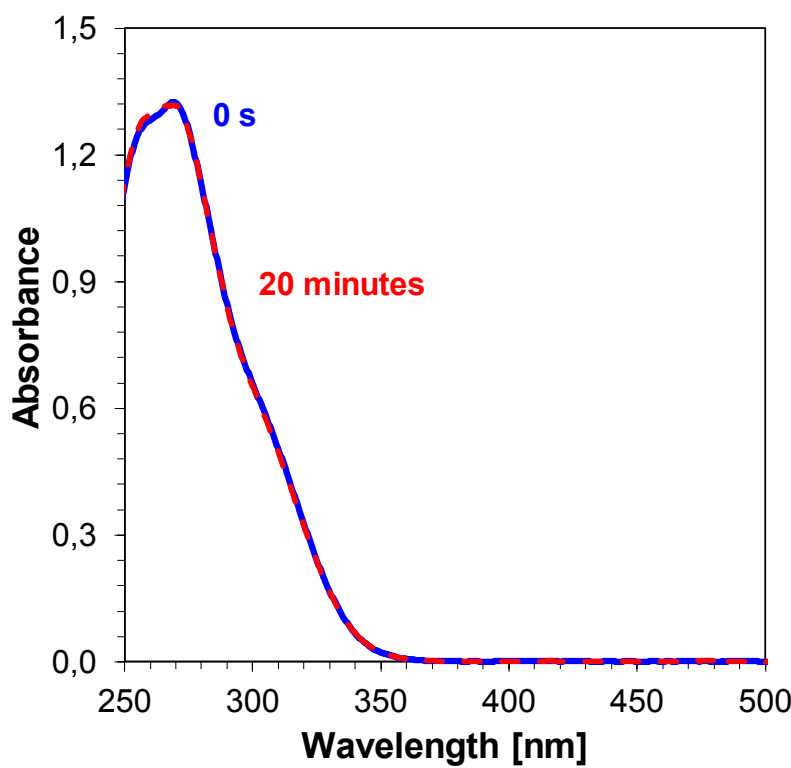

Figure S50. Photolysis of $\beta$-OMe in acetonitrile upon exposure to LED at $365 \mathrm{~nm}\left(190 \mathrm{~mW} \cdot \mathrm{cm}^{-2}\right)$, concentration $[\beta-O M e]=1,11 \cdot 10^{-4} \mathrm{~mol} \cdot \mathrm{dm}^{-3}$. 


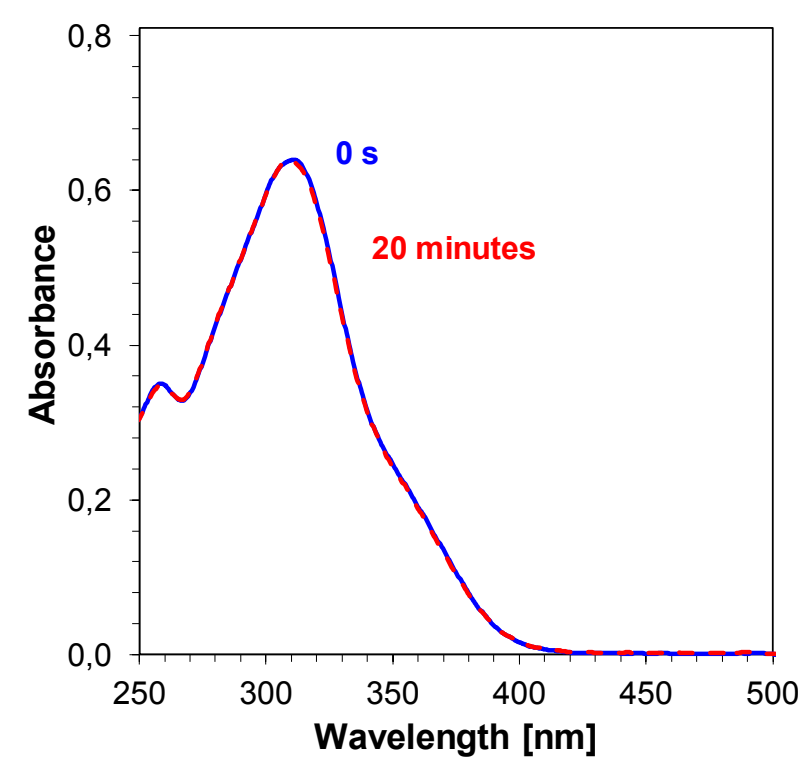

Figure S51. Photolysis of $\beta-P h$ in acetonitrile upon exposure to LED at $365 \mathrm{~nm}\left(190 \mathrm{~mW} \cdot \mathrm{cm}^{-2}\right)$, concentration $[\beta-P h]=5,11 \cdot 10^{-5} \mathrm{~mol} \cdot \mathrm{dm}^{-3}$.

Aryl modified derivatives:

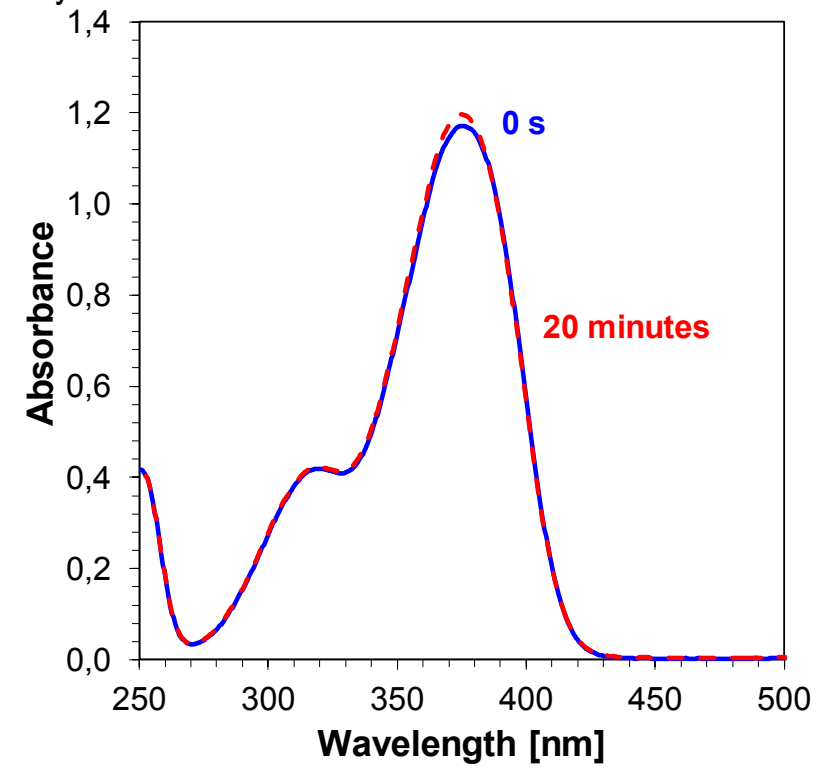

Figure S52. Photolysis of Ar-2,4-OMe in acetonitrile upon exposure to LED at $365 \mathrm{~nm}\left(190 \mathrm{~mW} \cdot \mathrm{cm}^{-2}\right)$, concentration $[$ Ar-2,4-OMe $]=5,09 \cdot 10^{-5} \mathrm{~mol} \cdot \mathrm{dm}^{-3}$.

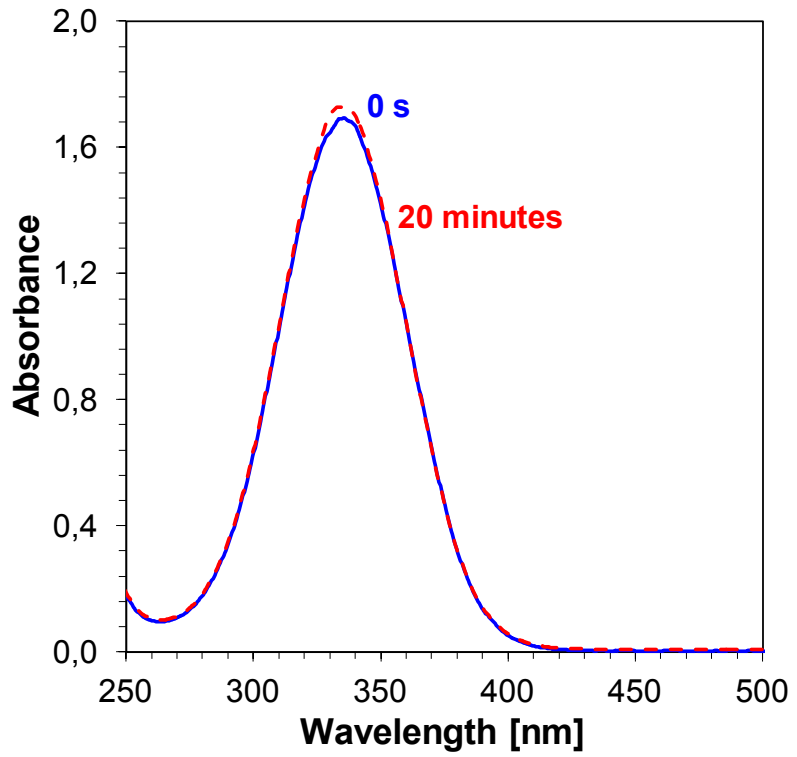

Figure S53. Photolysis of Ar-2,6-OMe in acetonitrile upon exposure to LED at $365 \mathrm{~nm}\left(190 \mathrm{~mW} \cdot \mathrm{cm}^{-2}\right)$, concentration $[$ Ar-2,6-OMe $]=9,95 \cdot 10^{-5} \mathrm{~mol} \cdot \mathrm{dm}^{-3}$. 


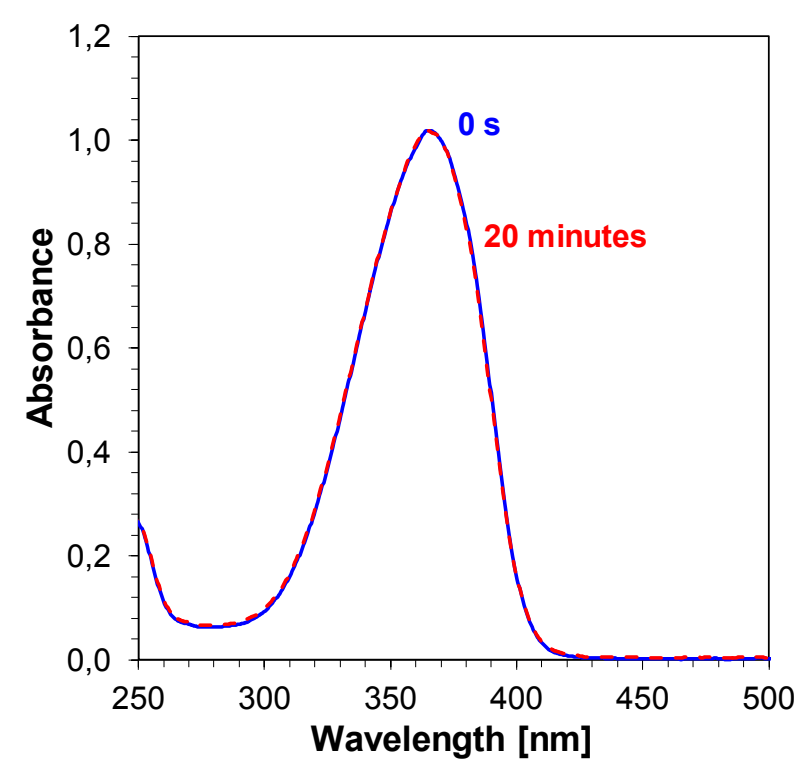

Figure S54. Photolysis of Ar-2,4-6-OMe in acetonitrile upon exposure to LED at $365 \mathrm{~nm}\left(190 \mathrm{~mW} \cdot \mathrm{cm}^{-2}\right)$, concentration $[$ Ar-2,4-6-OMe $]=4,75 \cdot 10^{-5} \mathrm{~mol} \cdot \mathrm{dm}^{-3}$.

Combined derivatives:

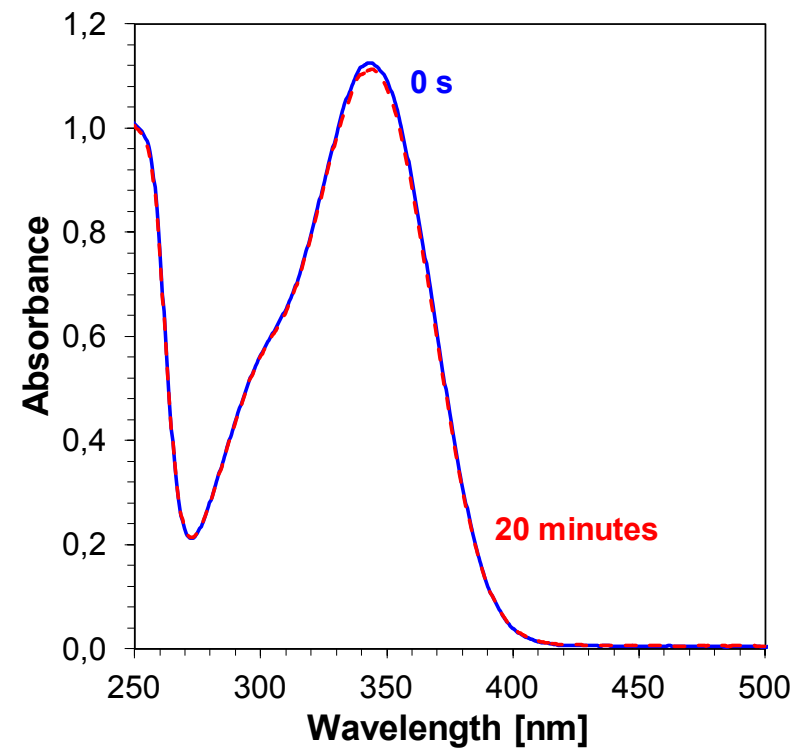

Figure S55. Photolysis of Comb-Me in acetonitrile upon exposure to LED at $365 \mathrm{~nm}\left(190 \mathrm{~mW} \cdot \mathrm{cm}^{-2}\right)$, concentration [Comb-Me] $=1,06 \cdot 10^{-4} \mathrm{~mol} \cdot \mathrm{dm}^{-3}$.

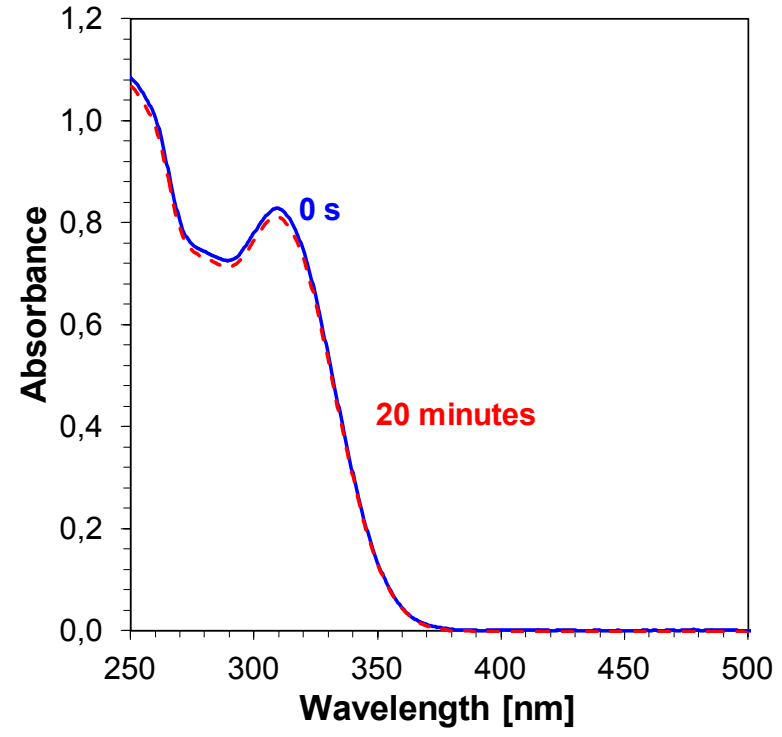

Figure S56. Photolysis of Comb-OMe in acetonitrile upon exposure to LED at $365 \mathrm{~nm}\left(190 \mathrm{~mW} \cdot \mathrm{cm}^{-2}\right)$, concentration [Comb-OMe $]=7,95 \cdot 10^{-5} \mathrm{~mol} \cdot \mathrm{dm}^{-3}$. 


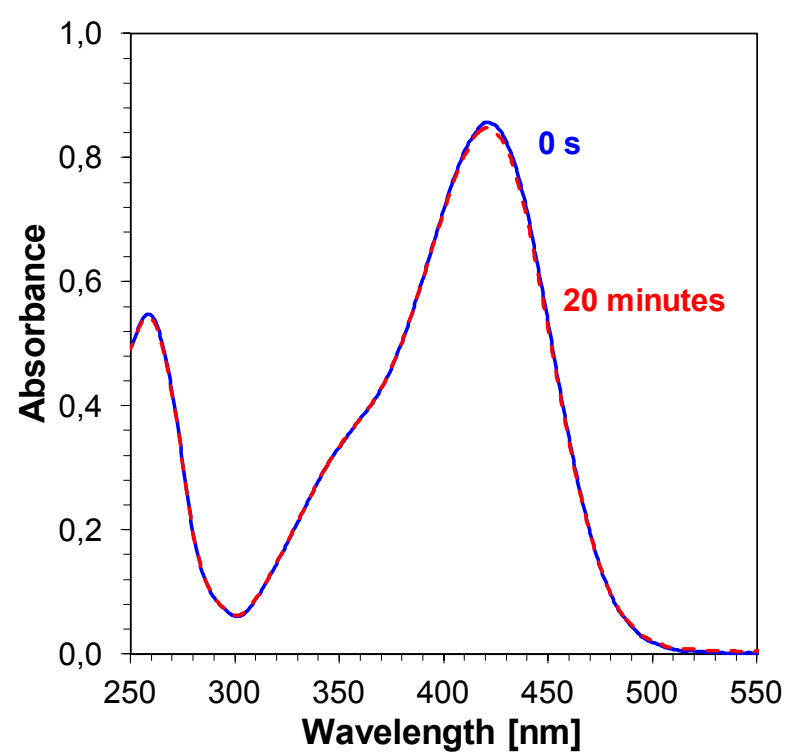

Figure S57. Photolysis of Comb-CN in acetonitrile upon exposure to LED at $365 \mathrm{~nm}\left(190 \mathrm{~mW} \cdot \mathrm{cm}^{-2}\right)$, concentration $[$ Comb-CN $]=8,11 \cdot 10^{-5} \mathrm{~mol} \cdot \mathrm{dm}^{-3}$.

Chromophores that coupled unsuccessfully

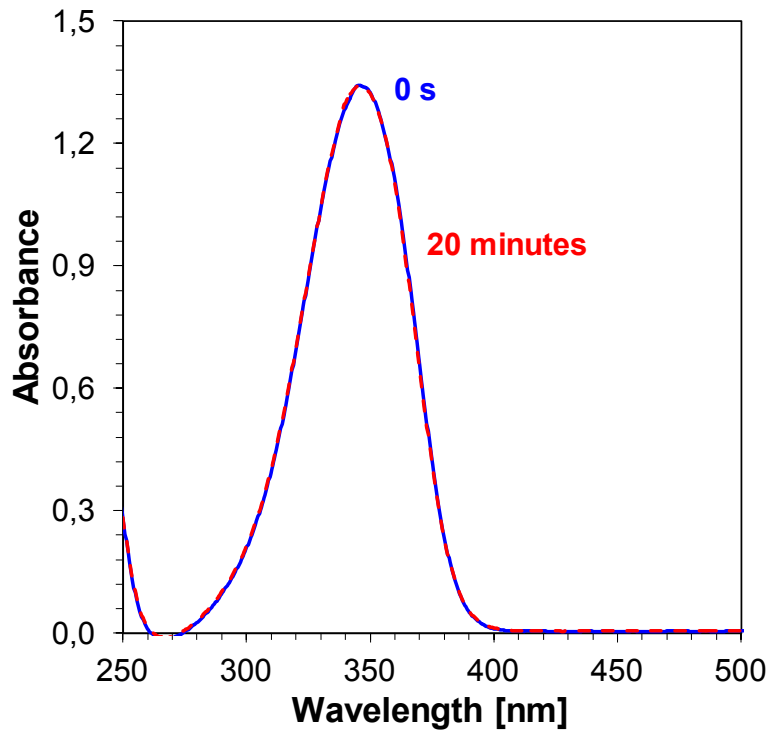

Figure S59. Photolysis of Ar-4-OMe in acetonitrile upon exposure to LED at $365 \mathrm{~nm}\left(190 \mathrm{~mW} \cdot \mathrm{cm}^{-2}\right)$, concentration $[\mathrm{Ar}-4-\mathrm{OMe}]=4,65 \cdot 10^{-5} \mathrm{~mol} \cdot \mathrm{dm}^{-3}$.

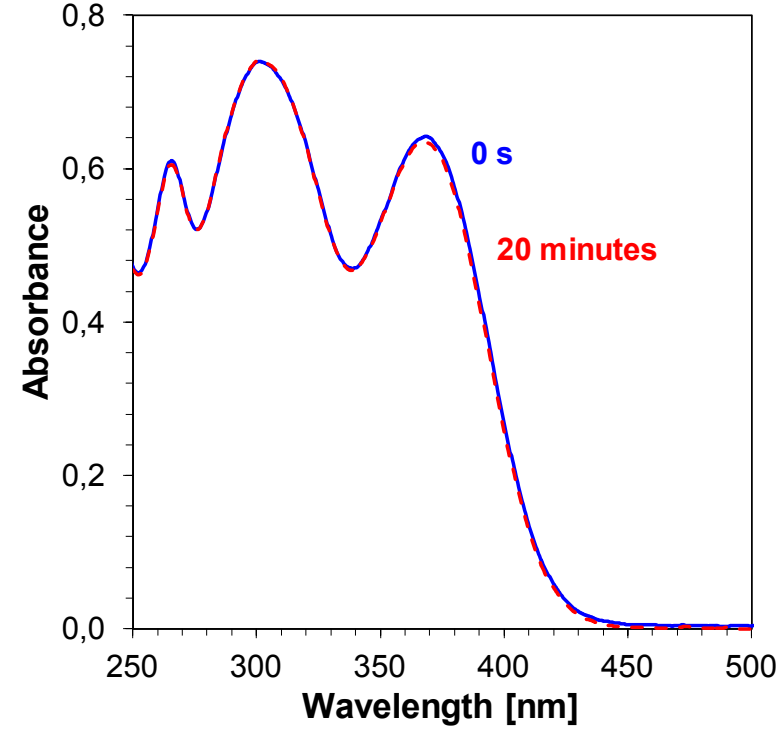

Figure S58. Photolysis of Comb-Ph in acetonitrile upon exposure to LED at $365 \mathrm{~nm}\left(190 \mathrm{~mW} \cdot \mathrm{cm}^{-2}\right)$, concentration [Comb-Ph] $=9,24 \cdot 10^{-5} \mathrm{~mol}^{-\mathrm{dm}^{-3}}$.

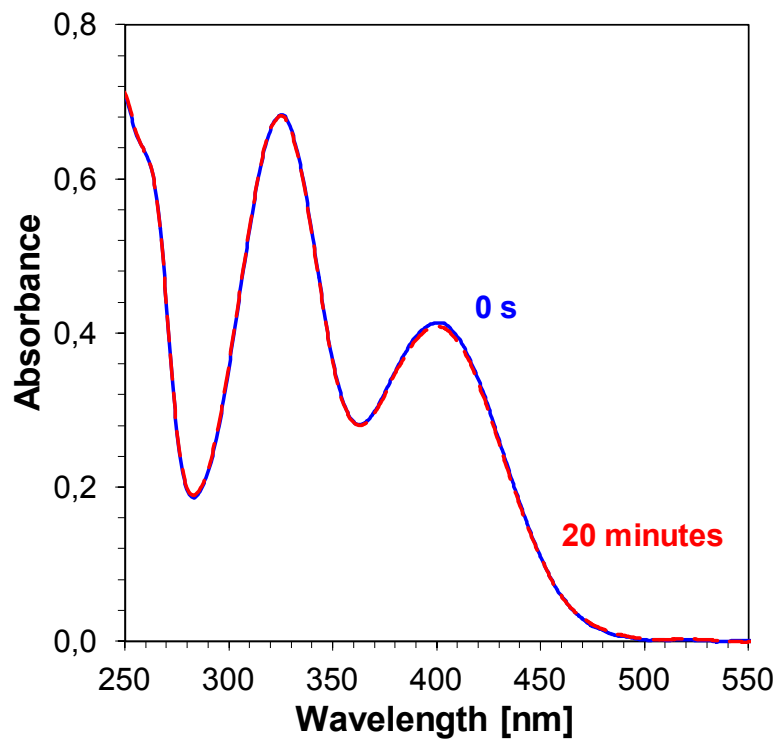

Figure $\mathbf{S 6 0}$. Photolysis of $\beta-\mathbf{C N}$ in acetonitrile upon exposure to $L E D$ at $365 \mathrm{~nm}\left(190 \mathrm{~mW} \cdot \mathrm{cm}^{-2}\right)$, concentration $[\beta-C N]=$ $1,00 \cdot 10^{-4} \mathrm{~mol} \cdot \mathrm{dm}^{-3}$. 
4. Steady state photolysis experiments for iodonium salts

Alpha modified derivatives:

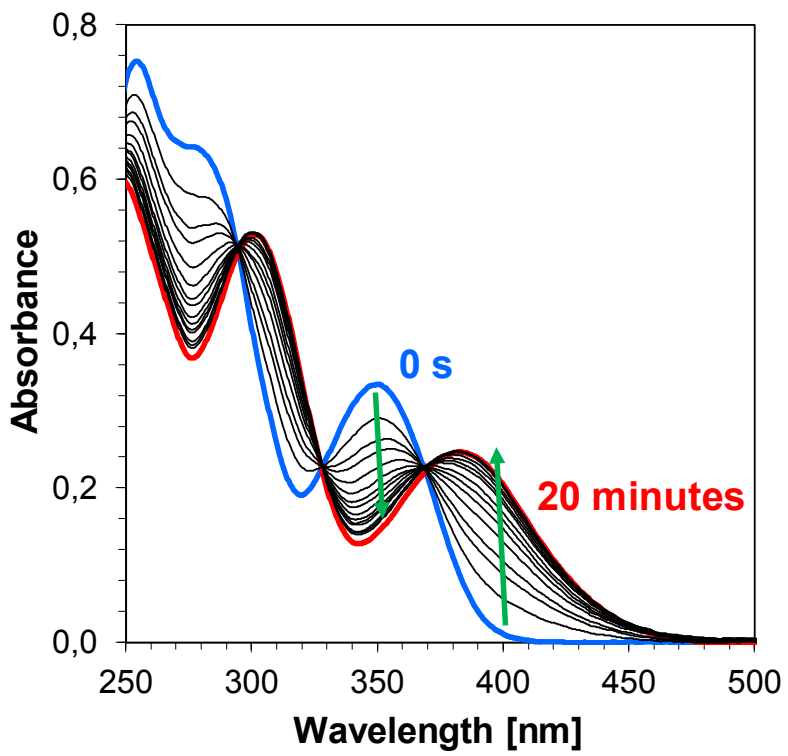

Figure S61. Photolysis of $\alpha-C N-P I$ in acetonitrile upon exposure to LED at $365 \mathrm{~nm}\left(190 \mathrm{~mW} \cdot \mathrm{cm}^{-2}\right)$, concentration $[\mathrm{a}-\mathrm{CN}-\mathrm{Pl}]=4,16 \cdot 10^{-5} \mathrm{~mol} \cdot \mathrm{dm}^{-3}$.

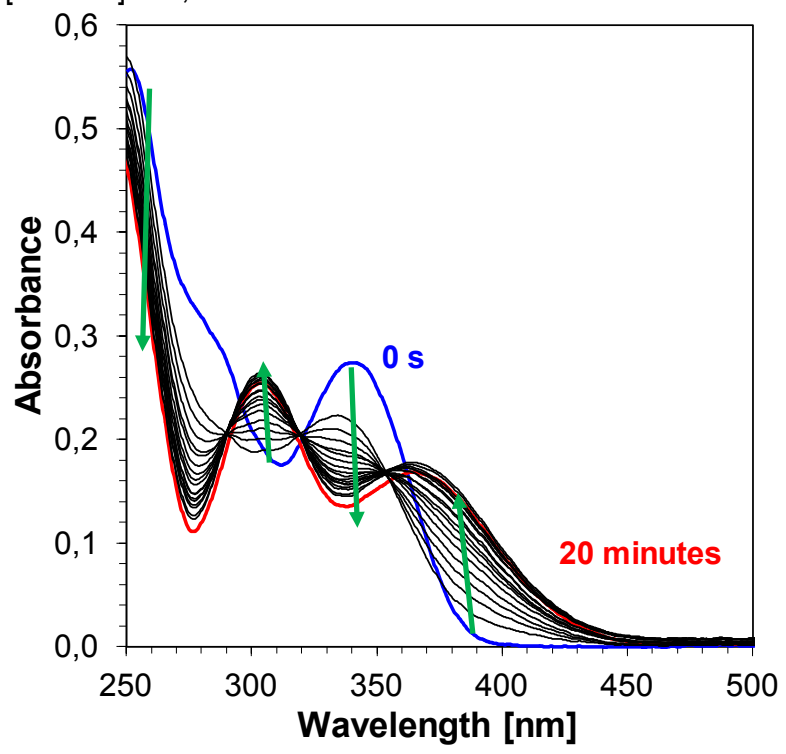

Figure S63. Photolysis of a-COOMe-PI in acetonitrile upon exposure to LED at $365 \mathrm{~nm}\left(190 \mathrm{~mW} \cdot \mathrm{cm}^{-2}\right)$, concentration $[\mathrm{a}-\mathrm{COOMe}-\mathrm{PI}]=3,40 \cdot 10^{-5} \mathrm{~mol} \cdot \mathrm{dm}^{-3}$.

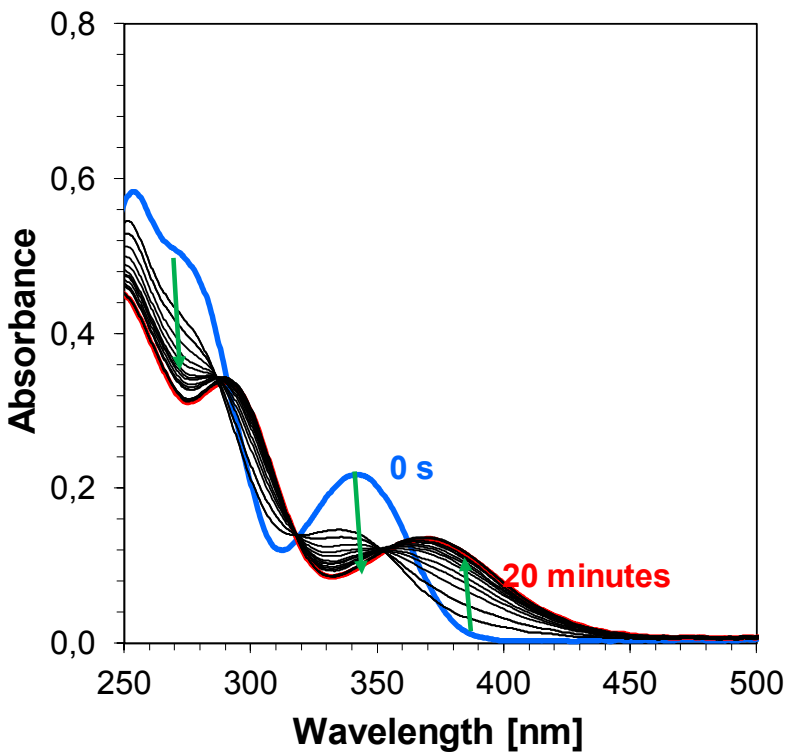

Figure S62. Photolysis of $\mathrm{a}-\mathrm{SO}_{2} \mathrm{Me}-\mathrm{PI}$ in acetonitrile upon exposure to LED at $365 \mathrm{~nm}\left(190 \mathrm{~mW} \cdot \mathrm{cm}^{-2}\right)$, concentration $\left[\alpha-\mathrm{SO}_{2} \mathrm{Me}-\mathrm{PI}\right]=3,35 \cdot 10^{-5} \mathrm{~mol} \cdot \mathrm{dm}^{-3}$.

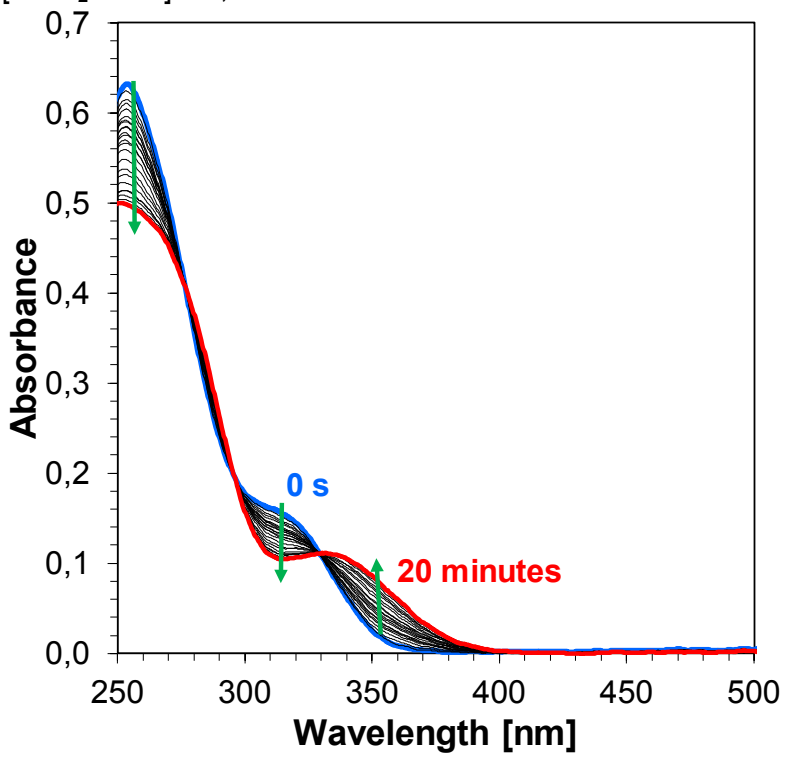

Figure S64. Photolysis of $\alpha$-2-COOEt in acetonitrile upon exposure to LED at $365 \mathrm{~nm}\left(190 \mathrm{~mW} \cdot \mathrm{cm}^{-2}\right)$, concentration $\left[\mathrm{a}-2\right.$-COOEt-PI] $=3,35 \cdot 10^{-5} \mathrm{~mol} \cdot \mathrm{dm}^{-3}$. 
Beta modified derivatives :

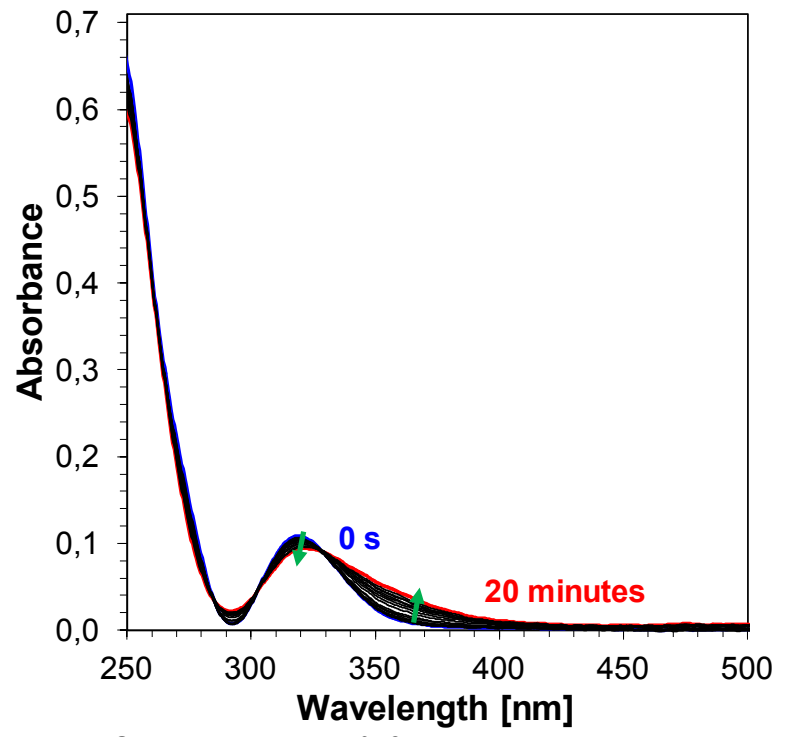

Figure S65. Photolysis of $\beta$-Me-PI in acetonitrile upon exposure to LED at $365 \mathrm{~nm}\left(190 \mathrm{~mW} \cdot \mathrm{cm}^{-2}\right)$, concentration $[\beta-M e-P I]=3,66 \cdot 10^{-5} \mathrm{~mol}^{-} \cdot \mathrm{dm}^{-3}$.

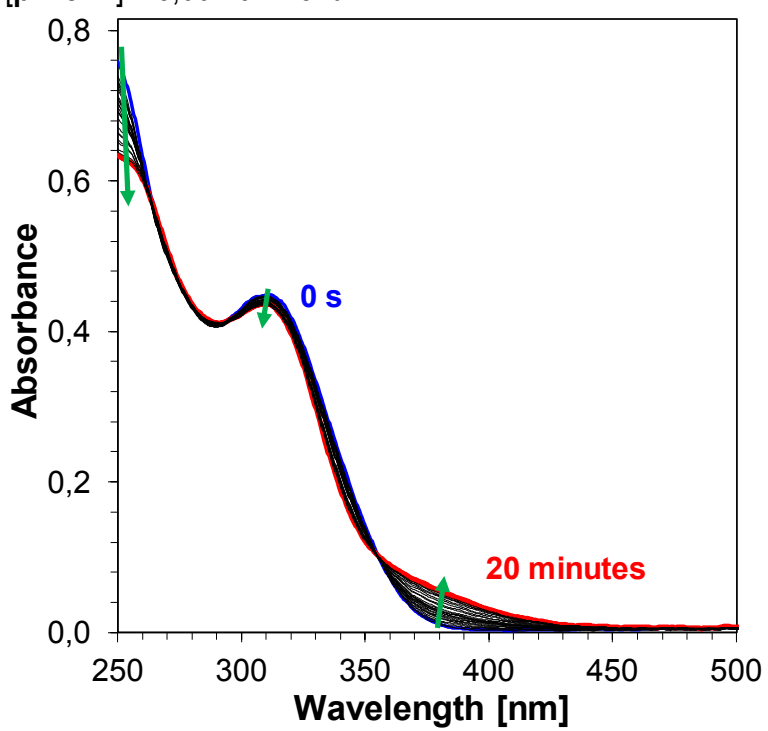

Figure S67. Photolysis of $\beta$-Ph-PI in acetonitrile upon exposure to LED at $365 \mathrm{~nm}\left(190 \mathrm{~mW} \cdot \mathrm{cm}^{-2}\right)$, concentration $[\beta-P h-P I]=3,68 \cdot 10^{-5} \mathrm{~mol}^{-\mathrm{dm}^{-3}}$.

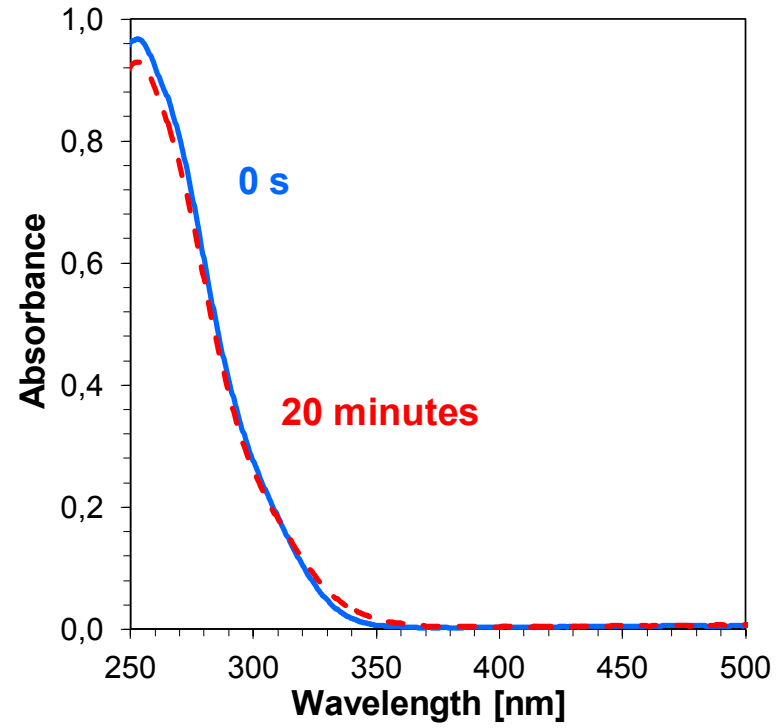

Figure S66. Photolysis of $\beta$-OMe-PI in acetonitrile upon exposure to LED at $365 \mathrm{~nm}\left(190 \mathrm{~mW} \cdot \mathrm{cm}^{-2}\right)$, concentration $[\beta-\mathrm{OMe}-\mathrm{PI}]=4,80 \cdot 10^{-5} \mathrm{~mol} \cdot \mathrm{dm}^{-3}$. 
Aryl modified derivatives:

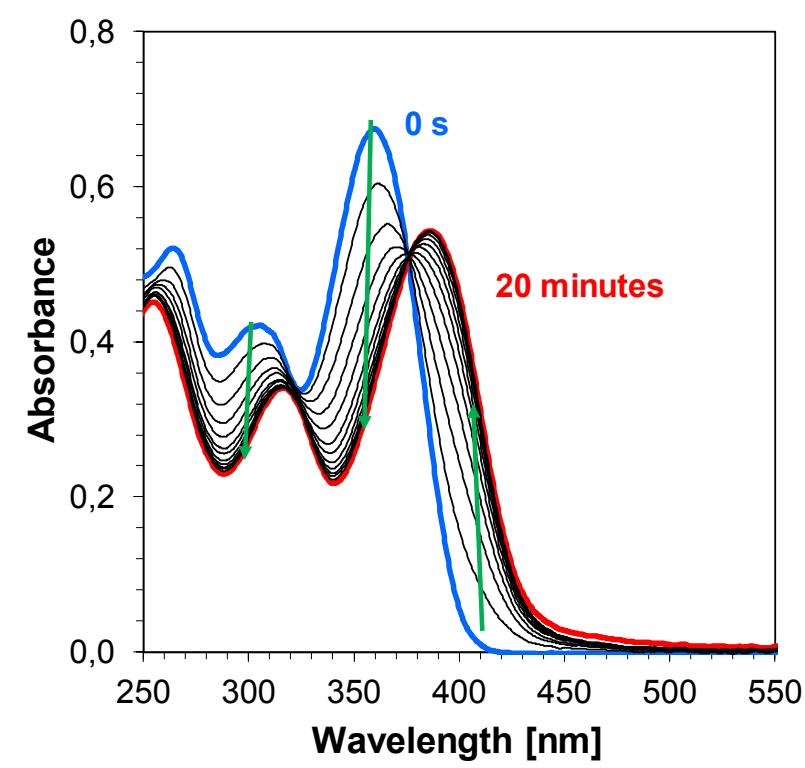

Figure S68. Photolysis of Ar-2,4-OMe-PI in acetonitrile upon exposure to LED at $365 \mathrm{~nm}\left(190 \mathrm{~mW} \cdot \mathrm{cm}^{-2}\right)$, concentration $[\mathrm{Ar}-2,4-\mathrm{OMe}-\mathrm{PI}]=3,97 \cdot 10^{-5} \mathrm{~mol} \cdot \mathrm{dm}^{-3}$.

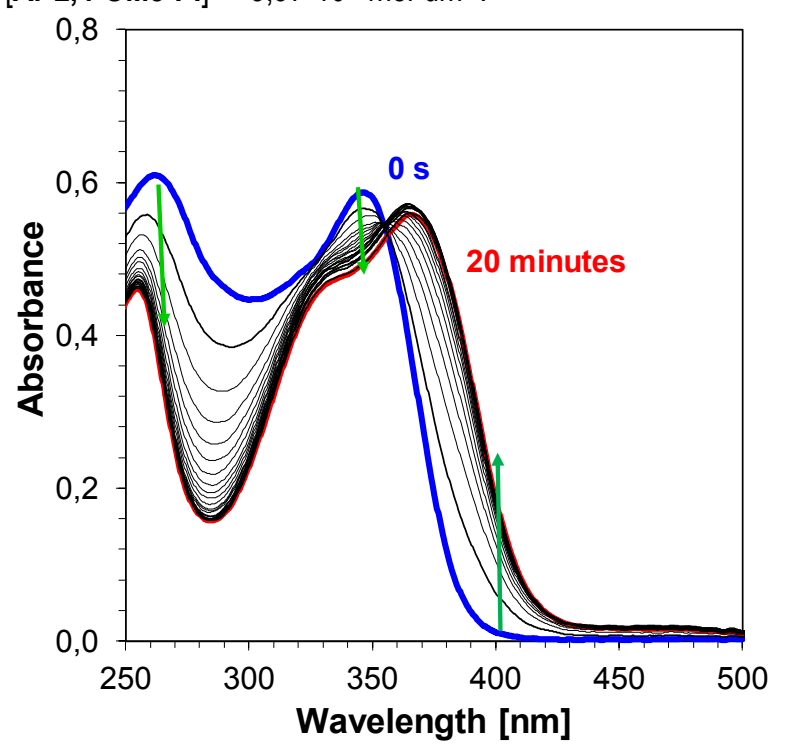

Figure S70. Photolysis of Ar-2,4-6-OMe-PI in acetonitrile upon exposure to LED at $365 \mathrm{~nm}\left(190 \mathrm{~mW} \cdot \mathrm{cm}^{-2}\right)$, concentration $[\mathrm{Ar}-2,4-6-\mathrm{OMe}-\mathrm{PI}]=4,16 \cdot 10^{-5} \mathrm{~mol} \cdot \mathrm{dm}^{-3}$.

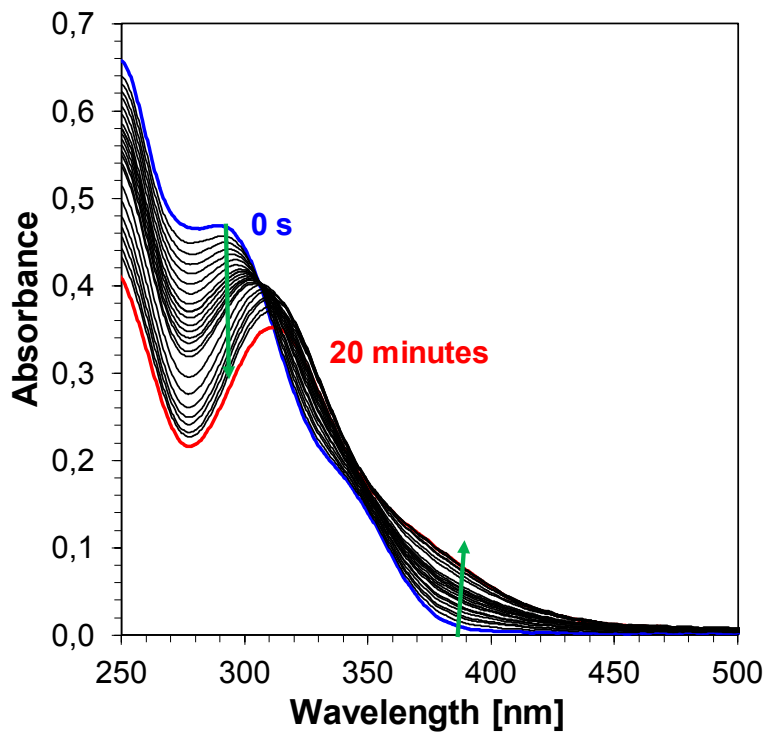

Figure S69. Photolysis of Ar-2,6-OMe-PI in acetonitrile upon exposure to LED at $365 \mathrm{~nm}\left(190 \mathrm{~mW} \cdot \mathrm{cm}^{-2}\right)$, concentration $[\mathrm{Ar}-2,6-\mathrm{OMe}-\mathrm{PI}]=3,81 \cdot 10^{-5} \mathrm{~mol} \cdot \mathrm{dm}^{-3}$. 
Combined derivatives:

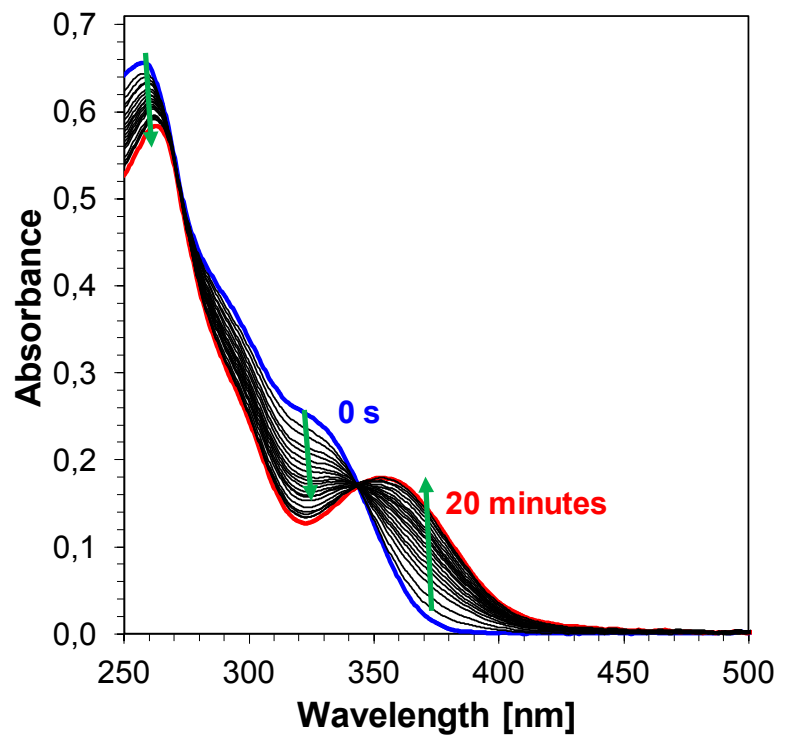

Figure S71. Photolysis of Comb-Me-PI in acetonitrile upon exposure to LED at $365 \mathrm{~nm}\left(190 \mathrm{~mW} \cdot \mathrm{cm}^{-2}\right)$, concentration [Comb-Me-PI] $=3,19 \cdot 10^{-5} \mathrm{~mol} \cdot \mathrm{dm}^{-3}$.

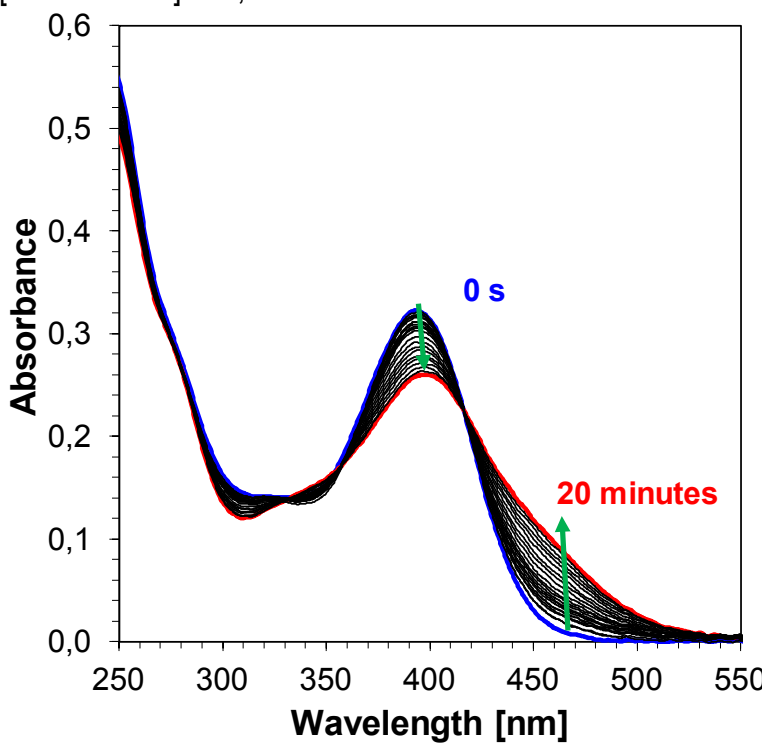

Figure S73. Photolysis of Comb-CN-PI in acetonitrile upon exposure to LED at $365 \mathrm{~nm}\left(190 \mathrm{~mW} \cdot \mathrm{cm}^{-2}\right)$, concentration [Comb-CN-PI] $=3,27 \cdot 10^{-5} \mathrm{~mol} \cdot \mathrm{dm}^{-3}$.

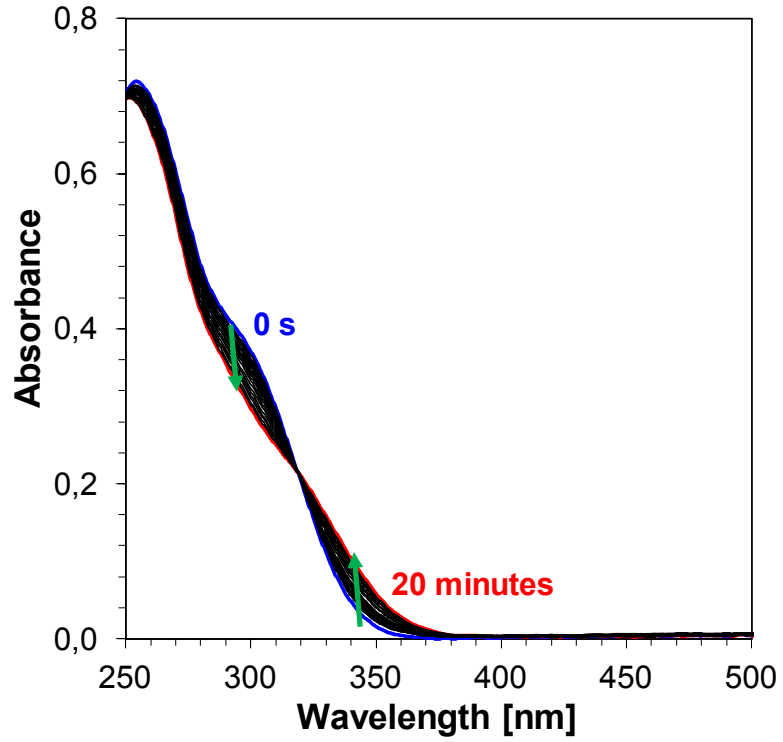

Figure S72. Photolysis of Comb-OMe-PI in acetonitrile upon exposure to LED at $365 \mathrm{~nm}\left(190 \mathrm{~mW} \cdot \mathrm{cm}^{-2}\right)$, concentration [Comb-OMe-PI] $=3,75 \cdot 10^{-5} \mathrm{~mol} \cdot \mathrm{dm}^{-3}$.

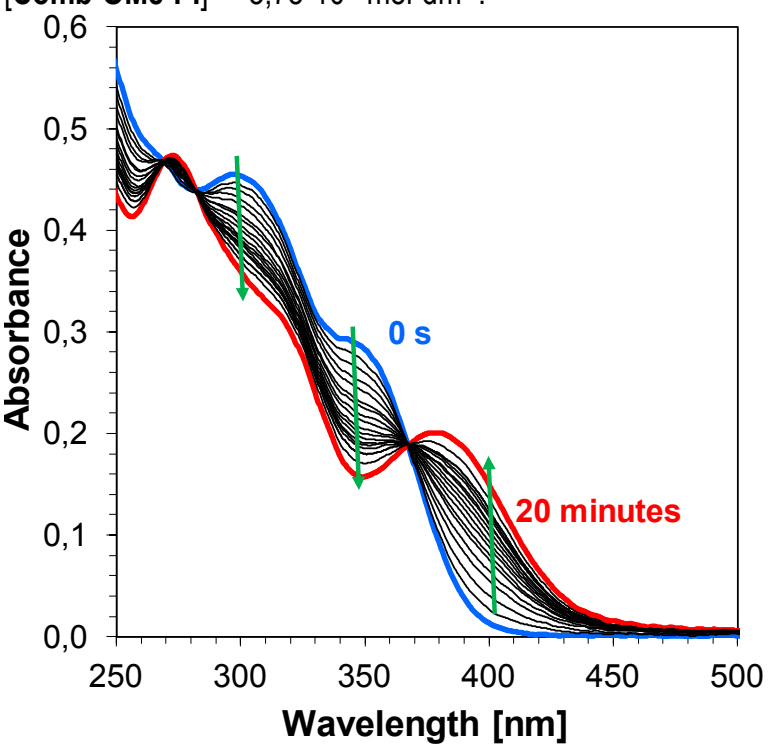

Figure S74. Photolysis of Comb-Ph-PI in acetonitrile upon exposure to LED at $365 \mathrm{~nm}\left(190 \mathrm{~mW} \cdot \mathrm{cm}^{-2}\right)$, concentration [Comb-Ph-PI] $=3,16 \cdot 10^{-5} \mathrm{~mol} \cdot \mathrm{dm}^{-3}$. 
Steady state photolysis experiments for selected iodonium salts under different LEDs:

- For $\alpha-C N-P I:$

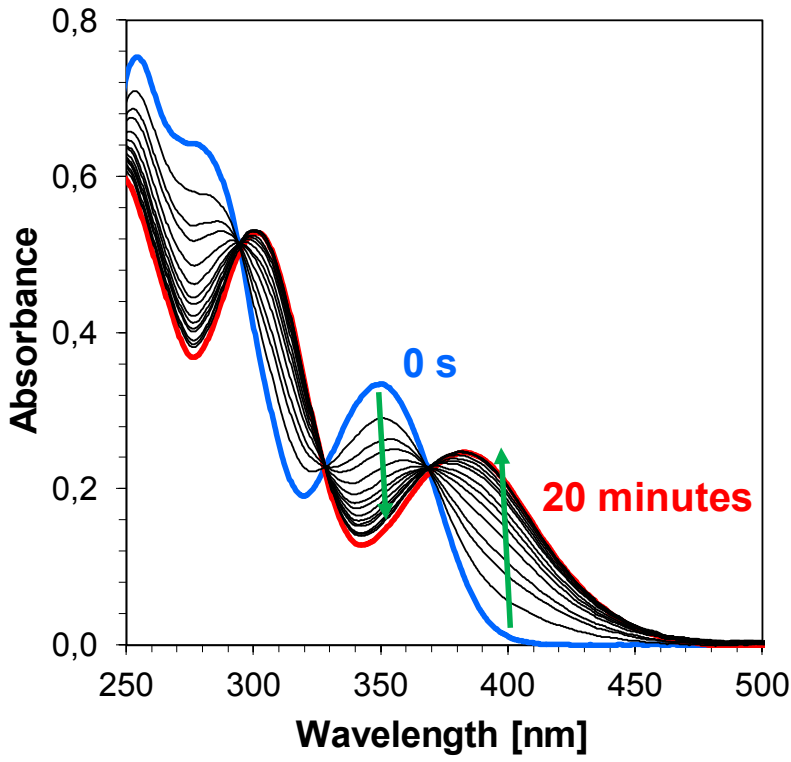

Figure S75. Photolysis of $\alpha-\mathrm{CN}-\mathrm{PI}$ in acetonitrile upon exposure to LED at $365 \mathrm{~nm}\left(190 \mathrm{~mW} \cdot \mathrm{cm}^{-2}\right)$, concentration [a$\mathrm{CN}-\mathrm{PI}]=4,16 \cdot 10^{-5} \mathrm{~mol} \cdot \mathrm{dm}^{-3}$.

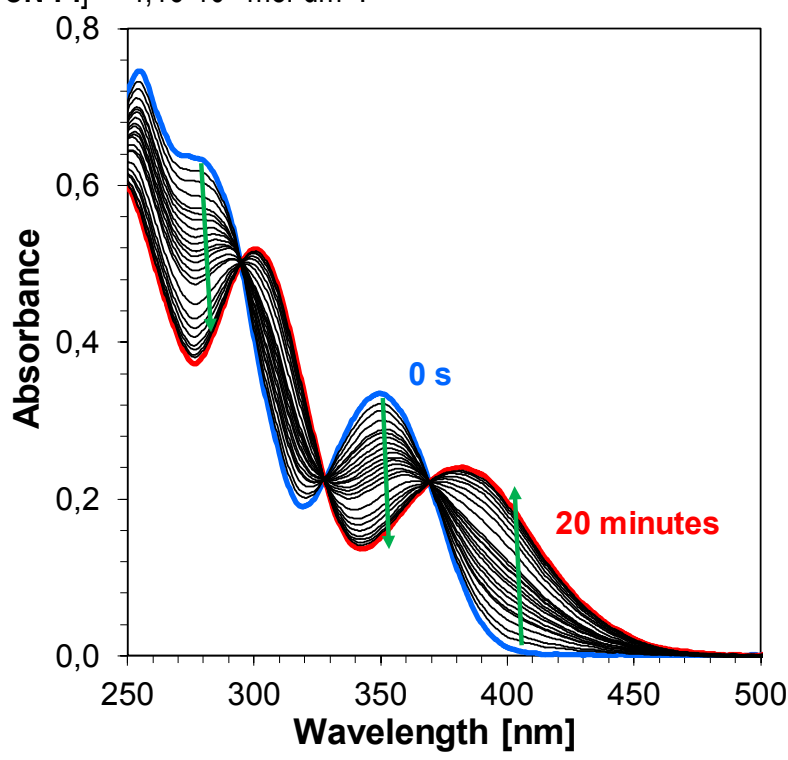

Figure S77. Photolysis of $\alpha-C N-P I$ in acetonitrile upon exposure to LED at $385 \mathrm{~nm}\left(190 \mathrm{~mW} \cdot \mathrm{cm}^{-2}\right)$, concentration [a$\mathrm{CN}-\mathrm{PI}]=4,16 \cdot 10^{-5} \mathrm{~mol} \cdot \mathrm{dm}^{-3}$.

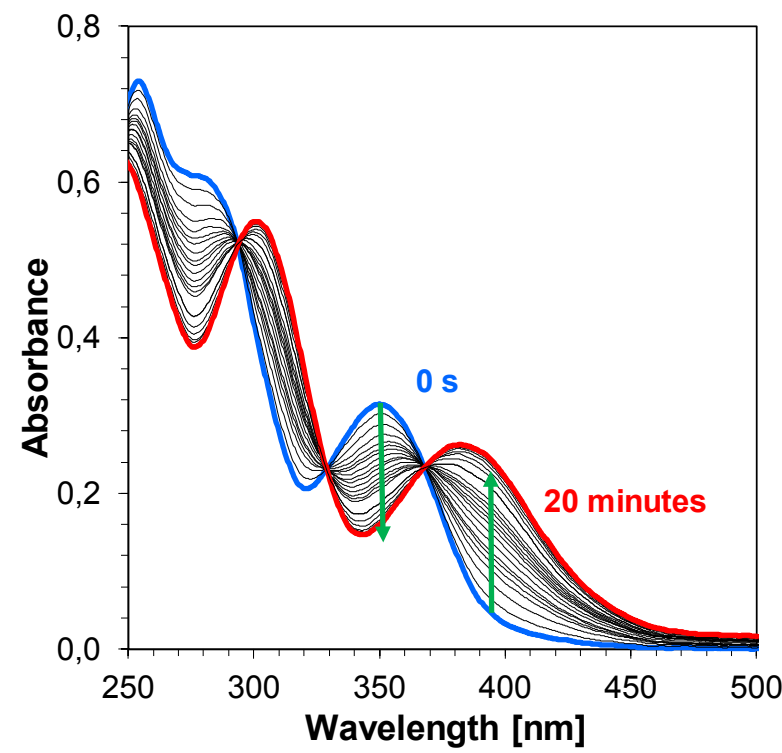

Figure S76. Photolysis of $\alpha-C N-P I$ in acetonitrile upon exposure to LED at $375 \mathrm{~nm}\left(190 \mathrm{~mW} \cdot \mathrm{cm}^{-2}\right)$, concentration $[\alpha-C N-P I]=4,16 \cdot 10^{-5} \mathrm{~mol} \cdot \mathrm{dm}^{-3}$.

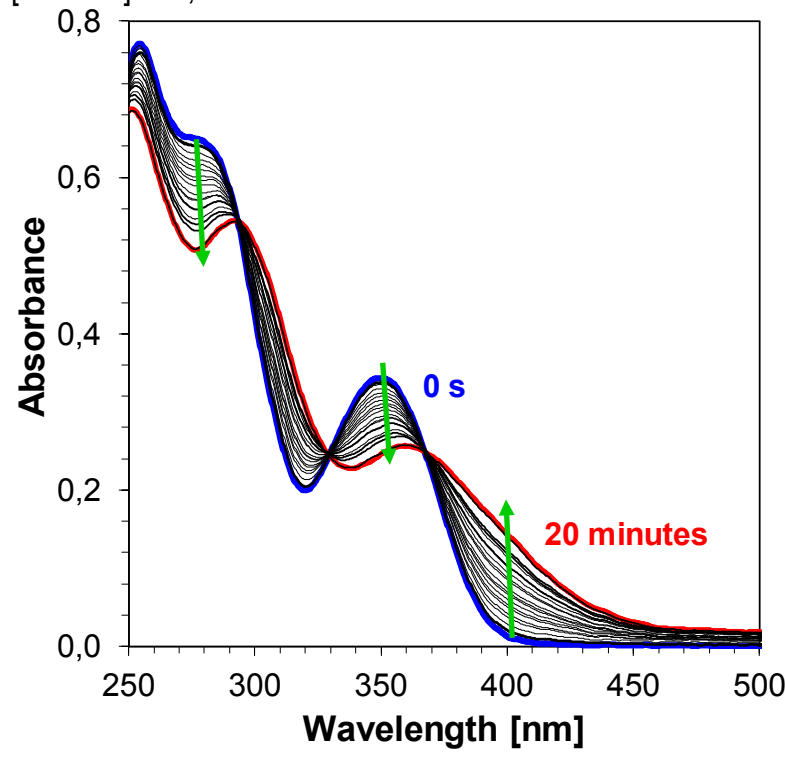

Figure S78. Photolysis of $\alpha-C N-P I$ in acetonitrile upon exposure to LED at $405 \mathrm{~nm}\left(190 \mathrm{~mW} \cdot \mathrm{cm}^{-2}\right)$, concentration $[\alpha-C N-P I]=4,16 \cdot 10^{-5} \mathrm{~mol} \cdot \mathrm{dm}^{-3}$. 
- For Ar-2,4-OMe-PI:

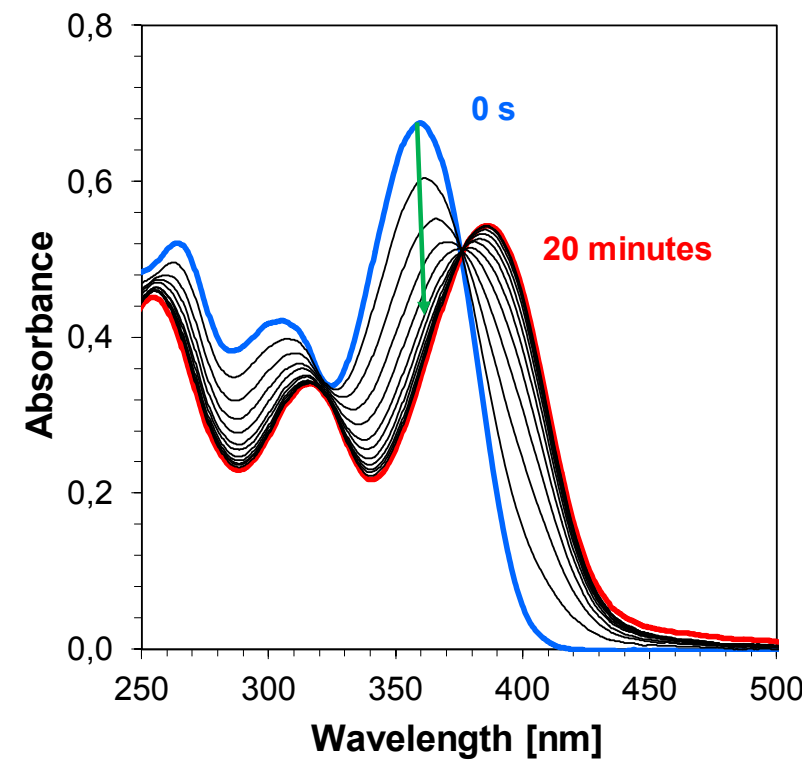

Figure S79. Photolysis of Ar-2,4-OMe-PI in acetonitrile upon exposure to LED at $365 \mathrm{~nm}\left(190 \mathrm{~mW} \cdot \mathrm{cm}^{-2}\right)$, concentration $[\mathrm{Ar}-2,4-\mathrm{OMe}-\mathrm{PI}]=3,97 \cdot 10^{-5} \mathrm{~mol} \cdot \mathrm{dm}^{-3}$.

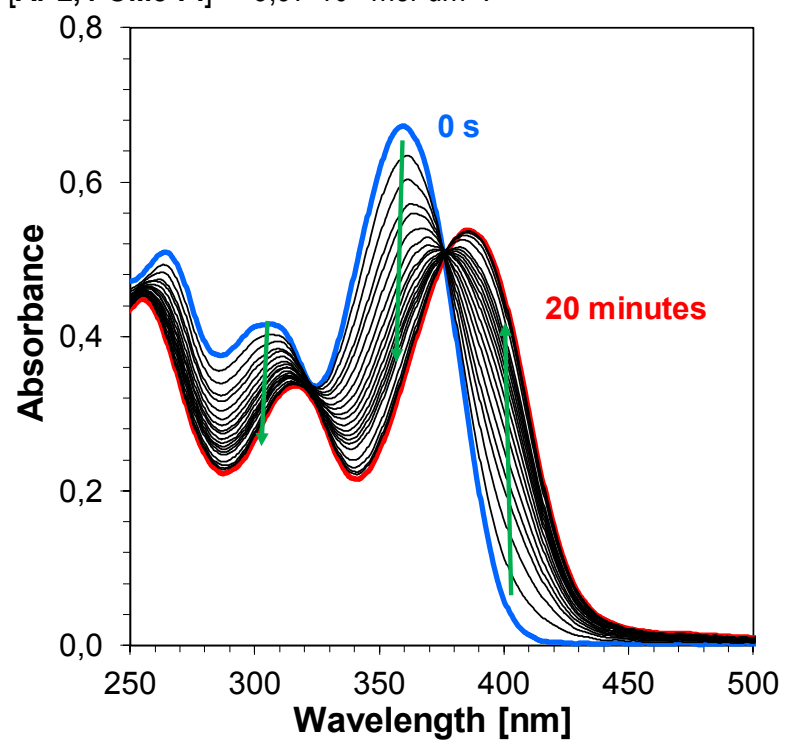

Figure S81. Photolysis of Ar-2,4-OMe-PI in acetonitrile upon exposure to LED at $385 \mathrm{~nm}\left(190 \mathrm{~mW} \cdot \mathrm{cm}^{-2}\right)$, concentration $[\mathrm{Ar}-2,4-\mathrm{OMe}-\mathrm{PI}]=3,97 \cdot 10^{-5} \mathrm{~mol} \cdot \mathrm{dm}^{-3}$.

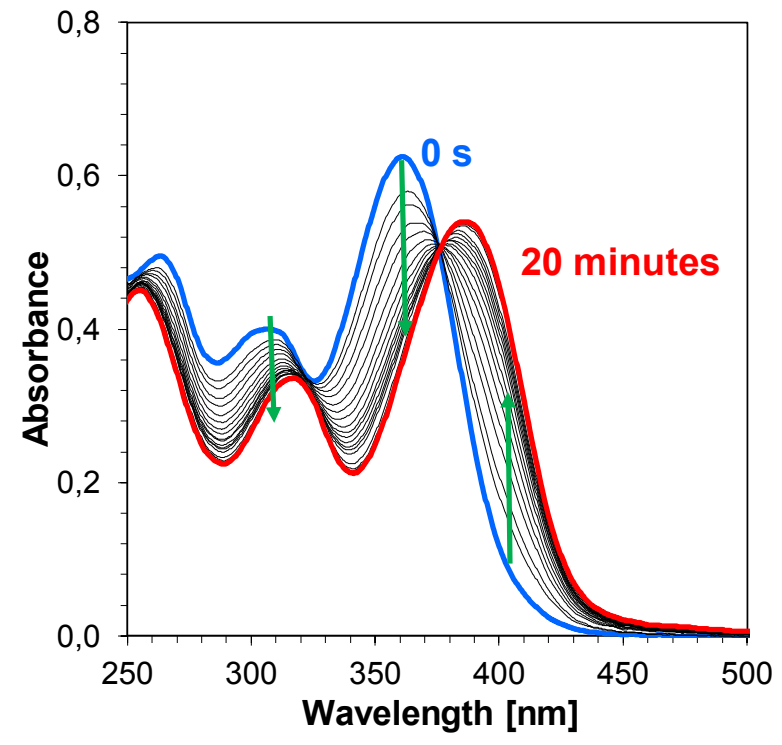

Figure S80. Photolysis of Ar-2,4-OMe-PI in acetonitrile upon exposure to LED at $375 \mathrm{~nm}\left(190 \mathrm{~mW} \cdot \mathrm{cm}^{-2}\right)$, concentration $[\mathrm{Ar}-2,4-\mathrm{OMe}-\mathrm{PI}]=3,97 \cdot 10^{-5} \mathrm{~mol} \cdot \mathrm{dm}^{-3}$.

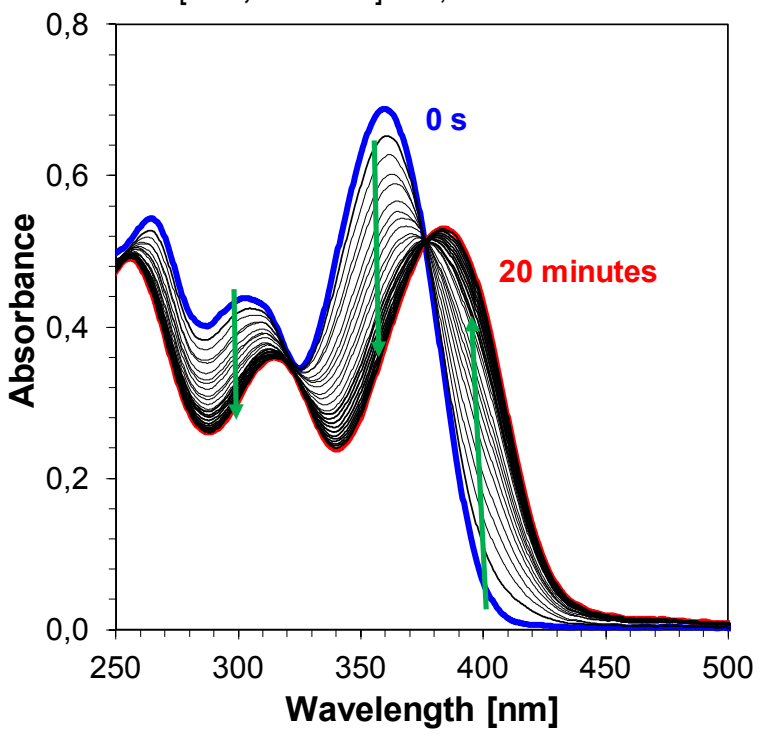

Figure S82. Photolysis of Ar-2,4-OMe-PI in acetonitrile upon exposure to LED at $405 \mathrm{~nm}\left(190 \mathrm{~mW} \cdot \mathrm{cm}^{-2}\right)$, concentration $[\mathrm{Ar}-2,4-\mathrm{OMe}-\mathrm{PI}]=3,97 \cdot 10^{-5} \mathrm{~mol} \cdot \mathrm{dm}^{-3}$. 


\section{Quantum efficiency of acid generation}

Measurements of quantum efficiency of superacid were carried out according to procedure described in literature ${ }^{9}$. As a light source during irradiation M340L4 UV-LED (power 0,7 A, irradiance $\mathrm{I}_{0}$ $=53 \mathrm{~mW} \cdot \mathrm{cm}^{-2}$, from Thorlabs Inc. USA) powered by a DC2200 regulated power supply (from Thorlabs Inc. USA) was used which emitted light with maximum at $340 \mathrm{~nm}$. Measurements were proceed in CVH100-CV holder (from Thorlabs Inc. USA). Absorption spectra were recorded using the SilverNova spectrometer (StellarNet, Inc., USA) in combination with a broadband tungsten-deuterium UV-Vis light source (StellarNet, Inc., USA) in a quartz cuvette with $1,0 \mathrm{~cm}$ optical path.

Irradiated solution were prepared by dissolution of photoinitiators in acetonitrile, the absorbance of solutions was greater than 2,5 in whole emission range of used LED to ensure a nearly $100 \%$ absorption of the incident photons. This conditions allowed to make an assumption:

,where:

$$
n_{a b s}=n_{e m i t}
$$

- $\mathrm{n}_{\mathrm{abs}}$ - amount of absorbed photons by irradiated sample

- $n_{\text {emit }}$ - amount of photons emitted by light source

In these conditions amount of photons absorbed by sample is known what makes it easier to determine quantum efficiency. This value was determined using equation presented below:

$$
\phi_{H A}=\frac{[H A]}{n_{a b s}}=\frac{[H A]}{n_{\text {emit }}}
$$

,where:

- $[\mathrm{HA}]$ - concentration of acid generated during irradiation of sample

- $n_{\text {abs }}$ - amount of photons absorbed by irradiated sample

- $n_{\text {emit }}$ - amount of photons emitted by light source

In order to determine concentration of acid generated during irradiation of sample Rhodamine $B$ base was used as an acid indicator. Rhodamine $B$ base has two forms which differ significantly in the absorption spectrum (fig. Xxx). This compound is very sensitive and react with minimal amount of acid which makes this compound the perfect acid indicator. ${ }^{10}$
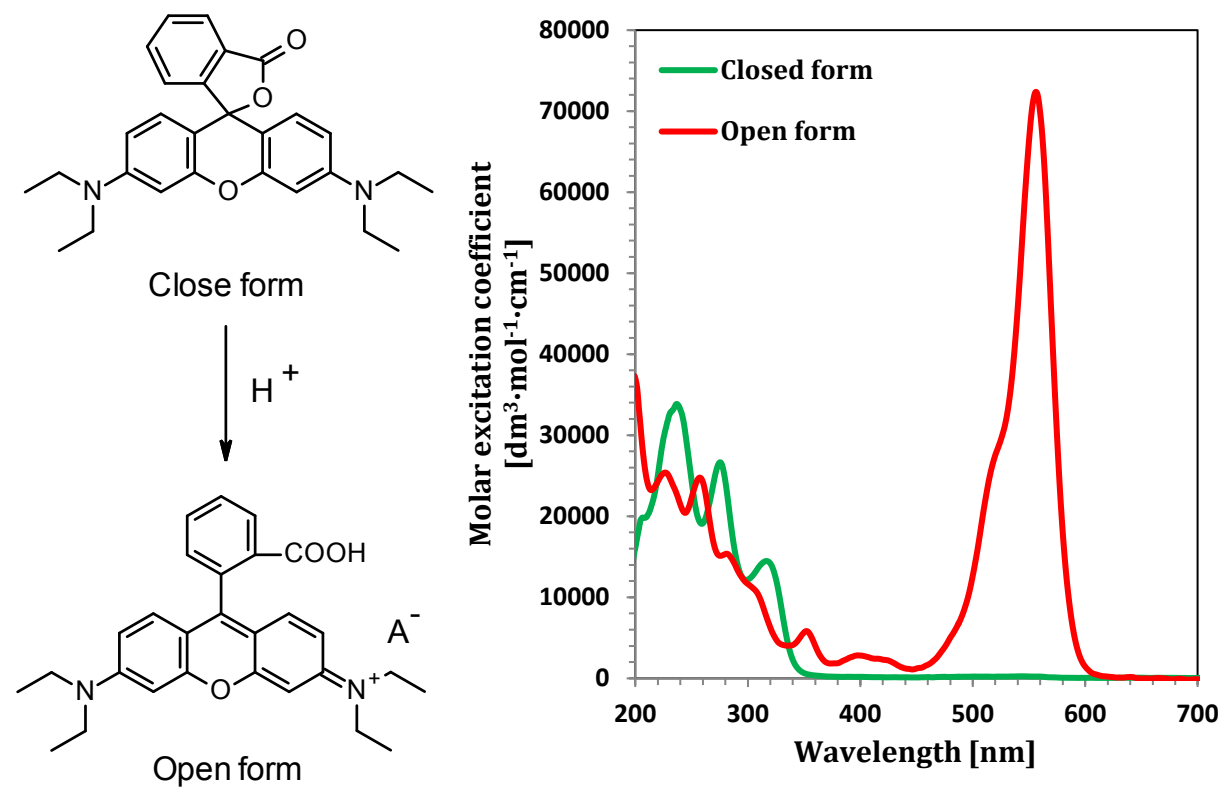

Figure S83. Opening of Rhodamine B base (left) and absorption spectrum of both forms of this compound (right). 
The precise acid concentration value was read from the calibration curve. During calibration curve preparation $p$-toluenosulfonic acid was used as acid standard. Measurements were carried out in acetonitrile and absorbance at $555 \mathrm{~nm}$ was monitored. Behavior of absorbance spectra of Rhodamine B base during acid titration and prepared calibration curve are presented in Figure 83.
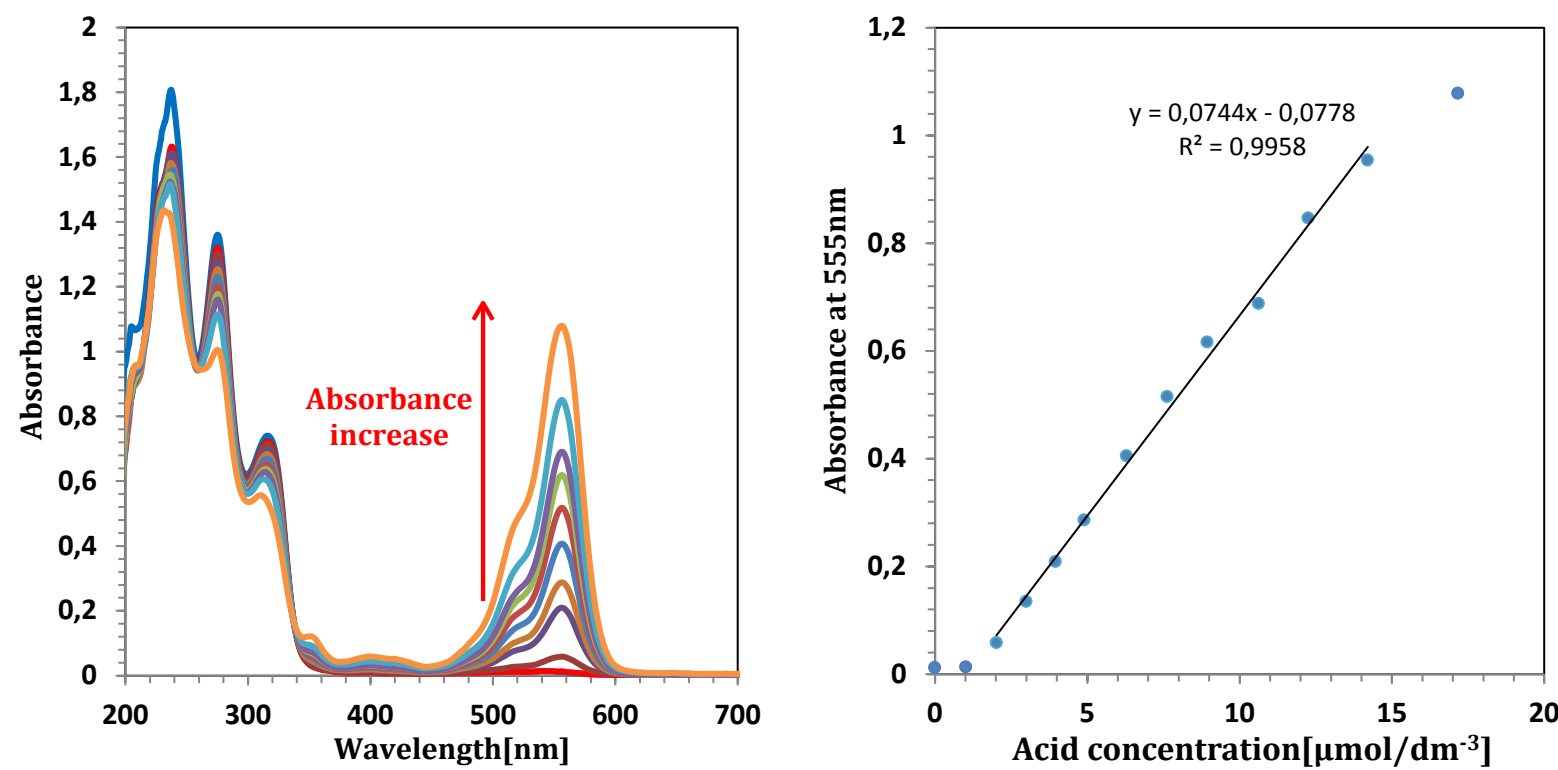

Figure S84. Behavior of absorbance spectra of Rhodamine B base during acid titration (left) and prepared calibration curve (right).

In order to determine amount of photons emitted by used light source actinometric measurements were carried out using Hatchard-Parker actinometer (potassium tris(oxalate)ferrate(III) $)^{11}$. Measurements were proceed according to standard IUPAC procedure. ${ }^{12}$

Samples of iodonium salts in acetonitrile were irradiated at the same power output of light source at different times of irradiation. After irradiation, standard solution of rhodamine B base in acetonitrile was added to irradiated sample and absorbance at $555 \mathrm{~nm}$ was recorded. Reference samples were prepared in the same way as irradiaded sample but rhodamine B base was added to them with no irradiation. Concentration of generated acid was determined from difference between absorbance at $555 \mathrm{~nm}$ of reference and irradiated samples. The irradiation time was selected so as to obtain a satisfactory change in absorbance while not leading to a decomposition of more than $10 \%$ of iodonium salt. 
6. Performance of the benzylidene derivatives as initiators for cationic photopolymerization of vinyl monomer

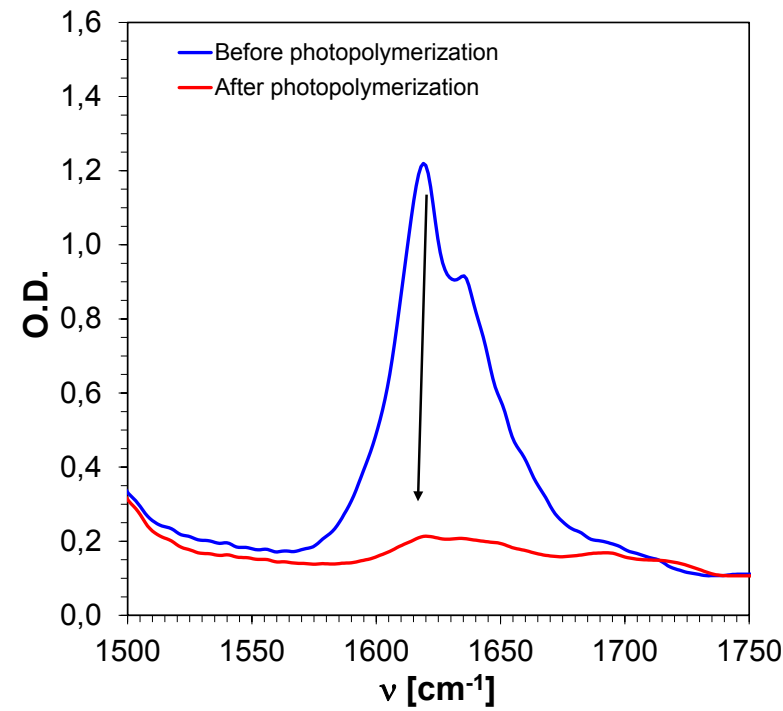

Figure S85. Spectra before and after photopolymerization of vinyl monomer with initiator $\alpha-\mathrm{CN}-\mathrm{PI}$ under LED 365nm.

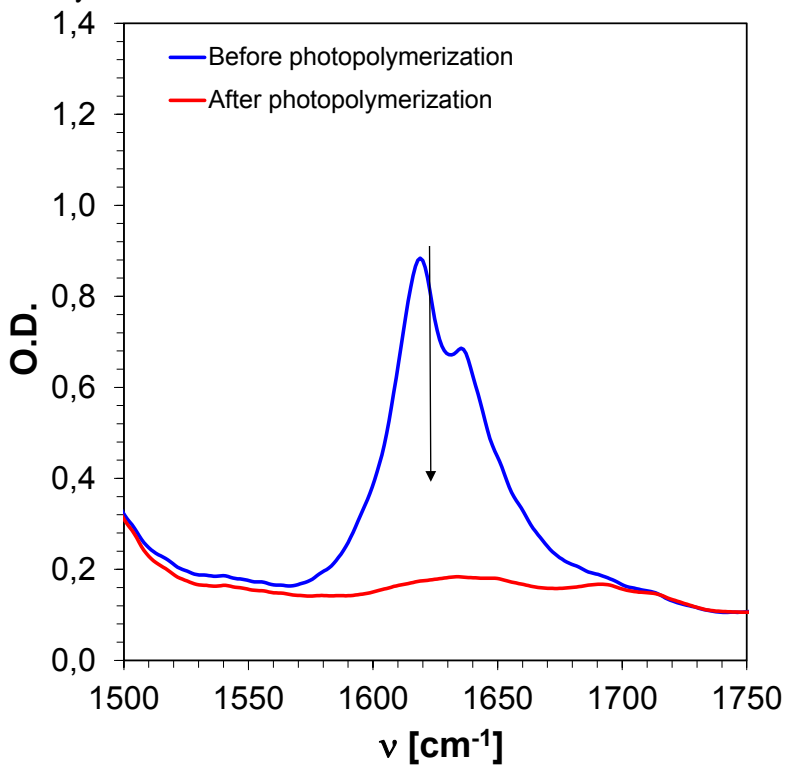

Figure S87. Spectra before and after photopolymerization of vinyl monomer with initiator Ar-2,4-OMe-PI under LED $365 \mathrm{~nm}$.

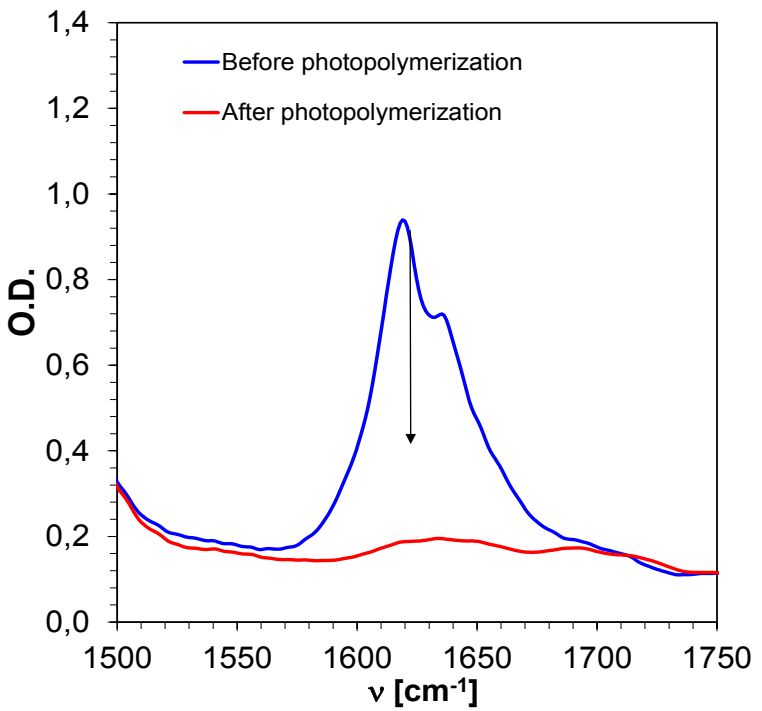

Figure S86.Spectra before and after photopolymerization of vinyl monomer with initiator $\beta$-Ph-PI under LED 365nm.

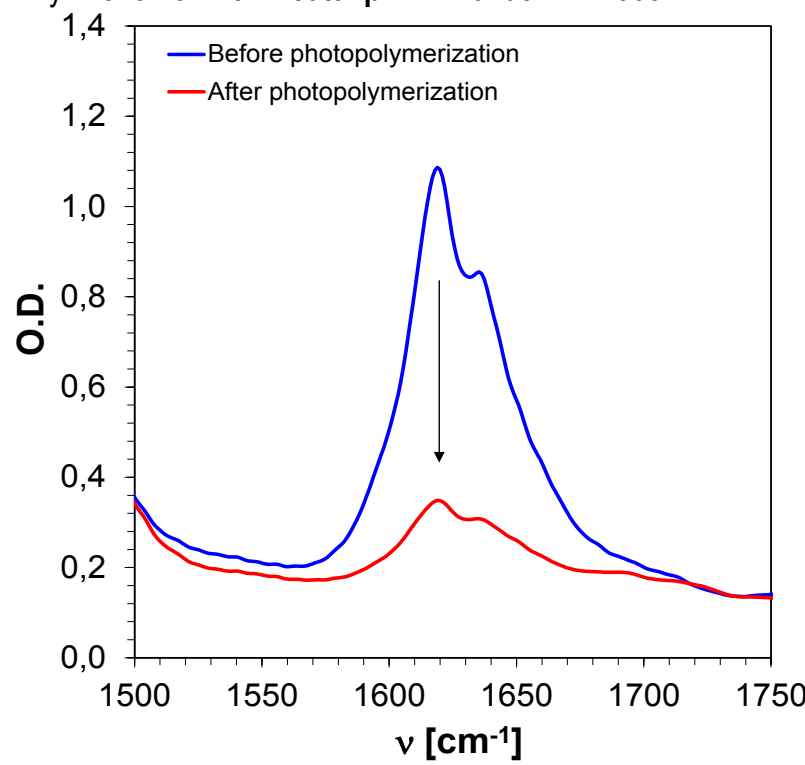

Figure S88. Spectra before and after photopolymerization of vinyl monomer with initiator Comb-Me-PI under LED 365nm. 
7. Performance of the benzylidene derivatives as initiators for cationic photopolymerization of cycloaliphatic epoxy resin

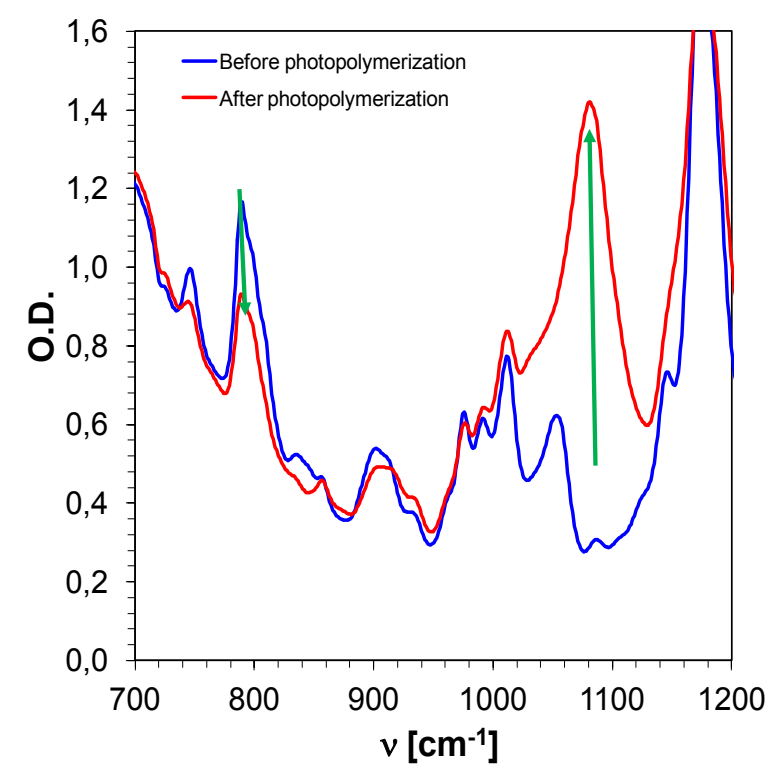

Figure S89. Spectra before and after photopolymerization of epoxy monomer with initiator $\alpha-C N-P I$ under LED $365 \mathrm{~nm}$.

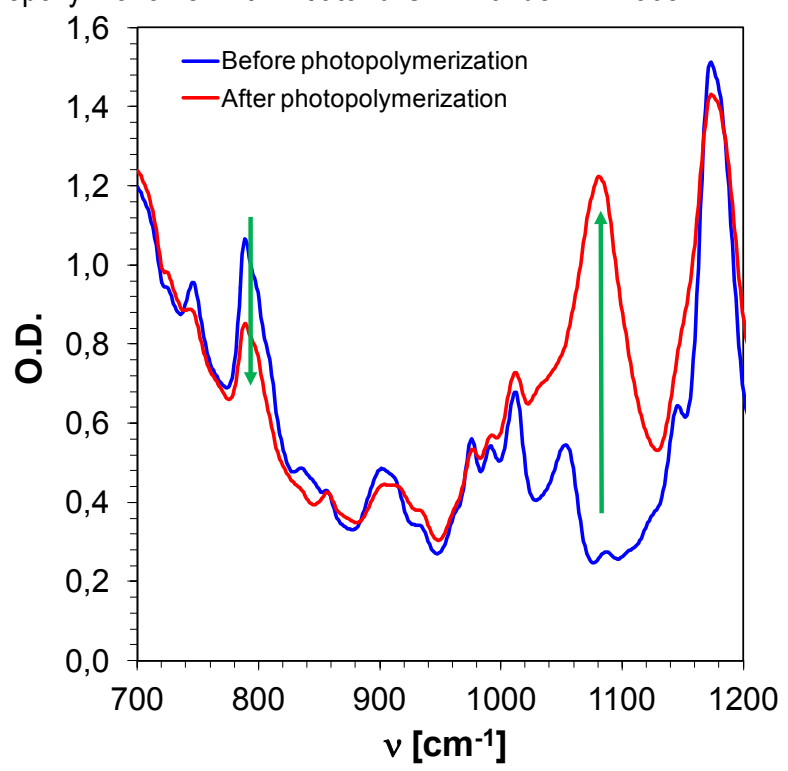

Figure S91. Spectra before and after photopolymerization of epoxy monomer with initiator Ar-2,4-OMe-PI under LED $365 \mathrm{~nm}$.

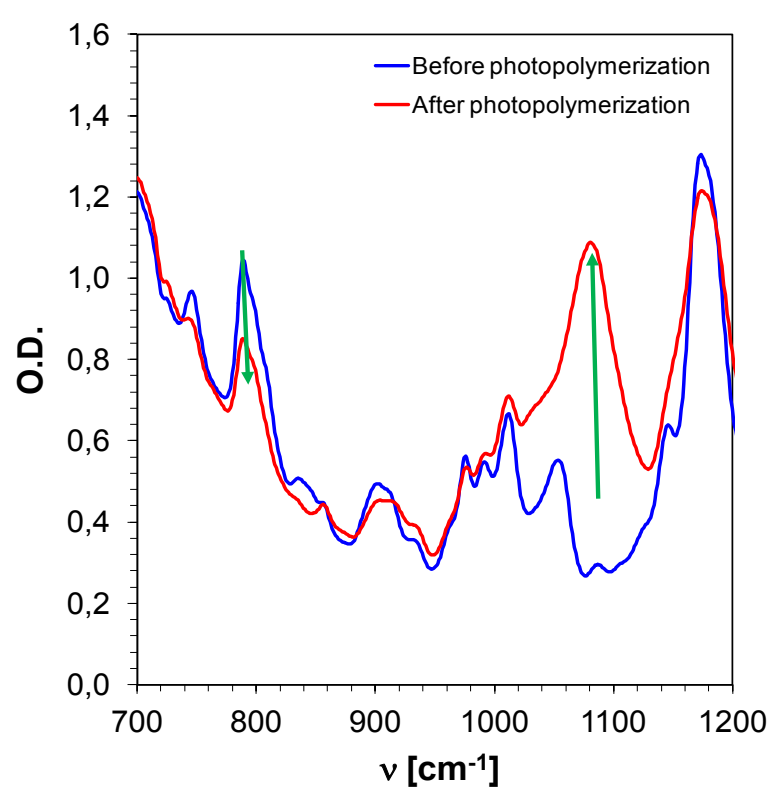

Figure S90. Spectra before and after photopolymerization of epoxy monomer with initiator $\beta$-Ph-PI under LED $365 \mathrm{~nm}$.

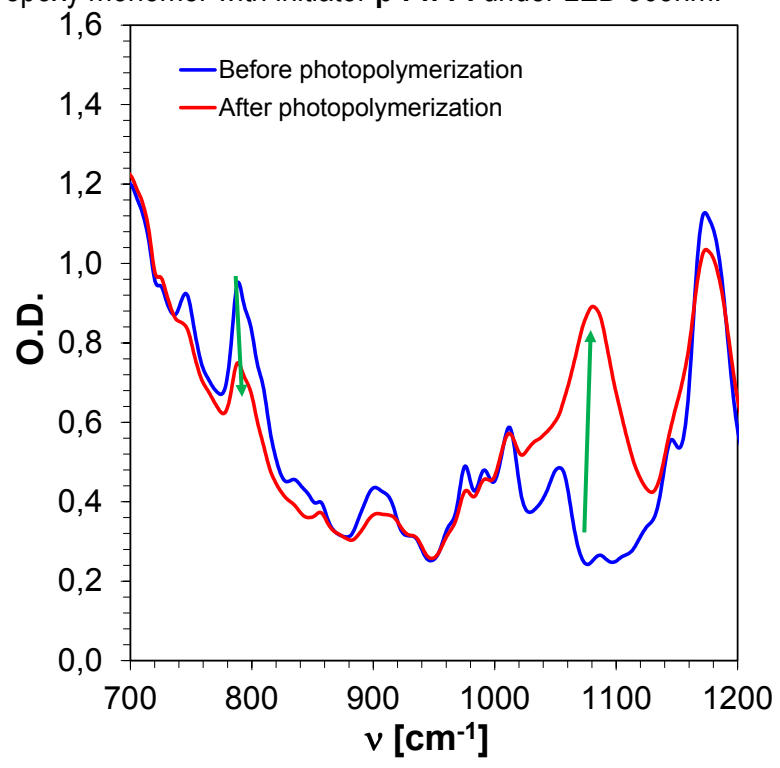

Figure S92. Spectra before and after photopolymerization of epoxy monomer with initiator Comb-Me-PI under LED $365 \mathrm{~nm}$. 


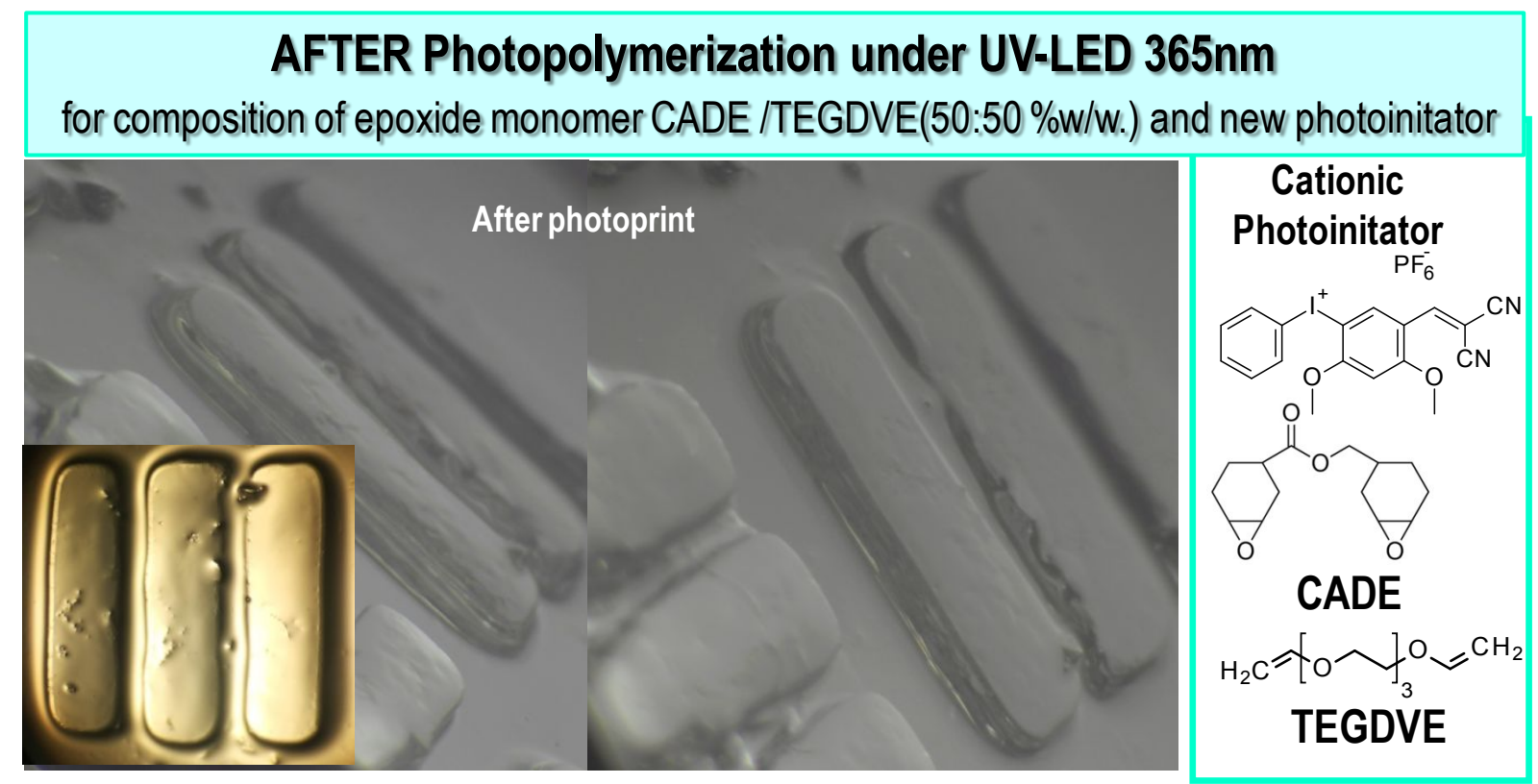

Figure S93. Photographs of samples of 3D prints obtained with new cationic photoinitiator a-CN-PI and CADE/TEGDVE(1:1 wt) composition.

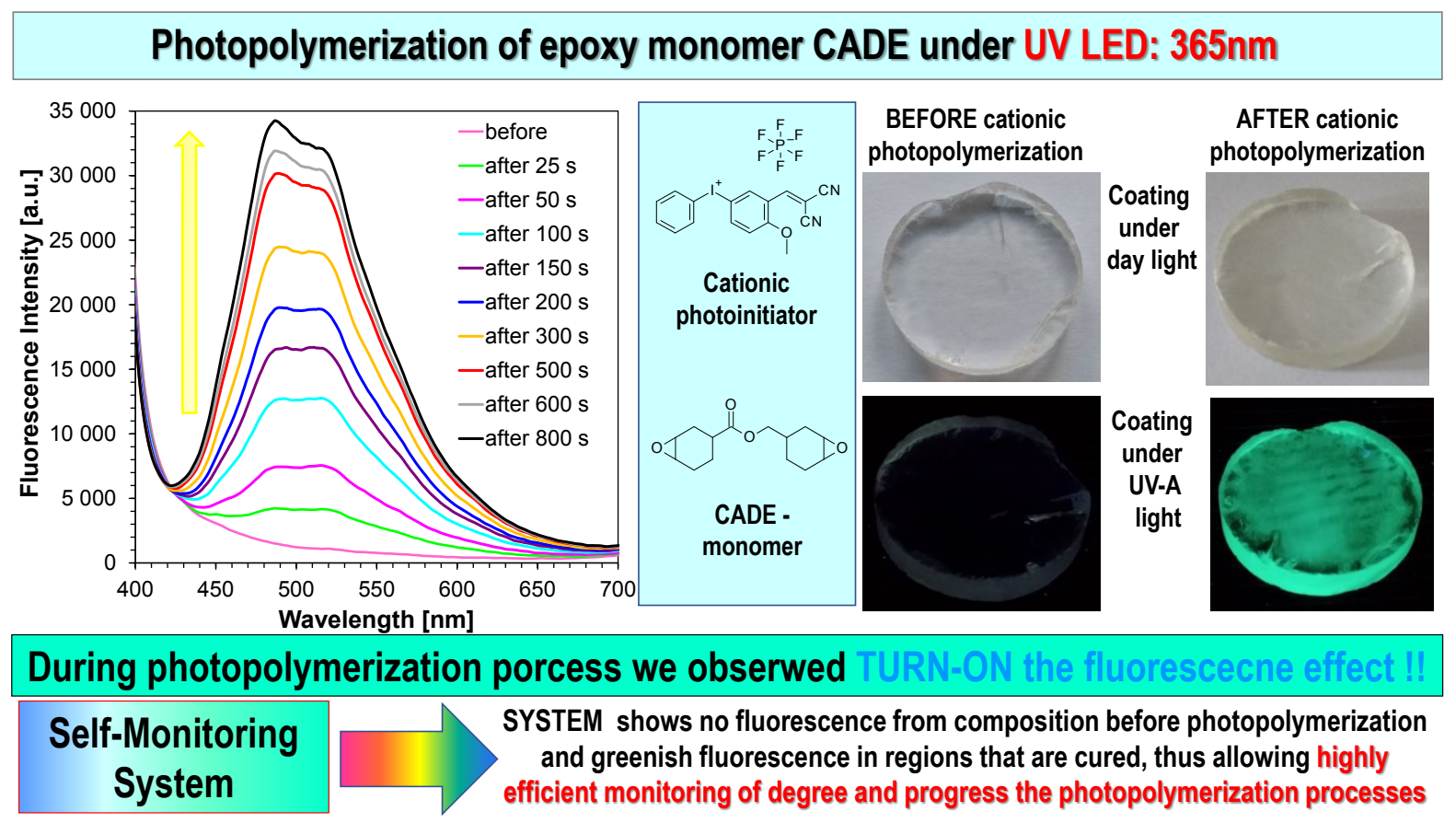

Figure S94. Increase of fluorescence intensity of polymerized coating as a self-monitoring system for photopolymerization process. 
9. In situ monitoring of photopolymerization by photoproduct of photolysis of new iodonium photoinitiators with luminescence characteristics

Photopolymerization of vinyl monomer (TEGDVE)

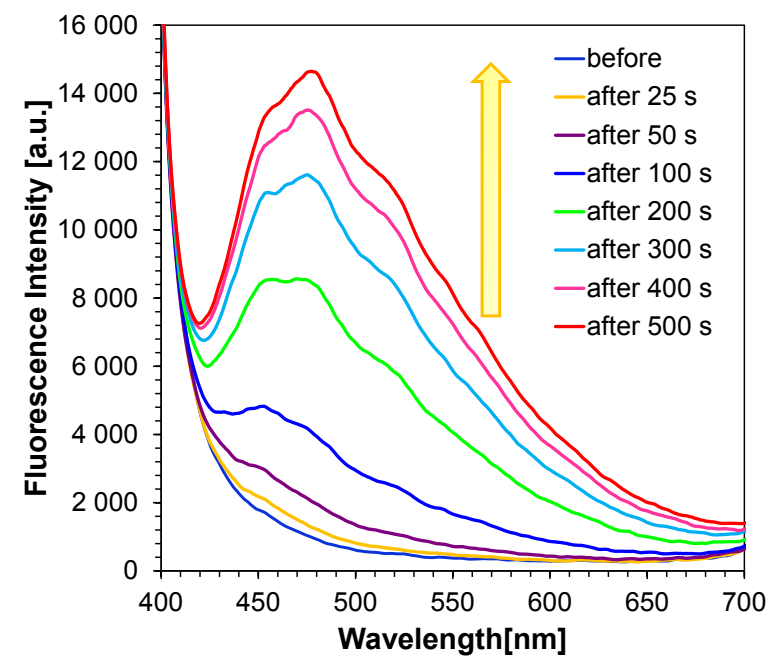

Figure S95. The luminescence spectra of Ar-2,4-OMe-PI before, during and after photopolymerization of vinyl monomer (TEGDVE).

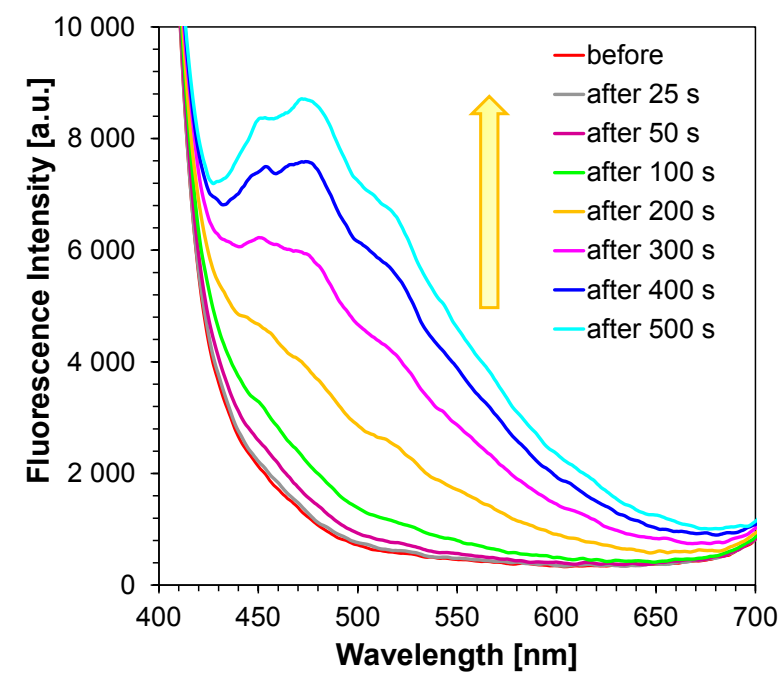

Figure S97. The luminescence spectra of Ar-2,4,6-OMePI before, during and after photopolymerization of vinyl monomer (TEGDVE).

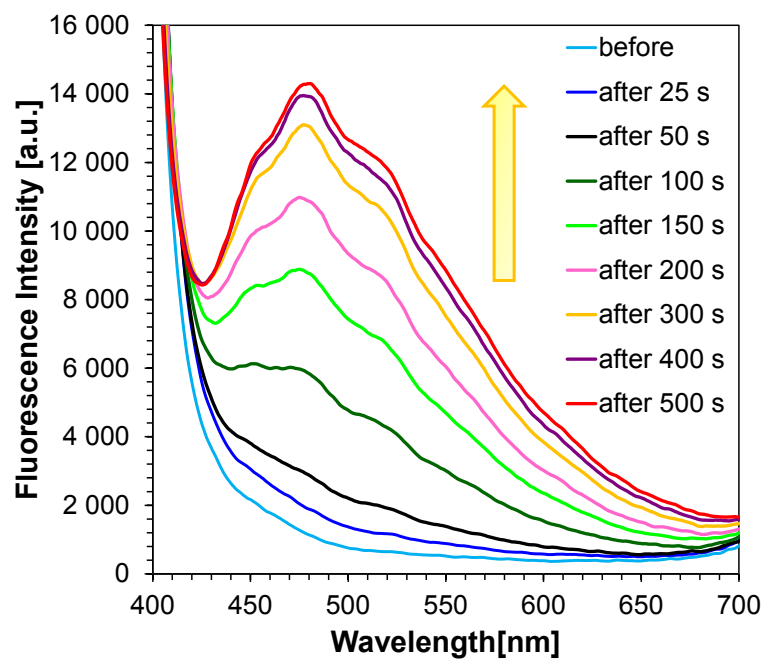

Figure S96. The luminescence spectra of Ar-2,6-OMe-PI before, during and after photopolymerization of vinyl monomer (TEGDVE).

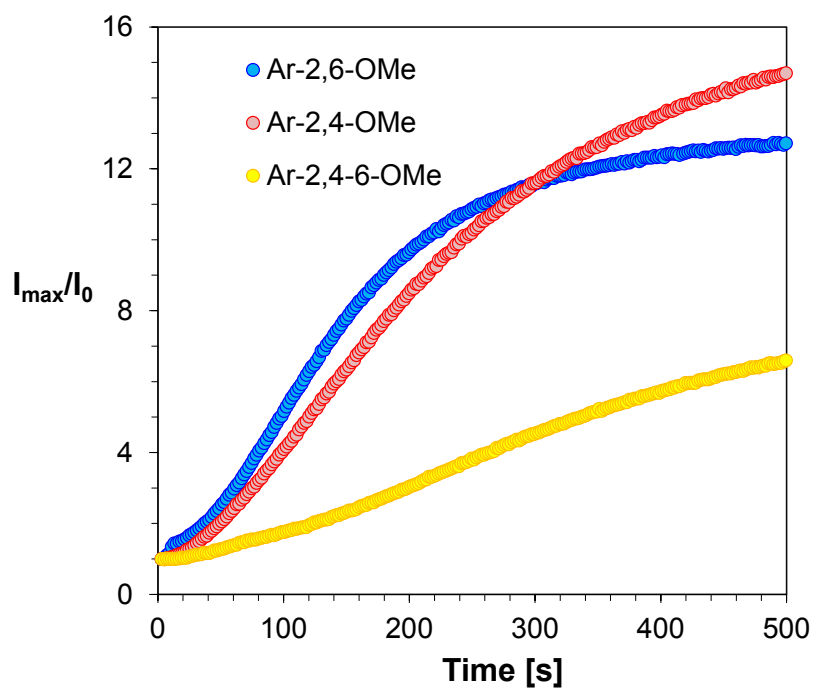

Figure S98. Changes of relative luminescence intensity $\left(I_{\max } / I_{0}\right)$ of photoinitiators during photopolymerization of vinyl monomer (TEGDVE). 


\section{Bibliography:}

1. H. Xu, L. Pan, X. Fang, B. Liu, W. Zhang, M. Lu, Y. Xu, T. Ding and H. Chang, Tetrahedron Lett., 2017, 58, $2360-2365$

2. F. Tamaddon and D. Azadi, J. Iran. Chem. Soc., 2017, 14, 2077 - 2086

3. K. Y. Chu, J. Griffiths and D. Ward, J. Chem. Res., 1981, 10, $3701-3721$

4. A. T. Shulgin, J. Med. Chem., 1966, 9, $445-446$

5. J. C. Sircar, C. R. Kostlan, R. B. Gilbertsen, M. K. Bennett, M. K. Dong and W. J. Cetenko, J. Med. Chem., 1992, 35, $1605-1609$

6. O. O. E. Hormi, C. Peltonen and R. Bergstroem, J. Chem. Soc. Perkin Trans. 1, 1991, 1, 219 - 222

7. CIBA, FR1517178, 1967

8. M. Campaigne, J. Heterocycl. Chem., 1975, 12, $267-269$

9. X. Sun, M. Jin, X. Wu, H. Pan, D. Wan and H. Pu, J. Polym. Sci. Pol. Chem., 2018, 56, 776-782.

10. G. Pohlers, J. C. Scaiano and R. Sinta, Chem. Mater., 1997, 9, 3222-3230.

11. C. G. Hatchard and C. A. Parker, Proc. R. Soc. Lond. A, 1956, 235, 518-536.

12. H. J. Kuhn, S. E. Braslavsky and R. Schmidt, Pure \& Appl. Chem., 1989, 61, 187-210. 